\title{
An introduction to operator preconditioning for the fast iterative integral equation solution of time-harmonic scattering problems
}

\author{
Xavier ANTOINE · Marion DARBAS
}

Received: date / Accepted: date

\begin{abstract}
The aim of this paper is to provide an introduction to the improved iterative Krylov solution of boundary integral equations for time-harmonic scattering problems arising in acoustics, electromagnetism and elasticity. From the point of view of computational methods, considering large frequencies is a challenging issue in engineering since it leads to solving highly indefinite large scale complex linear systems which generally implies a convergence breakdown of iterative methods. More specifically, we explain the problematic and some partial solutions through analytical preconditioning for high-frequency acoustic scattering problems and the introduction of new combined field integral equations. We complete the paper with some recent extensions to the case of electromagnetic and elastic waves equations.
\end{abstract}

Keywords time-harmonic scattering · acoustic · electromagnetism · elasticity · integral equation · Krylov solver $\cdot$ preconditioners $\cdot$ combined field integral equation

\section{Contents}

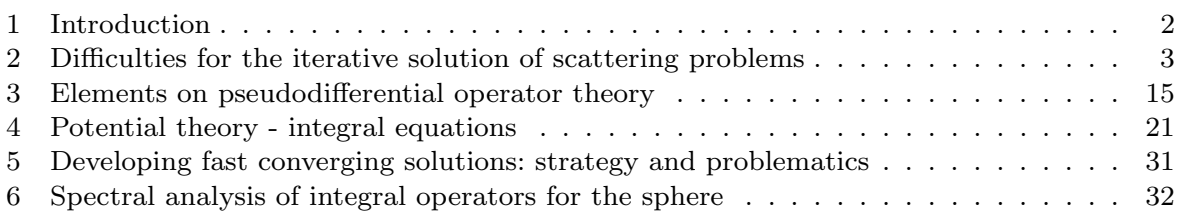

X. Antoine

Université de Lorraine, CNRS, Inria, IECL, F-54000 Nancy, France.

E-mail: xavier.antoine@univ-lorraine.fr

M. Darbas

LAMFA UMR CNRS 7352, Université de Picardie Jules Verne, 33 rue Saint-Leu 80039

Amiens France

E-mail: marion.darbas@u-picardie.fr 


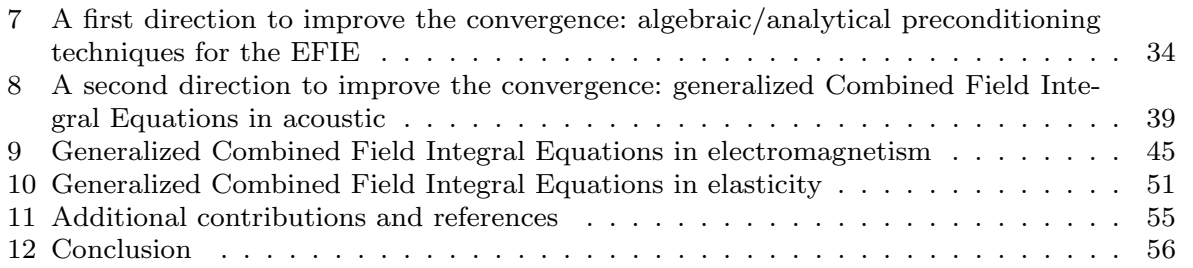

\section{Introduction}

The numerical solution of time-harmonic (exterior) scattering problems in the high frequency regime remains a challenging problem due to its specific computational drawbacks. A few possible directions can be considered to formulate the problem and try to solve it $[19,51,56,81,82,88,99,100,116$. Here, we choose the point of view of integral equations formulations [56, 88, 100, 113. In the most recent industrial code developments, a boundary integral equation formulation is approximated by the Boundary Element Methods (BEM) 82. The resulting linear system is next solved by an iterative Krylov solver [69, 106 coupled e.g. with a Fast Multipole Method (FMM) [51, 55, 62, 63, 70, 82, 105 111 or $\mathcal{H}$-matrix $26,72,84$. However, for example in acoustic, the Helmholtz operator for scattering problems is a highly indefinite complex-valued linear operator. As a consequence, the associated matrix resulting from the boundary element discretization is also highly indefinite and complex. This results in a breakdown of the iterative Krylov solver in many applications or to an extremely slow convergence in best cases. The aim of this paper is to explain the specific difficulties linked to this problem and to introduce some partial solutions to solve the problem. In particular, the convergence of the solver is closely related to the spectral properties of the integral operators. We propose here to modify the eigenvalue distribution in such a way that we get a fast convergence. This is related to the idea of preconditioning but not only. Furthermore, the originality of our approach is that we work at the continuous level, meaning that we directly manipulate the Helmholtz operator and not its discrete matrix representation. This point of view gives rise to an analytical operator theory for building preconditioners which must be compared to the standard purely algebraic approach.

The plan of the paper is the following. In Section 2, we give some basics about numerical linear algebra and Krylov solvers. In particular, we describe on a few explicit examples the problems of convergence that can arise when solving indefinite linear systems. This leads us to analyze and understand why high frequency exterior Helmholtz problems also suffer from a lack of convergence. A brief review of some notions and recent developments on algebraic preconditioners is also proposed for convergence improvement. As already said, one of the originalities of our approach for preconditioning integral operators is to build continuous analytical preconditioners. To this aim, Section 3 gives an introduction to the theory of pseudodifferential operators and associated symbolic calculus which will be used in the sequel. In particular, we give a 
first simple application of this theory for proposing an original interpretation of standard algebraic Laplace shifted preconditioners. Section 4 focuses on the theory of potential which is used for writing integral equation representations for acoustic scattering. We develop the elementary notions for obtaining integral equations and review standard direct and indirect, first- and second-kind Fredholm integral operator formulations. This allows us to insist on the spectral properties of each representation and to precise the well-posedness results. Section 5 gives a short presentation of the way actual integral equation solvers are built. This mainly highlights the fact that the construction of a preconditioner should be based on the only assumption that an integral operator is never available through its matrix but rather through a "black-box" which is able to compute fast matrix-vector products. Therefore, a suitable preconditioner should be matrix-free which is a strong restriction to usual algebraic preconditioners. We analyze in Section 6 the spectral distribution of standard integral operators in the case of a sphere where integral operators can be diagonalized. This gives a thorough understanding of the eigenvalue distribution of each operator and how it is related to the frequency parameter $k$ and density $n_{\lambda}$ of discretization points per wavelength. In Section 7, we describe two possible solutions for obtaining some efficient and robust analytical preconditioners. The first strategy uses integral operators through the so-called Calderón relations while the second one is based on the theory of pseudodifferential operators. Some examples show that these two directions are promising. In Section 8, we propose to build some new alternative well-posed integral equations with a spectral distribution well-suited to get a fast convergence of an iterative solver. Examples show that these new integral formulations are well-adapted for solving high frequency acoustic problems. In Sections 9 and 10, we discuss the extension of this approach to the Maxwell's equations and elasticity equations, respectively. In Section 11, we briefly review other recent contributions for completeness. Finally, Section 12 gives a conclusion.

\section{Difficulties for the iterative solution of scattering problems}

\subsection{Notations and background}

Let us begin by some background in linear algebra. A vector norm on a vector space $\mathbb{Y}$ is a real-valued function $\mathbf{y} \mapsto\|\mathbf{y}\|$ on $\mathbb{Y}$ which satisfies

- $\|\mathbf{y}\| \geq 0, \forall \mathbf{y} \in \mathbb{Y}$, and $\|\mathbf{y}\|=0$ if and only if $\mathbf{y}=\mathbf{0}$ (positivity),

- $\|\alpha \mathbf{y}\|=|\alpha|\|\mathbf{y}\|, \forall \mathbf{y} \in \mathbb{Y}, \forall \alpha \in \mathbb{C}$ (scaling),

- $\|\mathbf{y}+\mathbf{x}\| \leq\|\mathbf{y}\|+\|\mathbf{x}\|, \forall \mathbf{y}, \mathbf{x} \in \mathbb{Y}$ (triangular inequality).

For the particular case when $\mathbb{Y}=\mathbb{C}^{n}$, the most commonly used vector norms are the Hölder norms ( $p$-norms)

$$
\|\mathbf{y}\|_{p}=\left(\sum_{i=1}^{n}\left|y_{i}\right|^{p}\right)^{1 / p}
$$


with $\mathbf{y}=\left(y_{i}\right)_{i=1, \cdots, n}$ and $p \geq 1$. The most useful norms in practice are

$$
\|\mathbf{y}\|_{1}=\sum_{i=1}^{n}\left|y_{i}\right|,\|\mathbf{y}\|_{2}=\left(\sum_{i=1}^{n}\left|y_{i}\right|^{2}\right)^{1 / 2}, \text { and }\|\mathbf{y}\|_{\infty}=\max _{i=1, \cdots, n}\left|y_{i}\right| .
$$

We can use norms to measure the magnitude of a matrix. The same axioms stated above for vector norms apply here

- $\|A\| \geq 0, \forall A \in \mathbb{C}^{n \times n}$, and $\|A\|=0$ if and only if $A=0$ (positivity),

- $\|\alpha A\|=|\alpha|\|A\|, \forall A \in \mathbb{C}^{n \times n}, \forall \alpha \in \mathbb{C}$ (scaling),

- $\|A+B\| \leq\|A\|+\|B\|, \forall A, B \in \mathbb{C}^{n \times n}$ (triangular inequality).

Important norms are the induced matrix $p$-norms

$$
\|A\|_{p}=\max _{\mathbf{x} \in \mathbb{C}^{n}, \mathbf{x} \neq 0} \frac{\|A \mathbf{x}\|_{p}}{\|\mathbf{x}\|_{p}} .
$$

A fundamental property is that

$$
\|A B\|_{p} \leq\|A\|_{p}\|B\|_{p}, \forall A, B \in \mathbb{C}^{n \times n} .
$$

When $p=1$ and $p=\infty$, we have the simple formulas $\left(A=\left(a_{i j}\right)_{1 \leq i, j \leq n}\right)$

$$
\|A\|_{1}=\max _{j=1, \cdots, n} \sum_{i=1}^{n}\left|a_{i j}\right| \text { and }\|A\|_{\infty}=\max _{i=1, \cdots, n} \sum_{j=1}^{n}\left|a_{i j}\right| .
$$

The induced 2-norm is also called the spectral norm and is given by

$$
\|A\|_{2}=\rho\left(A^{*} A\right)^{1 / 2},
$$

with $A^{*}$ the transpose conjugate matrix $\left(=\bar{A}^{T}\right)$ and $\rho(A)$ the maximum modulus of the eigenvalues of $A$ (spectral radius).

To end this section, let us give some other useful definitions.

Definition 1 Let us introduce the following definitions

- A non Hermitian matrix $A$ of size $n \times n$ is said to be positive-definite (respectively negative-definite) if and only if $\Re\left(\mathbf{x}^{*} A \mathbf{x}\right)>0$ (respectively $\left.\Re\left(\mathbf{x}^{*} A \mathbf{x}\right)<0\right)$ for all non-zero vectors $\mathbf{x} \in \mathbb{C}^{n}$.

- A Hermitian matrix $A\left(A=A^{*}\right)$ of size $n \times n$ is called positive-definite (respectively negative-definite) if and only if $\mathbf{x}^{*} A \mathbf{x}>0$ (respectively $\mathbf{x}^{*} A \mathbf{x}<$ $0)$ for all non-zero vectors $\mathbf{x} \in \mathbb{C}^{n}$.

- A Hermitian matrix $A$ of size $n \times n$ is said to be positive-semidefinite (respectively negative-semidefinite) if and only if $\mathbf{x}^{*} A \mathbf{x} \geq 0$ (respectively $\left.\mathrm{x}^{*} A \mathrm{x} \leq 0\right)$ for all $\mathrm{x} \in \mathbb{C}^{n}$.

- A Hermitian matrix which is neither positive- or negative-semidefinite is called indefinite.

We also have the following Proposition.

Proposition 1 A Hermitian matrix is positive-definite if and only if all its eigenvalues are real and strictly positive. 
2.2 Iterative algorithms

We consider a linear system

$$
A \mathbf{x}=\mathbf{b} \text {, }
$$

where $A$ is an invertible complex-valued matrix of size $n \times n$ and $\mathbf{b} \in \mathbb{C}^{n}$. Approaches for the solution of linear systems fall into two classes: direct methods and iterative methods.

Direct methods [106] produce an exact solution in a predictable finite number of elementary operations assuming no rounding errors. When the matrix is symmetric positive-definite, a Cholesky algorithm is applied. For a nonsymmetric matrix, a gaussian elimination solver is used. In this case, the memory storage and computational times costs scale as $\mathcal{O}\left(n^{2}\right)$ and $\mathcal{O}\left(n^{3}\right)$ respectively. Then, a direct solution is clearly out of reach when $A$ is large and dense, requiring hence too large memory and prohibitive computational times.

Iterative schemes can be considered as an alternative to direct methods for the solution of large linear systems. Stationary relaxation-type methods (Jacobi, Gauss-Seidel...) [69,106] have the disadvantage of slow convergence and concern certain classes of matrices only. Projection methods are more general and robust. This is most particularly the case of Krylov subspace methods [69 106]. These techniques are motivated by the Cayley-Hamilton theorem 91] which allows to construct the inverse of a matrix as a polynomial according to $A$. Krylov subspace methods consist in seeking an approximate solution $\mathbf{x}^{(m)}$ to 11 from an affine subspace

$$
\mathbf{x}^{(0)}+\mathcal{K}_{m}\left(A, \mathbf{r}^{(0)}\right)
$$

with

- $\mathbf{x}^{(0)}$ the initial guess,

- $\mathbf{r}^{(0)}=\mathbf{b}-A \mathbf{x}^{(0)}$ the initial residual vector,

- $\mathcal{K}_{m}\left(A, \mathbf{r}^{(0)}\right)=\operatorname{span}\left\{\mathbf{r}^{(0)}, A \mathbf{r}^{(0)}, A^{2} \mathbf{r}^{(0)}, \ldots, A^{m-1} \mathbf{r}^{(0)}\right\}$ the Krylov subspace of dimension $m$,

such that the orthogonality condition (Petrov-Galerkin condition)

$$
\left(B\left(\mathbf{x}-\mathbf{x}^{(m)}\right), \mathbf{v}\right)=0, \quad \forall \mathbf{v} \in \mathcal{K}_{m}\left(A, \mathbf{r}^{(0)}\right),
$$

is fulfilled. The successive approximations are clearly expressed by $\mathbf{x}^{(m)}=$ $\mathbf{x}^{(0)}+P_{m-1}(A) \mathbf{r}^{(0)}$, where $P_{m-1}$ is a polynomial of degree $m-1$. The different Krylov methods therefore differ in the choice of the matrix $B$ (generally symmetric positive-definite). If $B$ defines a scalar product, then the orthogonality condition (3) becomes equivalent to the following minimization problem (optimality condition)

$$
\left\|\mathbf{x}-\mathbf{x}^{(m)}\right\|_{B}=\min _{\mathbf{y} \in \mathcal{K}_{m}\left(A, \mathbf{r}^{(0)}\right)}\|\mathbf{x}-\mathbf{y}\|_{B} .
$$

Two standard examples of such methods are 
- Conjugate gradient methods: If $A$ is symmetric positive-definite, then $B=$ $A$ and (3) is equivalent to

$$
\left\|\mathbf{x}-\mathbf{x}^{(m)}\right\|_{A}=\min _{\mathbf{y} \in \mathcal{K}_{m}\left(A, \mathbf{r}^{(0)}\right)}\|\mathbf{x}-\mathbf{y}\|_{A},
$$

- Generalized Minimum RESidual method (GMRES) : For a general matrix $A$, we choose $B=A^{T} A$ and get

$$
\left\|\mathbf{b}-A \mathbf{x}^{(m)}\right\|_{2}=\min _{\mathbf{x} \in \mathcal{K}_{m}\left(A, \mathbf{r}^{(0)}\right)}\|\mathbf{b}-A \mathbf{x}\|_{2} .
$$

A Krylov subspace method is then represented by a constructive algorithm of a basis of the affine subspace $\mathcal{K}_{m}\left(A, \mathbf{r}^{(0)}\right)$ and by an optimality criterion to determine the approximate solution $\mathbf{x}^{(m)}$ of 1 .

Let us now focus our attention on the GMRES which constitutes the reference algorithm for integral equations. This algorithm, introduced by Saad and Schultze 107, is well-adapted to solve large nonsymmetric linear systems. It corresponds to the choice of the Krylov subspace $\mathcal{K}_{m}\left(A, \mathbf{v}_{1}\right)$, with $\mathbf{v}_{1}=\mathbf{r}^{(0)} /\left\|\mathbf{r}^{(0)}\right\|_{2}$. At each iteration, $\mathcal{K}_{m}\left(A, \mathbf{v}_{1}\right)$ has to be constructed. In practice, we have to generate a set of basis of this subspace. The natural basis $\left(\mathbf{v}_{1}, A \mathbf{v}_{1}, A^{2} \mathbf{v}_{1}, \ldots, A^{m-1} \mathbf{v}_{1}\right)$ cannot be used because of its numerical degeneracy. A solution is to construct an orthonormal basis $\left(\mathbf{v}_{1}, \mathbf{v}_{2}, \ldots, \mathbf{v}_{m}\right)$ of $\mathcal{K}_{m}\left(A, \mathbf{v}_{1}\right)$ via the Arnoldi-Modified Gramm-Schmidt algorithm. We denote by $V_{m}=\left(\mathbf{v}_{1}, \mathbf{v}_{2}, \ldots, \mathbf{v}_{m}\right)$ the $n \times m$ matrix with columns vectors $\mathbf{v}_{i}, i=1, \ldots, n$, and by $\bar{H}_{m}$ the $(m+1) \times m$ Hessenberg matrix where nonzero entries $h_{i j}=$ $\left(A \mathbf{v}_{j}, \mathbf{v}_{i}\right)$ are defined by the Arnoldi-Modified Gramm-Schmidt algorithm. Any vector $\mathbf{x}$ in $\mathbf{x}^{(0)}+\mathcal{K}_{m}\left(A, \mathbf{v}_{1}\right)$ can thus be written as $\mathbf{x}=\mathbf{x}^{(0)}+V_{m} \mathbf{y}$, where $\mathbf{y} \in \mathbb{C}^{m}$. Moreover, the relation $A V_{m}=V_{m+1} \bar{H}_{m}$ holds. It results in

$$
\mathbf{b}-A \mathbf{x}=\mathbf{b}-A\left(\mathbf{x}^{(0)}+V_{m} \mathbf{y}\right)=V_{m+1}\left(\beta \mathbf{e}_{1}-\bar{H}_{m} \mathbf{y}\right),
$$

by setting $\beta=\left\|\mathbf{r}^{(0)}\right\|_{2}$ and $\mathbf{e}_{1}=(1,0, \ldots, 0)^{T} \in \mathbb{R}^{m+1}$. Then, we have the optimality criterion

$$
\min _{\mathbf{x} \in \mathcal{K}_{m}\left(A, \mathbf{r}^{(0)}\right)}\|\mathbf{b}-A \mathbf{x}\|_{2}=\min _{\mathbf{y} \in \mathbb{R}^{m}}\left\|\beta \mathbf{e}_{1}-\bar{H}_{m} \mathbf{y}\right\|_{2},
$$

exploiting the fact that the column-vectors of $V_{m+1}$ are orthonormal. The approximate solution $\mathbf{x}^{(m)}$ can be obtained as $\mathbf{x}^{(m)}=\mathbf{x}^{(0)}+V_{m} \mathbf{y}^{(m)}$, where $\mathbf{y}^{(m)}$ minimizes the functional $J(\mathbf{y})=\left\|\beta \mathbf{e}_{1}-\bar{H}_{m} \mathbf{y}\right\|_{2}$. The pseudocode for a basic form of the GMRES algorithm can now be given as

\section{Initialization}

Compute $\mathbf{r}^{(0)}=\mathbf{b}-A \mathbf{x}^{(0)}, \beta:=\left\|\mathbf{r}^{(0)}\right\|_{2}$ and $\mathbf{v}_{1}:=\mathbf{r}^{(0)} / \beta$

2. Define the $(m+1) \times m$ matrix $\bar{H}_{m}=\left\{h_{i j}\right\}_{1 \leq i \leq m+1,1 \leq j \leq m}$. Set $\bar{H}_{m}=0$.

3. Construction of the Arnoldi's basis (steps 3-11)

for $j=1,2, \ldots, m$, do

4. compute $\mathbf{w}_{j}:=A \mathbf{v}_{j}$

5. for $i=1, \ldots, j$, do 
6. $\quad h_{i j}:=\left(\mathbf{w}_{j}, \mathbf{v}_{i}\right)$

7. $\quad \mathbf{w}_{j}:=\mathbf{w}_{j}-h_{i j} \mathbf{v}_{i}$

8. $\quad$ enddo

9. $h_{j+1, j}=\left\|\mathbf{w}_{j}\right\|_{2}$. If $h_{j+1, j}=0$ set $m:=j$ and go to 12

10. $\mathbf{v}_{j+1}=\mathbf{w}_{j} / h_{j+1, j}$

11. enddo

12. Minimization problem

Compute $\mathbf{y}^{(m)}$ as the minimizer of $\left\|\beta \mathbf{e}_{1}-\bar{H}_{m} \mathbf{y}\right\|_{2}$ and $\mathbf{x}^{(m)}=\mathbf{x}^{(0)}+$ $V_{m} \mathbf{y}^{(m)}$.

In order to solve the least-squares problem (step 12), the most adapted technique is to employ a QR-decomposition of the Hessenberg matrix $\bar{H}_{m}$ (see [106] for details).

Remark 1 The parameter $m$ is not determined a priori. In general, a maximum number $m_{\max }$ is fixed and is typically dictated by the computational ressources. If the maximum number $m_{\max }$ of iterations has been reached without triggering the convergence test, then a restarting is done, i.e. GMRES is started afresh with the last approximation $\mathbf{x}^{\left(m_{\max }\right)}$ as the initial guess. This method is called restarted GMRES $\left(m_{\text {max }}\right)$. The residual $\left\|\mathbf{b}-A \mathbf{x}^{(m)}\right\|_{2}$ is generally used as a stopping criterion.

Essentially, the computational cost of GMRES is related to

i) the total number of iterations $N^{\text {iter }}$ required to reach an a priori fixed tolerance $\varepsilon$ on the residual norm,

ii) the cost of one iteration which is mainly the Matrix-Vector Product (MVP) $\mathbf{w}_{j}=A \mathbf{v}_{j}$ involved at step 4 .

If this algorithm is directly used for a full complex-valued matrix $A$, then the total cost is $\mathcal{O}\left(N^{\text {iter }} n^{2}\right)$. In terms of memory storage, the algorithm still needs $\mathcal{O}\left(n^{2}\right)$ entries for $A$. In the background of integral equations, efficient compression algorithms have been proposed during the last decades. For example, the multilevel FMM [51,55, 62, 63, 70, 82, 105, 111 both computes the MVP in $\mathcal{O}(n \log n)$ operations and requires $\mathcal{O}(n)$ entries. Essentially, the FMM proposes to compute only the near-field entries exactly and the far-field entries approximately (but with controllable error). Other fast algorithms exists like e.g. $\mathcal{H}$-matrix compression techniques [26, 72, 84] or high-order solvers developed in 33, 34, 36. This drastic reduction both in computational cost and memory storage for one iteration of the GMRES gives expectations for solving high frequency problems.

Let us now briefly review the most important results on the convergence behaviour of GMRES (for proof and further details see [69, 96, 106]). If $A \in$ $\mathbb{C}^{n \times n}$ is non singular, we define the quantity

$$
\kappa_{p}(A)=\|A\|_{p}\left\|A^{-1}\right\|_{p},
$$

which is called the condition number of the linear system (1) with respect to the induced matrix $p$-norm (cf. section 2.1). We begin by giving a global convergence result. 
Theorem 1 Let $A \in \mathbb{C}^{n \times n}$ be a non singular matrix. The full GMRES algorithm is guaranteed to converge in at most $n$ iterations.

This is true in the absence of round-off errors. Unfortunately, computer arithmetic is not exact. Moreover, this is impractical for large linear systems which indeed require too much iterative steps to reach the convergence. In these situations, a preconditioner can be used to further reduce the number of iterations (see e.g. Section 2.5). In order to predict the behaviour of GMRES, the convergence analysis is concerned with the derivation of upper bounds on the residual norms.

Proposition 2 Let $A \in \mathbb{C}^{n \times n}$ be a non singular matrix and $\mathbf{x}^{(0)} \in \mathbb{C}^{n}$ an initial guess. If we can diagonalize $A$ in the form $A=U \Lambda U^{-1}$, where $\Lambda$ is the diagonal matrix of eigenvalues $\left(\lambda_{i}\right)_{i}$ of $A$ corresponding to the appropriate eigenvectors in $U$, then a bound on the residual norm at iteration $m$ is

$$
\left\|\mathbf{r}^{(m)}\right\|_{2} \leq \kappa_{2}(U) \min _{q \in \mathbb{P}_{m}, q(0)=1} \max _{i=1, \ldots, n} q\left(\lambda_{i}\right)\left\|\mathbf{r}^{(0)}\right\|_{2},
$$

with $\kappa_{2}(U)=\|U\|_{2}\left\|U^{-1}\right\|_{2}$ the condition number of $U$ in the 2-norm.

This bound was the first convergence result for GMRES 107. However, even if $A$ is normal $\left(\kappa_{2}(U)=1\right)$, the bound (4) may fail to provide any reasonable information about the rate of reduction of the GMRES residual norms. For diagonalizable but non normal $A$ ( $U$ far from unitary), $\kappa_{2}(U)$ might be very large, and the bound in (4) might be a large overestimate of the residual norm. Moreover, it is not clear that only the conditioning of the eigenvectors of $A$ should influence the convergence behaviour of GMRES (see Section 2.3. Obtaining computable bounds on the residual norm that generate a good prescribed convergence curve for a general matrix is a difficult task. Theoretical results (min-max approximations on matrix eigenvalues) in this field are still partially known. We see in the sequel of the paper that the analysis of the distribution of the eigenvalues in the complex plane gives a very useful approach for predicting the convergence of the GMRES. Concerning the restarted GMRES, the following proposition holds.

Theorem 2 If $A$ is symmetric positive-definite, then GMRES(m) converges at any $m \geq 1$.

However, the restarted GMRES algorithm can stagnate when the matrix is not definite positive. We will observe this difficulty in the following section.

\subsection{Convergence problems for indefinite linear systems}

In iterative methods, a common belief is that the condition number $\kappa_{2}(A)$ (in the 2-norm) of $A$ is a good measure of the convergence rate of the algorithm. This is generally not true for a complex-valued matrix $A$ where indeed the distribution of the eigenvalues in the complex plane is the most crucial point to 


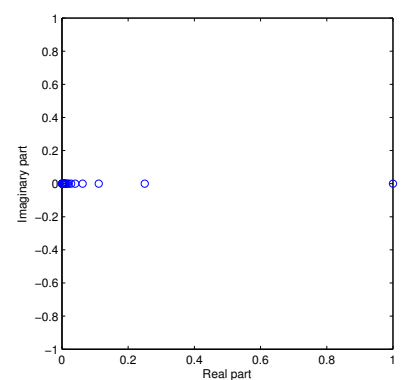

(a) $A_{1}$ : Eigenvalue distribution $(n=100)$

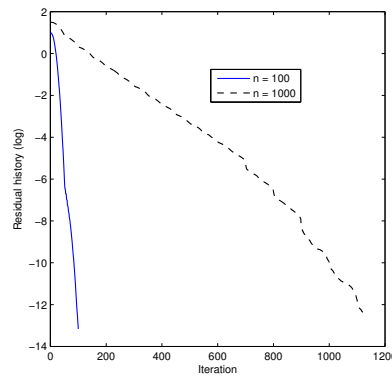

(b) $A_{1}$ : Convergence history

Fig. 1 Matrix $A_{1}$.

observe. In many applications, the eigenvalues of the matrix $A$ are computed in order to examine if the whole spectrum is included or not in a given part of the complex plane. Unfortunately, the matrix is often defined with a given precision and the computed eigenvalues may differ from the real ones, especially in highly non normal cases. To answer to this problem, the difficult notion of $\epsilon$-pseudospectrum of a matrix was introduced [114. In a few words, the idea is to compute the set of the eigenvalues of perturbed matrices $A+E$ for some $E$, with $\|E\| \leq \epsilon$. Generally, this approach is costly in terms of computation.

To illustrate the fact that the distribution of the eigenvalues plays a crucial role in the convergence of the restarted GMRES, let us consider the seven simple complex-valued diagonal matrices $A_{j}$ defined by

$$
\begin{aligned}
& A_{1}=\operatorname{diag}\left(1 / \ell^{2}\right)_{1 \leq \ell \leq n}, \quad A_{2}=\operatorname{diag}\left(e^{i \ell} / \ell^{2}\right)_{1 \leq \ell \leq n}, \quad A_{3}=\operatorname{diag}\left(e^{i \ell}\right)_{1 \leq \ell \leq n}, \\
& A_{4}=\operatorname{diag}\left(1+e^{i \ell}\right)_{1 \leq \ell \leq n}, \quad A_{5}=\operatorname{diag}\left(1.5+e^{i \ell}\right)_{1 \leq \ell \leq n}, \\
& A_{6}=\operatorname{diag}\left(1+e^{i \ell} / \ell\right)_{1 \leq \ell \leq n}, \quad A_{7}=\operatorname{diag}\left(1+e^{i \ell} / \ell^{2}\right)_{1 \leq \ell \leq n} .
\end{aligned}
$$

Concerning $A_{1}$, the matrix is real-valued and the eigenvalues tend towards zero as $\ell \rightarrow+\infty$ (Figure 1(a) . In terms of operators (see section 3), it corresponds to the notion of real-valued elliptic positive pseudodifferential operator of order -2 . More or less, the underlying operator is the inverse of the onedimensional Laplacian operator $\left(\partial_{x}^{2}\right)^{-1}$. In particular, the matrix is symmetric and positive-definite. We can observe that there is an eigenvalue clustering around zero. The condition number $\kappa_{2}\left(A_{1}\right)=n^{2}$ becomes large as $n$ grows and so the convergence of GMRES(50) takes more iterations (Figure 1(b)]. However, the convergence is observed. The tolerance of the iterative solver is fixed equal to $\varepsilon=10^{-14}$ in all the examples.

The second test-case is related to a complex-valued matrix $A_{2}$. The case is close to the previous one but the distribution of the eigenvalues in the complex plane is completely different even if the corresponding magnitude tends to zero (Figure 2(a) . The condition number is again $n^{2}$ but we can quickly observe the divergence of GMRES(50) e.g. for $n=100$ (Figure 2(b)) . Here, 


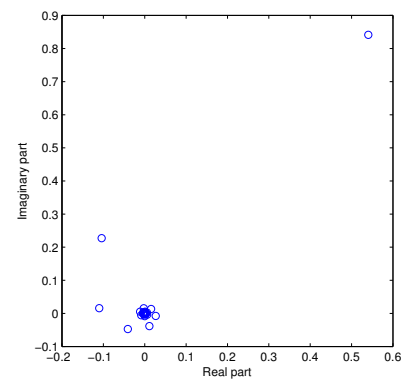

(a) $A_{2}$ : Eigenvalue distribution $(n=100)$

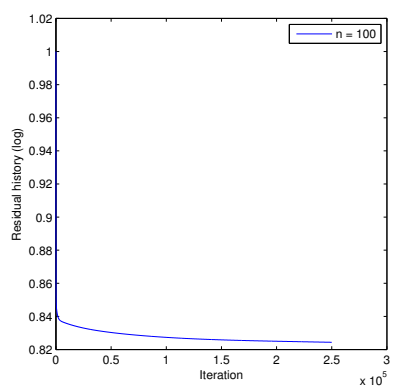

(b) $A_{2}$ : Convergence history

Fig. 2 Matrix $A_{2}$.

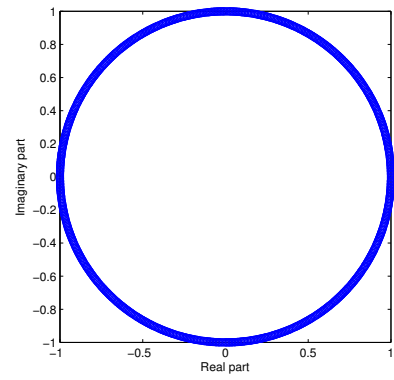

(a) $A_{3}$ : Eigenvalue distribution $(n=1000)$

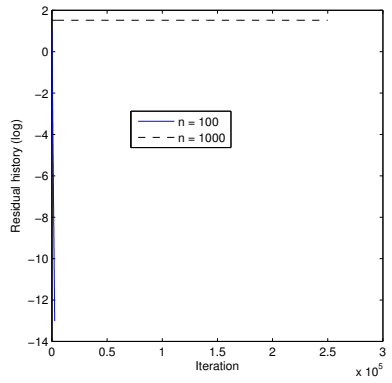

(b) $A_{3}$ : Convergence history

Fig. 3 Matrix $A_{3}$.

the matrix is indefinite (eigenvalues with negative or positive real parts). In terms of operators, this corresponds to an indefinite complex-valued integral (or pseudodifferential) operator of order -2 . This is also a first-kind Fredholm integral operator (see Section 4.3.5). These two situations show how the convergence of the restarted GMRES strongly depends on the distribution of the eigenvalues in the complex plane.

The third situation with $A_{3}$ is also very interesting. The complex-valued matrix has a condition number equals to 1 but the convergence of GMRES(50) takes many iterations to converge for $n=100$ and even diverges for $n=1000$. This example illustrates clearly that considering indefinite matrices leads to instabilities of the restarted GMRES and eventually to its divergence. This is still true if one translates the spectrum of $A_{3}$ from 1 to the right and get the matrix $A_{4}$ (Figure 4(a). Now, if one translates $A_{3}$ from 1.5 and obtain $A_{5}$, then the convergence holds and is relatively fast since all the eigenvalues have a large positive real part and are sufficiently far away from the origin (Figure 4(b). 


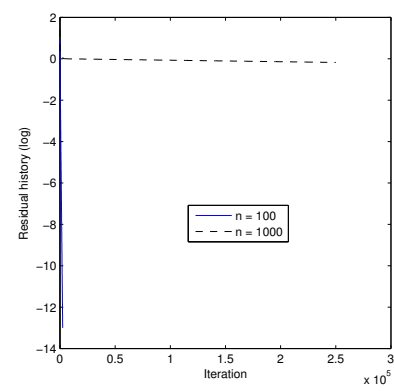

(a) $A_{4}$ : Convergence history

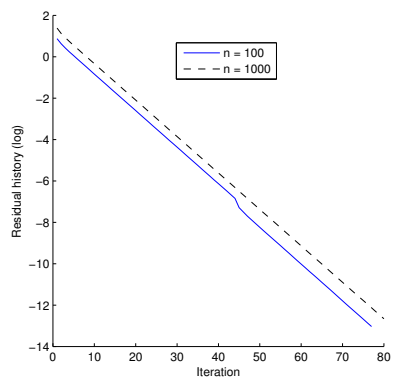

(b) $A_{5}$ : Convergence history

Fig. 4 Matrices $A_{4}$ and $A_{5}$.

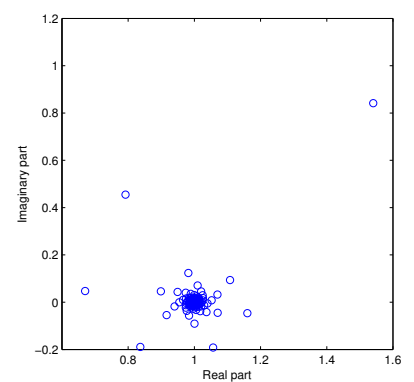

(a) $A_{6}$ : Eigenvalue distribution $(n=100)$

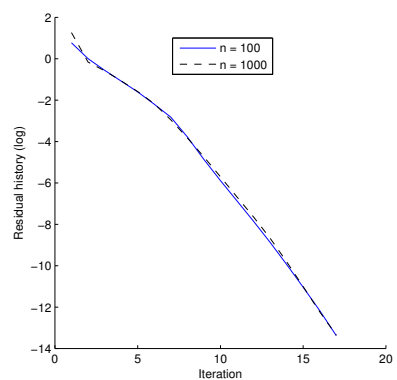

(b) $A_{6}$ : Convergence history $(n=100)$

Fig. 5 Matrix $A_{6}$.

Let us consider the two matrices $A_{6}$ and $A_{7}$. They look like $A_{4}$ but the perturbative term to 1 has a modulus which tends towards zero linearly or quadratically (Figures $5(\mathrm{a})$ and $6(\mathrm{a})$. This rate of convergence of the sequence is related to the order of the underlying operator: first- or second-order. This kind of matrices (close to positive-definite matrices for large values of $n$ ) corresponds to an integral operator called a second-kind Fredholm operator (see Section 4.3.5). Their spectrum clusters around a complex value $\alpha$ (=1 here) up to a sequence converging to zero $\left(e^{i \ell} / \ell\right.$ or $e^{i \ell} / \ell^{2}$ here). These configurations lead to converging iterative schemes with a rate depending on the decay of the sequence to zero (Figures $5(\mathrm{~b})$ and $6(\mathrm{~b})$. 


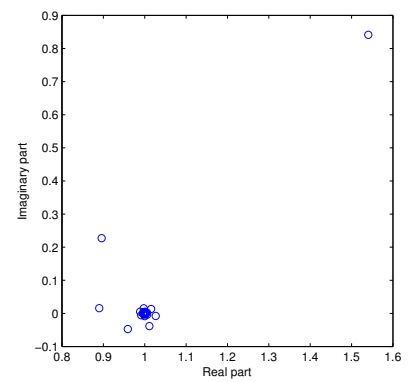

(a) $A_{7}$ : Eigenvalue distribution $(n=100)$

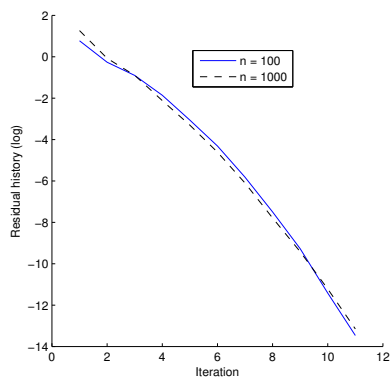

(b) $A_{7}$ : Convergence history

Fig. 6 Matrix $A_{7}$.

2.4 Why this happens in acoustic scattering: a simple example

We have just seen that an indefinite matrix without any robust eigenvalue clustering implies bad convergence properties of the GMRES. Such a situation naturally arises in acoustic scattering.

Let us look at the following simple scattering problem. We consider a plane wave $u^{\text {inc }}(x)=e^{-i k x}$ coming from $+\infty$ and illuminating the left positive domain ] $-\infty ; 0$. The real-valued constant wavenumber is denoted by $k$. The sound-hard scattering problem reads: find the scattered field $u$ in the unbounded domain $[0 ;+\infty[$ such that

$$
\left\{\begin{array}{l}
\left(\partial_{x}^{2}+k^{2}\right) u=0, \quad \text { in }[0 ;+\infty[ \\
\partial_{x} u(0)=i k, \\
u \text { travels to the right. }
\end{array}\right.
$$

Of course, the solution is trivial: $u(x)=e^{i k x}$. Let us note that other boundary conditions could be used as for example the sound-soft one: $u(0)=-1$ or an impedance boundary condition. Since problem (6) is set in an unbounded domain, one usually introduces an Artificial Boundary Condition (ABC) at the fictitious boundary $\Sigma=\{1\}$ (another point could be chosen). Its aim is to replace the sentence " $u$ travels to the right" to get a bounded domain boundary value problem. Here, the boundary condition is trivial and is $\left(\partial_{\boldsymbol{n}}-i k\right) u=0$, where $\boldsymbol{n}$ is the outwardly directed unit normal vector to $\Omega$ at $\Sigma$. Finally, the problem which is solved by a finite element method is

$$
\left\{\begin{array}{l}
\left(\partial_{x}^{2}+k^{2}\right) u=0, \quad \text { in } \Omega, \\
\partial_{x} u(0)=i k, \\
\left(\partial_{\boldsymbol{n}}-i k\right) u=0, \quad \text { on } \Sigma,
\end{array}\right.
$$

with $\Omega=] 0 ; 1[$ and $\Sigma=\{1\}$. Let us consider that we use a linear continuous Galerkin approximation of (7). Then, the variational formulation writes down: 
find $u$ such that

$$
\int_{\Omega} \partial_{x} u \partial_{x} v-k^{2} u v d x-i k u(1) v(1)=-i k v(0)
$$

for all well-chosen test-functions $v$. Let us denote by $\mathbb{M}_{h}$ and $\mathbb{S}_{h}$ respectively the mass and stiffness matrices associated with the linear Finite Element Method (FEM), for a uniform discretization of $\Omega$. The length of one element is $h$ and the total number of degrees of freedom of the FEM is $n_{h}$ (which is equal to the number of segments plus one). We denote by $\mathbf{u}_{h} \in \mathbb{C}^{n_{h}+1}$ the linear approximation of $u$ solution to (7) or (8). The term $-i k u(1) v(1)$ related to the $\mathrm{ABC}$ contributes in the linear system by an additional term on the last row and column of the linear system. We denote this matrix term by $-i k \mathbb{B}_{h}$. The right-hand side is related to $-i k v(0)$ and gives birth to a right-hand side vector $\mathbf{b}_{h} \in \mathbb{C}^{n_{h}+1}$. Finally, the linear finite element approximation of (8) leads to the solution of the system

$$
\left(\mathbb{S}_{h}-k^{2} \mathbb{M}_{h}-i k \mathbb{B}_{h}\right) \mathbf{u}_{h}=\mathbf{b}_{h} .
$$

The sparse matrix involved in system (9) is $i$ ) complex-valued because of the boundary term $-i k \mathbb{B}_{h}$ and $\left.i i\right)$ non positive-definite since we have the contribution $\mathbb{S}_{h}-k^{2} \mathbb{M}_{h}$. This is most particularly penalizing when solving high frequency problems ( $k$ large). We report on Figure $7(\mathrm{a})$ the behaviour of GMRES(50) applied to solving (9) for $k=60$ and $n_{h}=100$. The convergence is extremely slow. Let us note that $n_{h}$ must be quite large for this value of $k$ because of the pollution error [19,113] into the FEM. Figure 7(b) shows the distribution of the eigenvalues of $\left(\mathbb{S}_{h}-k^{2} \mathbb{M}_{h}-i k \mathbb{B}_{h}\right)$ as well as $\left(\mathbb{S}_{h}-k^{2} \mathbb{M}_{h}\right)$. We can clearly see that the problem has many eigenvalues lying in the left halfplane with null imaginary part for $\left(\mathbb{S}_{h}-k^{2} \mathbb{M}_{h}\right)$. As a consequence, the matrix is non positive-definite. Furthermore, the reason why all the eigenvalues are real is that we are rather solving an interior Helmholtz problem with Neumann boundary condition. In the case of an (exterior) scattering problem, adding the $\mathrm{ABC}$ (which means that we consider the additional term $-i k \mathbb{B}_{h}$ ) leads to a complex spectrum. This can be observed on Figure $7(\mathrm{~b})$. Then, this is worst for having a converging iterative scheme.

2.5 How to improve the convergence using preconditioners?

For practical purpose, large scale sparse linear systems are solved by an iterative Krylov solver. This is the case in acoustic scattering where we must be able to design 1) a convergent iterative solver and 2) methods that converge fastly if convergence occurs. One very convenient way to do this is to precondition the linear system by a sparse matrix $P$ called the preconditioner. The idea is the following. Let us assume that we are solving a linear system $A \mathbf{x}=\mathbf{b}$ and that we are able to build a matrix $P$ such that $P$ is close to $A^{-1}$. This means that in some sense, for a suitable matrix norm $\|\cdot\|$, the quantity $\|P A-\mathbb{I}\|$ or $\|A P-\mathbb{I}\|$ is small. Here, $\mathbb{I}$ is the identity matrix. We say that $P$ 


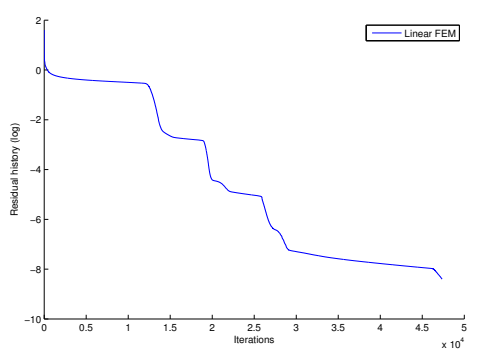

(a) Convergence history

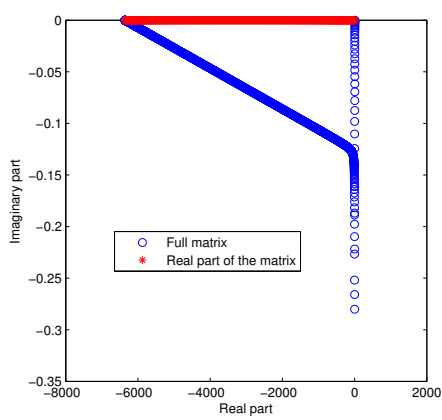

(b) Eigenvalue distribution

Fig. 7 Matrix $\left(\mathbb{S}_{h}-k^{2} \mathbb{M}_{h}-i k \mathbb{B}_{h}\right)$.

is a left preconditioner if $P A \approx \mathbb{I}$ or a right preconditioner if $A P \approx \mathbb{I}$. Since the condition number $\kappa(P A)$ is close to $\kappa(\mathbb{I})=1$, we can guess that the solution to the new linear system $P A \mathbf{x}=P \mathbf{b}$ by an iterative method is convergent and fast. This can be expected for positive-definite matrices $A$ but not necessarily for indefinite matrices as we previously noticed. If applying $P$ only requires a MVP in the GMRES, we say that $P$ is an explicit preconditioner. If its application needs the solution of a sparse linear system, $P$ is said to be implicit.

Since we work at a matrix level, then the construction of the preconditioner $P$ can only be based on algebraic considerations (the entries of $A$ ). Many algebraic preconditioners have been proposed during the last decades. Let us mention e.g. Incomplete LU preconditioners (ILU), SParse Approximate Inverses (SPAI) or Algebraic Recursive Multilevel Solvers (ARMS) and all their variants. We refer to [23] for some of these techniques for general linear systems. Concerning the solution of scattering problems, these preconditioners have been tested and improved for instance in 24, 42, 43, 71, 79, 80, 101, 118. However, even if these preconditioners provide an improved convergence, convergence breakdown and slow convergence still arise when medium and high wavenumbers $k$ are considered within the restarted GMRES. This challenging problem is so still open.

An alternative to the algebraic preconditioners is developed in the sequel. Essentially, we can summarize the derivation of the algebraic preconditioners to: 1) first, take a continuous (partial differential or integrodifferential) operator, 2) discretize it to get a matrix $A$ and 3 ) build an approximate algebraic inverse $P$. Our solution leading to what we call analytical preconditioners is based on the following three points: 1) take a partial differential operator or an integral operator, 2) build an approximate inverse at the continuous level and 3) discretize this operator for example by a finite element method to get a preconditioner $P$. The interesting aspect of this approach is that point 2) keeps the information related to the underlying structure of the operator to 
approximate, information which is a priori lost by considering the algebraic viewpoint. To attain this goal, we need a mathematical theory which allows to compute practically an approximation of the inverse of a general operator at the continuous level. This is the aim of the next section where we introduce the tools from pseudodifferential operators theory and associated microlocal calculus.

\section{Elements on pseudodifferential operator theory}

We introduce in this section the basics of the theory of pseudodifferential operators. The aim of pseudodifferential operators and associated microlocal symbolic calculus is to naturally generalize partial differential and integral operators through the notion of symbol. After the definitions, we give some practical rules for symbolic calculus which are the keystone for building approximations of pseudodifferential operators, with future applications to analytical preconditioning techniques (Sections 3.3, 7, 8, 9 and 10). For further reading, we refer e.g. to 112 where the theory of pseudodifferential operators is presented with more details that cannot be addressed in this short introduction.

3.1 Definitions: pseudodifferential operator and symbol

Let $\Omega \subset \mathbb{R}^{d}$ be an open set and $\mathcal{D}^{\prime}(\Omega)$ the space of distributions on $\Omega$ which is the dual space of $\mathcal{D}(\Omega):=\mathcal{C}_{0}^{\infty}(\Omega)[108,112$. We introduce the vectorial differential operator $D=\left(D_{1}, \cdots, D_{d}\right)$, setting $D_{j}:=-i \partial_{j}=-i \partial_{x_{j}}$. A variable coefficients partial differential operator $P(\boldsymbol{x}, D)$ of order $m$ has the general form

$$
P(\boldsymbol{x}, D)=\sum_{|\alpha| \leq m} a_{\alpha}(\boldsymbol{x}) D^{\alpha},
$$

where $\boldsymbol{x}:=\left(x_{1}, \ldots, x_{d}\right) \in \Omega, \alpha=\left(\alpha_{1}, \cdots, \alpha_{d}\right)$ is a multi-index in $\mathbb{N}^{d}$ and the coefficients $a_{\alpha}$ are $\mathcal{C}^{\infty}(\Omega)$ smooth functions. The operator $D^{\alpha}$ is: $D^{\alpha}=$ $D_{1}^{\alpha_{1}} \ldots D_{d}^{\alpha_{d}}$. For the sake of clarity, we will sometimes precise the derivation variable used for $D^{\alpha}$ like for example $D_{\boldsymbol{x}}^{\alpha}$ or $\partial_{\boldsymbol{\xi}}^{\alpha}$. The polynomial $p: \Omega \times \mathbb{R}^{d} \mapsto \mathbb{C}$

$$
p(\boldsymbol{x}, \boldsymbol{\xi})=\sum_{|\alpha| \leq m} a_{\alpha}(\boldsymbol{x}) \boldsymbol{\xi}^{\alpha}
$$

is called the symbol of the operator $P$ (in the sequel $\boldsymbol{\xi}=\left(\xi_{1}, \ldots, \xi_{d}\right)$ is the Fourier covariable). The principal symbol of order $m$ of $P$, denoted by $\sigma_{p}(P)$, represents the homogeneous part of degree $m$ in $\boldsymbol{\xi}$ of $p$,

$$
\sigma_{p}(P)(\boldsymbol{x}, \boldsymbol{\xi})=\sum_{|\alpha|=m} a_{\alpha}(\boldsymbol{x}) \boldsymbol{\xi}^{\alpha}
$$

For example, for the operator "P(x,D):= $\operatorname{div}\left(A_{2}(\boldsymbol{x}) \nabla \cdot\right)+a_{0}(\boldsymbol{x})$ ", we have $p(\boldsymbol{x}, \boldsymbol{\xi})=-\boldsymbol{\xi}^{T} A_{2}(\boldsymbol{x}) \boldsymbol{\xi}+a_{0}(\boldsymbol{x})$ and $\sigma_{p}(P)(\boldsymbol{x}, \boldsymbol{\xi})=-\boldsymbol{\xi}^{T} A_{2}(\boldsymbol{x}) \boldsymbol{\xi}$. Here, div $\mathbf{v}:=$ 
$\partial_{1} \mathbf{v}_{1}+\ldots+\partial_{d} \mathbf{v}_{d}$ is the usual divergence operator of a vector field $\mathbf{v}$ and $\nabla$ is the gradient operator.

Let $f$ be a function in the Schwartz space $\mathcal{S}$ of $\mathcal{C}^{\infty}$ functions that rapidly decay at infinity. Then, its Fourier transform $\hat{f} \in \mathcal{S}$ is defined by

$$
\hat{f}(\boldsymbol{\xi})=\int_{\mathbb{R}^{d}} e^{-i \boldsymbol{x} \cdot \boldsymbol{\xi}} f(\boldsymbol{x}) d \boldsymbol{x},
$$

and we have

$$
f(\boldsymbol{x})=\frac{1}{(2 \pi)^{d}} \int_{\mathbb{R}^{d}} e^{i \boldsymbol{x} \cdot \boldsymbol{\xi}} \hat{f}(\boldsymbol{\xi}) d \boldsymbol{\xi} .
$$

It is well-known that the Fourier transform is an isomorphism from $\mathcal{S}$. Moreover we have the property $\widehat{D^{\alpha} u}(\boldsymbol{\xi})=\boldsymbol{\xi}^{\alpha} \hat{u}(\boldsymbol{\xi})$. The inverse Fourier transform is used to rewrite the partial differential operator $P: \mathcal{S} \rightarrow \mathcal{S}$ through its symbol $p$ as

$$
P(\boldsymbol{x}, D) u(\boldsymbol{x})=\frac{1}{(2 \pi)^{d}} \int_{\mathbb{R}^{d}} e^{i \boldsymbol{x} \cdot \boldsymbol{\xi}} p(\boldsymbol{x}, \boldsymbol{\xi}) \hat{u}(\boldsymbol{\xi}) d \boldsymbol{\xi},
$$

with $u \in \mathcal{S}$. Pseudodifferential operators are a generalization of differential operators. The motivation is to replace polynomial symbols $p$ in $\boldsymbol{\xi}$ with more general symbols. Working not only locally in space for $\boldsymbol{x}$ but also in the cotangent space with respect to $\boldsymbol{\xi}$ is known as microlocal analysis.

Let us introduce the space of admissible symbols that define a pseudodifferential operator.

Definition 2 Let $m \in \mathbb{R}$. We denote by $\mathcal{S}^{m}(\Omega)$ the space of functions $a \in$ $\mathcal{C}^{\infty}\left(\Omega \times \mathbb{R}^{d}\right)$ such that for every compact subset $K \subseteq \Omega$ and every $\alpha \in \mathbb{N}^{d}$, $\beta \in \mathbb{N}^{d}$, there exists a constant $C=C(K, \alpha, \beta) \in \mathbb{R}$ such that

$$
\left|\partial_{\boldsymbol{x}}^{\beta} \partial_{\boldsymbol{\xi}}^{\alpha} a(\boldsymbol{x}, \boldsymbol{\xi})\right| \leq C(1+|\boldsymbol{\xi}|)^{m-|\alpha|}, \quad \forall(\boldsymbol{x}, \boldsymbol{\xi}) \in K \times \mathbb{R}^{d} .
$$

The notation $|\boldsymbol{\xi}|$ designates the euclidian norm of vector $\boldsymbol{\xi} \in \mathbb{R}^{d}$, i.e. $|\boldsymbol{\xi}|=$ $\sqrt{\boldsymbol{\xi} \cdot \boldsymbol{\xi}}$, and, for a multi-index, we set $|\alpha|=\alpha_{1}+\ldots+\alpha_{d}$. Elements of $\mathcal{S}^{m}(\Omega)$ are called symbols of order $m$ and we write $a \in \mathcal{S}^{m}$.

Let us define now a pseudodifferential operator $A$ of order $m$ through its symbol $a \in \mathcal{S}^{m}$ and the inverse Fourier transform.

Definition 3 A symbol $a \in \mathcal{S}^{m}(\Omega)$ defines a continuous linear operator $A=$ Op $a: \mathcal{C}_{0}^{\infty}(\Omega) \rightarrow \mathcal{C}_{0}^{\infty}(\Omega)$ by

$$
A(\boldsymbol{x}, D) u(\boldsymbol{x})=\frac{1}{(2 \pi)^{d}} \int_{\mathbb{R}^{d}} e^{i \boldsymbol{x} \cdot \boldsymbol{\xi}} a(\boldsymbol{x}, \boldsymbol{\xi}) \hat{u}(\boldsymbol{\xi}) d \boldsymbol{\xi} .
$$

Operators of this type are called pseudodifferential operators of symbol $a$ and of order $m$. We set then $A \in \psi^{m}(\Omega)$. 
Pseudodifferential operators of arbitrarily small order $m$ are called regularizing and we have $\psi^{-\infty}(\Omega)=\cap_{m \in \mathbb{R}} \psi^{m}(\Omega)$. The theory of pseudodifferential operators offers an interesting and useful property through the symbolic calculus. This leads to practical computations on operators working at the algebraic level with their symbols (see section 3.2.

To complete some of the definitions below, we introduce the notion of elliptic pseudodifferential operator.

Definition 4 A symbol $a \in \mathcal{S}^{m}(\Omega)$ is called elliptic (of degree $m$ ) if for every $K \subset \Omega$ compact, there exists a constant $C$ such that

$$
|a(\boldsymbol{x}, \boldsymbol{\xi})| \geq C(1+|\boldsymbol{\xi}|)^{m}, \forall \boldsymbol{x} \in K,|\boldsymbol{\xi}| \geq \frac{1}{C} .
$$

An elliptic pseudodifferential operator $A \in \psi^{m}(\Omega)$ is an operator with an elliptic symbol $a \in \mathcal{S}^{m}(\Omega)$.

Pseudodifferential operators have regularity properties which are related to their order $m$. For example, if we denote by $H^{s}(\Omega)$ the Sobolev space of order $s \in \mathbb{R}$ of distributions $u$ defined on $\Omega[112$, then it can be proved that $A$ is a continuous linear operator acting from $H^{s}(\Omega)$ onto $H^{s-m}(\Omega)$, for any $s \in \mathbb{R}$.

Finally, pseudodifferential operators have the pseudolocal property. We say that an operator $A$ acting on a distribution $u$ is local if $A u$ is smooth in the same set as $u$. Pseudolocal means that the set where $A$ is smooth includes the set where $u$ is smooth. This implies that $A$ could smooth out a nonsmoothness of $u$ (a more rigorous mathematical definition uses the notion of support and singular support of a distribution [112]). Partial differential operators with smooth coefficients are local operators and every local operator is a differential operator. Examples of pseudolocal operators include integral operators $A$ of the form

$$
A u(\boldsymbol{x})=\int_{\Omega} G(\boldsymbol{x}, \boldsymbol{y}) u(\boldsymbol{y}) d \boldsymbol{y}
$$

where $G$ is a smooth kernel.

\subsection{Practical symbolic calculus rules}

The most important property of pseudodifferential operators for practical applications in scientific computing is the fact that all the computations like the composition of two operators or the transposition of an operator can be performed algebraically at the symbol level. We give here the main results useful for the sequel and we most particularly introduce the idea of asymptotic expansion of a symbol.

A symbol $a \in \mathcal{S}^{m}(\Omega)$ is said to be homogeneous of degree $m$ in $\boldsymbol{\xi}$ if the following relation holds

$$
\forall(\boldsymbol{x}, \boldsymbol{\xi}) \in \Omega \times\left(\mathbb{R}^{d} \backslash 0\right), \forall \lambda>0, a(\boldsymbol{x}, \lambda \boldsymbol{\xi})=\lambda^{m} a(\boldsymbol{x}, \boldsymbol{\xi}) .
$$

We can then give the following definition. 
Definition 5 Let $a \in \mathcal{S}^{m}(\Omega)$. Consider a decreasing sequence of real constants $\left(m_{j}\right)_{j=1}^{\infty}$, with $\lim _{j \rightarrow \infty} m_{j}=-\infty$. Let $\left(a_{j}\right)_{j=1}^{\infty}$ be a sequence of homogeneous symbols $a_{j} \in \mathcal{S}^{m_{j}}(\Omega)$ such that

$$
a-\sum_{j=1}^{k} a_{j} \in \mathcal{S}^{m_{k+1}}(\Omega),
$$

for every $k \in \mathbb{N}, k \neq 0$. Then, we say that $\left(a_{j}\right)_{j=1}^{\infty}$ is an asymptotic expansion of $a$. In this case, we write $a \sim \sum_{j} a_{j}$. The first term $a_{1}$ of order $m_{1}$ in the expansion is called the principal symbol.

Not every symbol $a \in \mathcal{S}^{m}(\Omega)$ has an asymptotic expansion. The set of symbols of the form $(11)$ is called classical. It is denoted by $\mathcal{S}_{\mathrm{cl}}^{m}(\Omega)$ and the corresponding operators belong to $\psi_{\mathrm{cl}}^{m}(\Omega)$.

Remark 2 If two symbols $a$ et $b$ have the same asymptotic expansion, then they differ from a smoothing pseudodifferential operator

$$
a-b=\left(a-\sum_{j=1}^{k} a_{j}\right)-\left(b-\sum_{j=1}^{k} a_{j}\right) \in \mathcal{S}^{m_{k+1}}(\Omega),
$$

for all $\mathrm{k}$ and $\lim _{j \rightarrow+\infty} m_{j}=-\infty$, so $(a-b) \in \mathcal{S}^{-\infty}(\Omega)$, with $\mathcal{S}^{-\infty}(\Omega)=$ $\cap_{m \in \mathbb{R}} \mathcal{S}^{m}(\Omega)$.

As said above, one of the crucial points of pseudodifferential operators is that we have algebraic rules for computing some operations on pseudodifferential operators. Two extremely important properties are the following.

Proposition 3 Let $A \in \psi_{c l}^{m_{1}}(\Omega)$ and $B \in \psi_{c l}^{m_{2}}(\Omega)$ with symbols $a \in \mathcal{S}_{c l}^{m_{1}}(\Omega)$ and $b \in \mathcal{S}_{c l}^{m_{2}}(\Omega)$, respectively. Then

1. The transpose $A^{t}$ of $A$ is a pseudodifferential operator. The symbol $a^{t} \in$ $\mathcal{S}_{c l}^{m_{1}}(\Omega)$ of $A^{t} \in \psi_{c l}^{m_{1}}(\Omega)$ is given by the following expansion

$$
a^{t}(\boldsymbol{x}, \boldsymbol{\xi}) \sim \sum_{\alpha \in \mathbb{N}^{d}} \frac{1}{\alpha !} \partial_{\boldsymbol{\xi}}^{\alpha} D_{\boldsymbol{x}}^{\alpha} a(\boldsymbol{x},-\boldsymbol{\xi})
$$

where $\alpha \in \mathbb{N}^{d}$ is a multi-index.

2. The composition of two operators $A$ and $B$, denoted by $A B$, is a pseudodifferential operator. The symbol $a \sharp b \in \mathcal{S}_{c l}^{m_{1}+m_{2}}(\Omega)$ of $A B \in \psi_{c l}^{m_{1}+m_{2}}(\Omega)$ is

$$
a \sharp b(\boldsymbol{x}, \boldsymbol{\xi}) \sim \sum_{\alpha \in \mathbb{N}^{d}} \frac{1}{\alpha !} \partial_{\boldsymbol{\xi}}^{\alpha} a(\boldsymbol{x}, \boldsymbol{\xi}) D_{\boldsymbol{x}}^{\alpha} b(\boldsymbol{x}, \boldsymbol{\xi}) .
$$

In particular, this shows that $a(\boldsymbol{x},-\boldsymbol{\xi})^{t}$ is the principal symbol of $A^{t}$ and $\sigma_{p}(A) \sigma_{p}(B)$ of $A B$.

In addition to Proposition 3 , let us remark that $D_{\boldsymbol{x}}^{\alpha} a \in \mathcal{S}_{\mathrm{cl}}^{m_{1}}$ and $\partial_{\boldsymbol{\xi}}^{\alpha} a \in \mathcal{S}_{\mathrm{cl}}^{m_{1}-|\alpha|}$ if $a \in \mathcal{S}_{\mathrm{cl}}^{m_{1}}$. Furthermore, we have the following Theorem. 
Theorem 3 Let $A$ be an elliptic pseudodifferential operator of order $m$. There exists a pseudodifferential operator $B$ (inverse of $A$ ) with order $-m$ such that $A B-I$ (right inverse of $A$ ) and $B A-I \in \psi^{-\infty}(\Omega)$ (left inverse of $A$ ). The operator $I$ is the identity operator (with symbol 1 ).

We can see that combining formula $(13)$ and Theorem 3 gives a practical way of computing an approximate inverse $B$ of a given pseudodifferential operator $A$ if we know its symbol $a$ or at least the first terms of its asymptotic expansion. In practice, this can often be done. This point of view is at the basis of what we call analytical preconditioners which is an alternative approach to the purely algebraic methods described briefly in section 2.5

3.3 A first and simple application to preconditioning of the Helmholtz equation

An example of application of pseudodifferential operators theory and symbolic calculus rules to preconditioning is the following. Let us consider the Helmholtz operator $L=\Delta+k^{2}$ of symbol $\sigma_{L}(\boldsymbol{x}, \boldsymbol{\xi})=\sigma_{L}(\boldsymbol{\xi})=k^{2}-|\boldsymbol{\xi}|^{2}$ (the dependence in $\boldsymbol{x}$ really occurs for an inhomogeneous media). Then, an exact analytical (left) preconditioner would be an operator $A$ such that $A L=I$. Since $L$ is a pseudodifferential (in fact, partial differential) operator of order $2, A$ is a pseudodifferential operator of order -2 . Formally, $A$ is equal to the nonlocal operator $\left(\Delta+k^{2}\right)^{-1}$ with symbol $\left(k^{2}-|\boldsymbol{\xi}|^{2}\right)^{-1}$. This point of view is purely theoretical since the practical computation of $A$ is exactly what we wish to obtain by solving the initial scattering problem. A first approximation is to consider a static approximation of the symbol of $A$ as: $\sigma_{A} \approx\left(-|\boldsymbol{\xi}|^{2}\right)^{-1}$. This means that the corresponding analytical preconditioner is $A_{0}=O p\left(\left(-|\boldsymbol{\xi}|^{2}\right)^{-1}\right)=\Delta^{-1}$, implying that the associated preconditioned operator to solve is: $A_{0} L=\Delta^{-1}\left(\Delta+k^{2}\right)=I+k^{2} \Delta^{-2}$. Hence $A_{0} L$ is a second-kind integral operator (see Section 4.3.5) with eigenvalue clustering around $(1,0)$ in the complex plane for large frequencies $|\boldsymbol{\xi}|$. This idea was introduced differently by Bayliss, Goldberg and Turkel in 22. It can be shown (see also Figure 8(a) for a one-dimensional example) that this clearly improves the convergence of an iterative solver when considering low-frequencies $k$ (close to the static problem). However, for larger values of $k$ (medium and high frequency regimes), the convergence may fail or strongly depends on $k$. As an example, let us consider $k=60$ and $n_{h}=80$ for the one-dimensional case. We represent on Figure 8(a) the eigenvalue distribution in the complex plane of the corresponding discrete preconditioned matrix $\mathbb{I}-k^{-2} \mathbb{S}_{h}^{-1} \mathbb{M}_{h}$. If we would zoom around the origin, then one would observe a clustering of the eigenvalues around $(1,0)$. However, as we can see, many eigenvalues remain in the left half-plane leading to an indefinite matrix when $k$ is sufficiently large. An improved solution is to consider the smoothing of $A$ by a complex parameter $\alpha \in \mathbb{C}: A_{\alpha}=\left(\Delta+k^{2} \alpha\right)^{-1}$. Adding this parameter leads to the solution of a dissipative Helmholtz equation. This approach is called Laplace 
shifted preconditioning approach [66. In terms of eigenvalues, the symbol of the preconditioned operator $A_{\alpha} L$ is

$$
\sigma_{A_{\alpha} L}(\boldsymbol{x}, z)=\frac{1+z}{\alpha+z}
$$

setting $z=-|\boldsymbol{\xi}|^{2} / k^{2} \in \mathbb{R}^{-}$. The eigenvalues are then distributed along a circular arc as a consequence of the Poincaré map (14). The center of the circle is

$$
\left(\frac{1}{2}, \frac{\left(a^{2}+b^{2}-a\right)}{2 b}\right)
$$

and its radius is

$$
\sqrt{\frac{1}{4}+\frac{\left(a-\left(a^{2}+b^{2}\right)\right)^{2}}{4 b^{2}}}
$$

setting $\alpha=a+i b$. The endpoints are $\alpha^{-1}$ for the low-frequency spatial values $|\boldsymbol{\xi}| \ll k(z \approx 0$ for physical propagative modes) and $(1,0)$ for large frequencies $|\boldsymbol{\xi}| \gg k(z \approx-\infty$ for evanescent waves). For the "grazing" waves related to $|\boldsymbol{\xi}| \approx k(z \approx-1)$, one gets positive eigenvalues close to the origin. These remarks can be observed on Figures 8(b) and 8(c) for respectively $\alpha=1+0.5 i$ and $1+i$ ("analytical arc"). From a practical point of view, $A_{\alpha}$ is computed by a few steps of a multigrid algorithm or by an ILU factorization and not a direct solver (see [66,116]). These preconditioners a priori lead to preconditioned matrices with eigenvalues having positive real parts (and so positive-definite matrices correspond). However, for a scattering problem, a boundary contribution related to the ABC must be considered in the global system matrix and must be included into the preconditioner. On figures 8(b) and 8(c), we draw the numerical eigenvalues of the preconditioned matrices $\mathbb{A}_{\alpha, h}^{i k} \mathbb{L}_{h}$ with ABCs, setting $\mathbb{L}_{h}=\mathbb{S}_{h}-k^{2} \mathbb{M}_{h}-i k \mathbb{B}_{h}$ and $\mathbb{A}_{\alpha, h}^{i k}=\left(\mathbb{S}_{h}-k^{2} \alpha \mathbb{M}_{h}-i k \mathbb{B}_{h}\right)^{-1}$. We observe that the introduction of the boundary term modifies the circular arc. Most particularly, the associated spectrum has eigenvalues with negative real parts, meaning that $\mathbb{A}_{\alpha, h}^{i k} \mathbb{L}_{h}$ is an indefinite matrix now. The residual history of the GMRES(50) for computing the solution to the scattering problem with different preconditioners are reported on Figure 8(d). This shows the divergence of the restarted GMRES without preconditioner and its convergence for $\alpha=0, \alpha=1+i$ and $\alpha=1+0.5 i$, which is compatible with the literature on the topic 66, 116. The fastest convergence is obtained with $\alpha=1+0.5 i$, which can be expected from Figure $8(\mathrm{c})$ since most eigenvalues have significative positive real parts.

This first simple application shows that the pseudodifferential operator theory and symbolic calculus lead to some possible constructions of robust and efficient preconditioners for PDEs. The aim of the next section is to provide other applications for preconditioning well-known integral equation formulations used in acoustic scattering problems. 


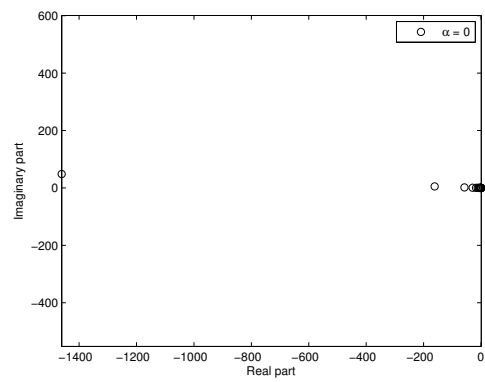

(a) $\alpha=0$

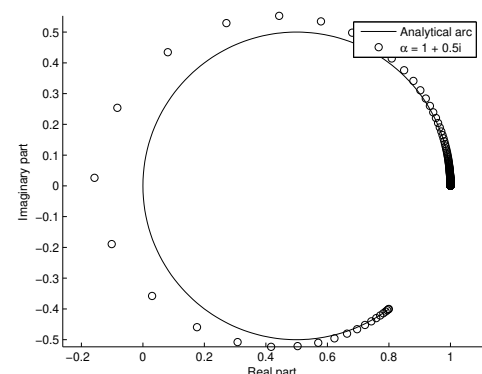

(c) $\alpha=1+0.5 i$

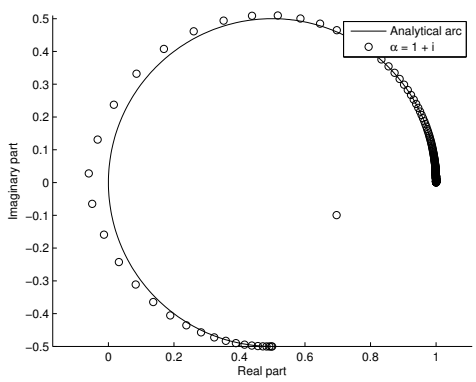

(b) $\alpha=1+i$

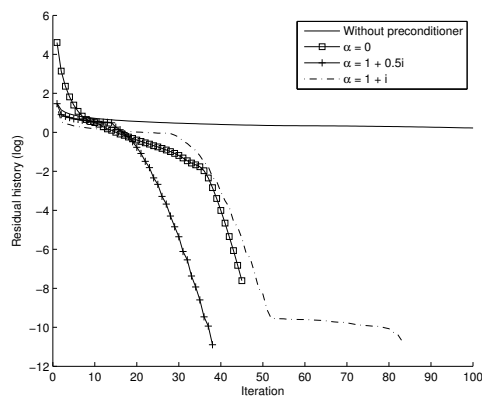

(d) Convergence history

Fig. 8 Laplace shifted preconditioning.

\section{Potential theory - integral equations}

In this section, we present how to solve an exterior boundary value problem set in an unbounded domain via the integral equation method. First, we recall the basic acoustic scattering problem and some notations. Next, we give elements of potential theory that are crucial for the integral equation method. Finally, we discuss the derivation of the classical direct and indirect integral equations for both Dirichlet and Neumann boundary conditions. We also describe their properties for a numerical solution by a Krylov iterative solver (like the GMRES).

\subsection{Acoustic scattering problems}

Let us define a $d$-dimensional bounded domain $\Omega^{-} \subset \mathbb{R}^{d}$ representing a non penetrable body with boundary $\Gamma:=\partial \Omega^{-}$. We denote by $\Omega^{+}:=\mathbb{R}^{d} \backslash \overline{\Omega^{-}}$the associated homogeneous exterior domain of propagation. Consider the scattering of an incident time-harmonic acoustic wave $u^{\text {inc }}$ by the obstacle $\Omega^{-}$. The 
scattered field $u^{+}$satisfies the following exterior boundary-value problem

$$
\left\{\begin{array}{l}
\Delta u^{+}+k^{2} u^{+}=0, \text { in } \Omega^{+}, \\
\left.u^{+}\right|_{\Gamma}=-\left.u^{\mathrm{inc}}\right|_{\Gamma} \text { or }\left.\partial_{\boldsymbol{n}} u^{+}\right|_{\Gamma}=-\left.\partial_{\boldsymbol{n}} u^{\mathrm{inc}}\right|_{\Gamma}, \quad \text { on } \Gamma, \\
\lim _{\|\boldsymbol{x}\| \rightarrow+\infty}\|\boldsymbol{x}\|^{(d-1) / 2}\left(\nabla u^{+} \cdot \frac{\boldsymbol{x} \boldsymbol{x} \|}{\left.\| k u^{+}\right)=0,}\right.
\end{array}\right.
$$

setting $\Delta:=\sum_{i=1}^{d} \partial_{x_{j}}^{2},\|\boldsymbol{x}\|=\sqrt{\boldsymbol{x} \cdot \boldsymbol{x}}$ is the 2-norm of $\boldsymbol{x} \in \mathbb{C}^{d}$ and $\boldsymbol{x} \cdot \boldsymbol{y}$ the inner product of two-complex vectors $\boldsymbol{x}$ and $\boldsymbol{y}$ in $\mathbb{C}^{d}$. We consider an incident time-harmonic plane wave of the form

$$
u^{\text {inc }}(\boldsymbol{x})=e^{-i k \boldsymbol{\theta}^{\mathrm{inc}} \cdot \boldsymbol{x}} .
$$

This wavefield is characterized by the wavenumber $k:=2 \pi / \lambda$, setting $\lambda$ as the wavelength of the signal. In the two-dimensional case $(d=2)$, the direction of incidence $\boldsymbol{\theta}^{\text {inc }}$ is given by the relation $\boldsymbol{\theta}^{\text {inc }}=\left(\cos \left(\theta^{\text {inc }}\right), \sin \left(\theta^{\text {inc }}\right)\right)^{T}$, where $\theta^{\text {inc }}$ is the scattering angle in the polar coordinates system. In the threedimensional case $(d=3)$, we have

$$
\boldsymbol{\theta}^{\mathrm{inc}}=\left(\cos \left(\theta^{\mathrm{inc}}\right) \sin \left(\phi^{\mathrm{inc}}\right), \sin \left(\theta^{\mathrm{inc}}\right) \sin \left(\phi^{\mathrm{inc}}\right), \cos \left(\phi^{\mathrm{inc}}\right)\right)^{T} .
$$

The scattering angles $\left(\theta^{\text {inc }}, \phi^{\text {inc }}\right)$ are expressed in the spherical coordinates system. We define by $\boldsymbol{n}$ the outwardly directed unit normal to $\Omega^{-}$at the boundary $\Gamma$. The boundary condition on $\Gamma$ (second equation of (15p) depends on the physical problem under study. The sound-soft or Dirichlet (respectively sound-hard or Neumann) boundary condition on $\Gamma$ corresponds to the first (respectively second) boundary condition in (15). Finally, the last equation is the well-known Sommerfeld's radiation condition or the outgoing wave condition. This condition models the behaviour of the solution to the Helmholtz equation at infinity and guarantees the uniqueness of the solution to the exterior problem 15.

Let us introduce the functional spaces [100]

$$
\begin{aligned}
& H_{\mathrm{loc}}^{s}\left(\overline{\Omega^{+}}\right):=\left\{v \in \mathcal{D}^{\prime}\left(\Omega^{+}\right) / \psi v \in H^{s}\left(\Omega^{+}\right), \forall \psi \in \mathcal{D}\left(\mathbb{R}^{d}\right)\right\}, \quad s \geq 1, \\
& H_{-}^{1}(\Delta):=H^{1}\left(\Delta, \overline{\Omega^{-}}\right):=\left\{u \in H^{1}\left(\overline{\Omega^{-}}\right) ; \Delta u \in L^{2}\left(\overline{\Omega^{-}}\right)\right\}, \\
& H_{+}^{1}(\Delta):=H_{\mathrm{loc}}^{1}\left(\Delta, \overline{\Omega^{+}}\right):=\left\{u \in H_{\mathrm{loc}}^{1}\left(\overline{\Omega^{+}}\right) ; \Delta u \in L_{\mathrm{loc}}^{2}\left(\overline{\Omega^{+}}\right)\right\} .
\end{aligned}
$$

For $u \in H_{ \pm}^{1}(\Delta)$, the exterior $(+)$ and interior $(-)$ trace operators of order $j$ $(j=0$ or 1$)$ can be defined by

$$
\begin{aligned}
\gamma_{j}^{ \pm}: H_{ \pm}^{1}(\Delta) & \rightarrow H^{1 / 2-j}(\Gamma) \\
u & \mapsto \gamma_{j}^{ \pm} u^{ \pm}=\left.\partial_{\boldsymbol{n}}^{j} u^{ \pm}\right|_{\Gamma} .
\end{aligned}
$$

In this functional setting, the existence and uniqueness of the solution to the scattering problem

$$
\left\{\begin{array}{l}
\text { Find } u^{+} \in H_{\text {loc }}^{1}\left(\overline{\Omega^{+}}\right) \text {such that } \\
\Delta u^{+}+k^{2} u^{+}=0, \text { in } \mathcal{D}^{\prime}\left(\Omega^{+}\right), \\
\gamma_{j}^{+} u^{+}=g:=-\gamma_{j}^{+} u^{\text {inc }}, \quad \text { in } H^{1 / 2-j}(\Gamma), \quad j=0 \text { or } 1, \\
\lim _{\|\boldsymbol{x}\| \rightarrow+\infty}\|\boldsymbol{x}\|^{(d-1) / 2}\left(\nabla u^{+} \cdot \frac{\boldsymbol{x}}{\|\boldsymbol{x}\|}-i k u^{+}\right)=0,
\end{array}\right.
$$


can be proved [56].

The first main difficulty arising in the numerical solution of the exterior boundary-value problem $(17)$ is related to the unboundedness of the propagation domain $\Omega^{+}$. A solution is to apply the integral equation method [51, 56. This approach allows to equivalently formulate the initial boundary-value problem as an integral equation defined on the boundary $\Gamma$ of the obstacle $\Omega^{-}$. Thus, this method reduces the dimension of the problem to $d-1$. Boundary integral equations are derived from the potential theory. Let us give in the following Section some elements of this theory.

4.2 Potential theory: basic relations - properties

The essential property is that any solution to the Helmholtz equation can be represented as the linear combination of a single- and a double-layer potentials. The following proposition holds.

Proposition 4 Let us define the outgoing Green's function $G$ associated with the Helmholtz operator in $\mathbb{R}^{d}$ by

$$
G(\boldsymbol{x}, \boldsymbol{y})=\left\{\begin{array}{l}
\frac{i}{4} H_{0}^{(1)}(k\|\boldsymbol{x}-\boldsymbol{y}\|), \text { for } d=2, \\
\frac{1}{4 \pi} \frac{e^{i k\|\boldsymbol{x}-\boldsymbol{y}\|}}{\|\boldsymbol{x}-\boldsymbol{y}\|}, \text { for } d=3,
\end{array}\right.
$$

where $H_{0}^{(1)}$ designates the first-kind Hankel function of order zero [1]. Let $\left(v^{-}, v^{+}\right) \in H^{1}\left(\Omega^{-}\right) \times H_{\mathrm{loc}}^{1}\left(\overline{\Omega^{+}}\right)$satisfying

$$
\Delta v^{-}+k^{2} v^{-}=0 \text { in } \Omega^{-},
$$

and

$$
\left\{\begin{array}{l}
\Delta v^{+}+k^{2} v^{+}=0 \text { in } \Omega^{+} \\
v^{+} \text {outgoing wave. }
\end{array}\right.
$$

Then, we have

$$
L\left(\left[\partial_{\boldsymbol{n}} v(\boldsymbol{y})\right]_{\Gamma}\right)(\boldsymbol{x})-D\left([v(\boldsymbol{y})]_{\Gamma}\right)(\boldsymbol{x})= \begin{cases}v^{-}(\boldsymbol{x}), & \boldsymbol{x} \in \Omega^{-}, \\ v^{+}(\boldsymbol{x}), & \boldsymbol{x} \in \Omega^{+},\end{cases}
$$

where

$$
[v]_{\Gamma}:=\gamma_{0}^{-} v^{-}-\gamma_{0}^{+} v^{+},\left[\partial_{\boldsymbol{n}} v\right]_{\Gamma}:=\gamma_{1}^{-} v^{-}-\gamma_{1}^{+} v^{+},
$$

and

$$
\begin{gathered}
L p(\boldsymbol{x}):=\int_{\Gamma} G(\boldsymbol{x}, \boldsymbol{y}) p(\boldsymbol{y}) d \Gamma(\boldsymbol{y}), \quad \boldsymbol{x} \notin \Gamma, \\
D \phi(\boldsymbol{x}):=\int_{\Gamma} \partial_{\boldsymbol{n}(\boldsymbol{y})} G(\boldsymbol{x}, \boldsymbol{y}) \phi(\boldsymbol{y}) d \Gamma(\boldsymbol{y}), \quad \boldsymbol{x} \notin \Gamma,
\end{gathered}
$$

for $(p, \phi) \in H^{1 / 2}(\Gamma) \times H^{-1 / 2}(\Gamma)$. 
The operators $L$ and $D$ defined above are called the single- and the doublelayer potentials respectively. To obtain integral equations set on the boundary $\Gamma$, we need the trace formulae for these two potentials (see for instance [100]).

Proposition 5 The first and second traces on $\Gamma$ of the single- and doublelayer potentials are given by composition (०) as

$$
\left\{\begin{array}{l}
\gamma_{0}^{-} \circ L=\gamma_{0}^{+} \circ L=\mathcal{L}, \\
\gamma_{1}^{\mp} \circ L= \pm \frac{\mathcal{I}}{2}+\mathcal{N},
\end{array}\right.
$$

and

$$
\left\{\begin{array}{l}
\gamma_{0}^{\mp} \circ D=\mp \frac{\mathcal{I}}{2}+\mathcal{D}, \\
\gamma_{1}^{-} \circ D=\gamma_{1}^{+} \circ D=\mathcal{S},
\end{array}\right.
$$

where $\mathcal{I}$ is the identity operator and $\mathcal{L}, \mathcal{N}, \mathcal{D}$ and $\mathcal{S}$ are the four elementary boundary integral operators expressed, for all $\boldsymbol{x} \in \Gamma$, by

$$
\begin{aligned}
\mathcal{L} p(\boldsymbol{x}) & :=\int_{\Gamma} G(\boldsymbol{x}, \boldsymbol{y}) p(\boldsymbol{y}) d \Gamma(\boldsymbol{y}), \\
\mathcal{N} p(\boldsymbol{x}) & :=\int_{\Gamma} \partial_{\boldsymbol{n}(\boldsymbol{x})} G(\boldsymbol{x}, \boldsymbol{y}) p(\boldsymbol{y}) d \Gamma(\boldsymbol{y}), \\
\mathcal{D} \phi(\boldsymbol{x}) & :=\int_{\Gamma} \partial_{\boldsymbol{n}(\boldsymbol{y})} G(\boldsymbol{x}, \boldsymbol{y}) \phi(\boldsymbol{y}) d \Gamma(\boldsymbol{y}), \\
\mathcal{S} \phi(\boldsymbol{x}) & :=\oint_{\Gamma} \frac{\partial^{2} G}{\partial \boldsymbol{n}(\boldsymbol{x}) \partial \boldsymbol{n}(\boldsymbol{y})}(\boldsymbol{x}, \boldsymbol{y}) \phi(\boldsymbol{y}) d \Gamma(\boldsymbol{y}) .
\end{aligned}
$$

Note that the expression defining $\mathcal{S}$ is not an integral (its singularity is not integrable) but a finite part expression associated with a hypersingular kernel. We preferred to keep formally the integral expression for the sake of clarity.

Let us now summarize the continuity properties of the elementary boundary integral operators (see for instance [100, Theorem 4.4.1] or Theorems 7.1 and 7.2 in 95.).

Proposition 6 For a smooth boundary $\Gamma$, the boundary integral operators given in Proposition 5 define the following continuous mappings

$$
\begin{aligned}
& \mathcal{L}: H^{s}(\Gamma) \longrightarrow H^{s+1}(\Gamma), \\
& \mathcal{N}: H^{s}(\Gamma) \longrightarrow H^{s}(\Gamma), \\
& \mathcal{D}: H^{s}(\Gamma) \longrightarrow H^{s}(\Gamma), \\
& \mathcal{S}: H^{s}(\Gamma) \longrightarrow H^{s-1}(\Gamma),
\end{aligned}
$$

for all $s \in \mathbb{R}$. Moreover, the operators $\mathcal{N}$ and $\mathcal{D}$ are compact from $H^{s}(\Gamma)$ onto itself, for all $s \in \mathbb{R}$.

In the case of a Lipschitz boundary [57, 95, the above continuity properties still hold for $-1 \leq s \leq 0$ (respectively for $0 \leq s \leq 1$ ) for the operators $\mathcal{L}$ and $\mathcal{N}$ (respectively $\mathcal{D}$ and $\mathcal{S}$ ), while the compactness properties of $\mathcal{N}$ and $\mathcal{D}$ fails. A possible approach to rigorously extend the following developments is to use some regularizing techniques (e.g. [38, 39,65]). 
The integral representation 190 allows to determine the near-field around the scatterer. Another physical quantity of interest is the scattering amplitude (called also the far-field pattern). For instance, the expression of the scattering amplitude in the direction $\boldsymbol{\theta}$ for the two-dimensional case is given by

$$
a_{0}(\boldsymbol{\theta})=\frac{i}{4} \sqrt{\frac{2}{i \pi k}} \int_{\Gamma} e^{-i k \boldsymbol{y} \cdot \boldsymbol{\theta}}\left(\gamma_{1}^{+} u^{+}(\boldsymbol{y})+i k \boldsymbol{\theta} \cdot \boldsymbol{n}(\boldsymbol{y}) \gamma_{0}^{+} u^{+}(\boldsymbol{y})\right) d \Gamma(\boldsymbol{y}) .
$$

\subsection{Standard integral equations formulations}

The Helmholtz representation formula (19) leads to the construction of many integral equations (equivalent if invertible) in the case of a closed surface. In the case of an open surface, only one integral equation can be written. The aim of this part is to introduce the most standard integral equations for both Dirichlet and Neumann boundary conditions. We usually distinguish between direct and indirect integral equations with their own mathematical properties.

Let us introduce the following notations

- $K_{D}\left(\Omega^{-}\right)=\left\{k_{m}^{D}, m \in \mathbb{N}\right\}$ : the set of Dirichlet irregular frequencies (interior Dirichlet eigenvalues) is the set of values of $k$ such that the boundary value problem

$$
\begin{cases}-\Delta v=k^{2} v, & \text { in } \Omega^{-}, \\ \gamma_{0}^{-} v=0, & \text { on } \Gamma,\end{cases}
$$

admits a non vanishing solution.

- $K_{N}\left(\Omega^{-}\right)=\left\{k_{m}^{N}, m \in \mathbb{N}\right\}$ : the set of Neumann irregular frequencies (interior Neumann eigenvalues) is the set of values of $k$ such that the boundary value problem

$$
\begin{cases}-\Delta v=k^{2} v, & \text { in } \Omega^{-}, \\ \gamma_{1}^{-} v=0, & \text { on } \Gamma,\end{cases}
$$

admits a non vanishing solution.

\subsubsection{The Dirichlet problem: direct integral formulations}

The total field $w$ is expressed by $w:=u^{+}+u^{\text {inc }}$. The direct formulations consist in seeking the total field under the form

$$
w(\boldsymbol{x})=L p(\boldsymbol{x})+u^{\mathrm{inc}}(\boldsymbol{x}), \quad \boldsymbol{x} \in \Omega^{+} .
$$

The integral representation $(26)$ ensures that $w$ is solution to the Helmholtz equation in $\Omega^{-} \cup \Omega^{+}$, and satisfies the Sommerfeld radiation condition. Then, we have to determine the unknown $p$ such that $w$ satisfies also the Dirichlet boundary condition $\left(\gamma_{0}^{+} w=0\right)$. More precisely, the representation 26 ( corresponds to the particular choice of solutions $\left(v^{-}, v^{+}\right):=\left(-u^{\text {inc }}, u^{+}\right)$in Proposition 4 , i.e.

$$
[v]_{\Gamma}=0 \quad\left[\partial_{\mathbf{n}} v\right]_{\Gamma}=-\gamma_{1}^{+} w_{\mid \Gamma}:=p
$$


and

$$
L p(\boldsymbol{x})= \begin{cases}-u^{\mathrm{inc}}(\boldsymbol{x}) & \boldsymbol{x} \in \Omega^{-}, \\ u^{+}(\boldsymbol{x}) & \boldsymbol{x} \in \Omega^{+} .\end{cases}
$$

Then, we get the following single-layer potential representation of the total field

$$
L p(\boldsymbol{x})+u^{\mathrm{inc}}(\boldsymbol{x})= \begin{cases}0 & \text { for } \boldsymbol{x} \in \Omega^{-}, \\ w(\boldsymbol{x}) & \text { for } \boldsymbol{x} \in \Omega^{+} .\end{cases}
$$

This formulation is equivalent to extending artificially the total field by zero inside $\Omega^{-}$, which explains that this approach is also referred sometimes to as the null field method.

The next step is to obtain an integral equation for the physical unknown $p=-\gamma_{1}^{+} w_{\mid \Gamma} \in H^{-1 / 2}(\Gamma)$. To achieve this, the idea is to apply a trace operator to the relation

$$
L p(\boldsymbol{x})+u^{\text {inc }}(\boldsymbol{x})=0, \quad \forall \boldsymbol{x} \in \Omega^{-} .
$$

At this point, many choices are available. Let us cite three of them leading to classical integral equations of potential theory.

- EFIE : This equation is obtained by applying the trace operator $\gamma_{0}^{-}$to 27). Thanks to the trace relations of Proposition 5, this leads to the wellknown Electric Field Integral Equation (EFIE)

$$
\mathcal{L} p=-\gamma_{0}^{+} u^{\text {inc }}, \quad \text { on } \Gamma \text {. }
$$

- MFIE : This equation is derived by taking the normal trace operator $\gamma_{1}^{-}$ to (27). The trace relations of Proposition 5 give the so-called Magnetic Field Integral Equation (MFIE)

$$
\left(\frac{\mathcal{I}}{2}+\mathcal{N}\right) p=-\gamma_{1}^{+} u^{\text {inc }}, \quad \text { on } \Gamma
$$

- CFIE : This last equation is based on the application of the Fourier-Robin (impedance) trace operator $\gamma_{1}^{-}+\eta \gamma_{0}^{-}$to 27 , with $\eta \neq 0$. Once again, using the trace relations of Proposition 5. we get the Combined Field Integral Equation (CFIE)

$$
\left(\frac{\mathcal{I}}{2}+\mathcal{N}+\eta \mathcal{L}\right) p=-\left(\gamma_{1}^{+} u^{\text {inc }}+\eta \gamma_{0}^{+} u^{\text {inc }}\right), \quad \text { on } \Gamma .
$$

The existence and uniqueness results for the above direct integral equations $(\sqrt{28}),(29)$ or $(30))$ are given in the following theorem.

Theorem 4 The following properties hold

1. The EFIE operator $\mathcal{L}$ defines an isomorphism from $H^{-1 / 2}(\Gamma)$ onto $H^{1 / 2}(\Gamma)$ if and only if $k \notin K_{D}\left(\Omega^{-}\right)$. Under this condition, the EFIE (28) is uniquely solvable in $H^{-1 / 2}(\Gamma)$. 
2. The MFIE operator

$$
\frac{\mathcal{I}}{2}+\mathcal{N}
$$

is an isomorphism from $H^{-1 / 2}(\Gamma)$ onto $H^{-1 / 2}(\Gamma)$ if and only if we have $k \notin K_{N}\left(\Omega^{-}\right)$. For this condition, the MFIE (29) is uniquely solvable in $H^{-1 / 2}(\Gamma)$.

3. The CFIE operator

$$
\frac{\mathcal{I}}{2}+\mathcal{N}+\eta \mathcal{L}
$$

defines an isomorphism from $H^{-1 / 2}(\Gamma)$ onto $H^{-1 / 2}(\Gamma)$, for all wavenumbers $k>0$, if and only if the imaginary part of $\eta$ is such that $\Im(\eta) \neq 0$. Under this condition, then the CFIE (30) is uniquely solvable in $H^{-1 / 2}(\Gamma)$, for any $k>0$.

In the case where $k$ in an irregular frequency, the integral equations EFIE and MFIE have non zero kernels. Nevertheless, it can be shown that the spurious modes of the EFIE will not radiate in the exterior. Thus, the field is not corrupted outside the object: $\mathcal{L} p=0$ on $\Gamma$ implies that $L p=0$ in $\Omega^{+}$. Therefore, the EFIE provides an accurate computation and often represents a reference solution. Unlike the EFIE, the spurious solutions of the MFIE radiate in the exterior domain, leading hence to a wrong solution. Finally, by its construction itself, the CFIE is free of the internal-resonance problem. We consider in the sequel the coupling parameter $\eta=-i k \beta /(1-\beta), \beta \in] 0,1[$, and the CFIE reads

$$
(1-\beta) \frac{i}{k}\left(\frac{\mathcal{I}}{2}+\mathcal{N}\right)+\beta \mathcal{L}=-\left((1-\beta) \frac{i}{k} \gamma_{1}^{+} u^{\mathrm{inc}}+\beta \gamma_{0}^{+} u^{\mathrm{inc}}\right), \quad \text { on } \Gamma .
$$

A common choice of $\beta$ for engineering computations is $\beta=0.2$ which gives an almost minimal condition number for the CFIE.

\subsubsection{The Dirichlet problem: indirect integral formulations}

The indirect formulations are based on the assumption that the solution can be expressed in terms of a source density function defined on the boundary. The unknowns are then generally non-physical quantities. The physical variables are solved afterwards in terms of these source densities. Here, we focus on the most commonly used indirect integral formulation independently proposed by Burton-Miller [40] and Brakhage-Werner [31]. The idea is to seek the exterior field as a superposition of the single- and double-layer potentials acting on a fictitious surface density $\psi$

$$
u^{+}(\boldsymbol{x})=(D+\eta L) \psi(\boldsymbol{x}), \quad \forall \boldsymbol{x} \in \Omega^{+},
$$

where $\eta$ is a complex-valued coupling parameter to choose. The above expression leads, thanks to the trace relations (5), to the following integral equation

$$
\left(\frac{\mathcal{I}}{2}+\mathcal{D}+\eta \mathcal{L}\right) \psi=-\gamma_{0}^{+} u^{\text {inc }}, \quad \text { on } \Gamma .
$$


Considering the above integral equation in the space $H^{1 / 2}(\Gamma)$, we can prove the following result.

Theorem 5 The operator

$$
\frac{\mathcal{I}}{2}+\mathcal{D}+\eta \mathcal{L}
$$

defines an isomorphism from $H^{1 / 2}(\Gamma)$ onto $H^{1 / 2}(\Gamma)$ for all $k>0$ under the condition $\Im(\eta) \neq 0$. Then, 33 is uniquely solvable in $H^{1 / 2}(\Gamma)$, for any frequency $k>0$.

An almost optimal value of $\eta$ has been obtained in [5, 87, 89] as: $\eta=i k$.

\subsubsection{The Neumann problem: direct integral formulations}

Let us now briefly discuss the derivation of direct integral equations in the case of a Neumann boundary condition. The total field $w:=u^{+}+u^{\text {inc }}$ is searched under the form

$$
w(\boldsymbol{x})=D \phi(\boldsymbol{x})+u^{\mathrm{inc}}(\boldsymbol{x}), \quad \boldsymbol{x} \in \Omega^{+} .
$$

Proposition 4 for $\left(v^{-}, v^{+}\right):=\left(-u^{\text {inc }}, u^{+}\right)$leads to

$$
[v]_{\Gamma}=-\gamma_{0}^{+} w:=\phi,\left[\partial_{\mathbf{n}} v\right]_{\Gamma}=0,
$$

and

$$
D \phi(\boldsymbol{x})= \begin{cases}-u^{\mathrm{inc}}(\boldsymbol{x}) & \boldsymbol{x} \in \Omega^{-}, \\ u^{+}(\boldsymbol{x}) & \boldsymbol{x} \in \Omega^{+} .\end{cases}
$$

Then, we get

$$
D \phi(\boldsymbol{x})+u^{\text {inc }}(\boldsymbol{x})= \begin{cases}0 & \text { for } \boldsymbol{x} \in \Omega^{-}, \\ w(\boldsymbol{x}) & \text { for } \boldsymbol{x} \in \Omega^{+} .\end{cases}
$$

Applying a trace operator to the relation

$$
D \phi(\boldsymbol{x})+u^{\mathrm{inc}}(\boldsymbol{x})=0, \quad \forall \boldsymbol{x} \in \Omega^{-},
$$

the physical unknown $\phi=-\gamma_{0}^{+} w \in H^{1 / 2}(\Gamma)$ is solution to the following direct integral equations:

- EFIE :

$$
\mathcal{S} \phi=-\gamma_{1}^{+} u^{\text {inc }}, \quad \text { on } \Gamma \text {. }
$$

- MFIE :

$$
\left(-\frac{\mathcal{I}}{2}+\mathcal{D}\right) \phi=-\gamma_{0}^{+} u^{\text {inc }}, \quad \text { on } \Gamma
$$

- CFIE :

$$
\left(-\frac{\mathcal{I}}{2}+\mathcal{D}+\eta \mathcal{S}\right) \phi=-\left(\eta \gamma_{1}^{+} u_{\mid \Gamma}^{\mathrm{inc}}+\gamma_{0}^{+} u^{\mathrm{inc}}\right), \quad \text { on } \Gamma .
$$

The existence and uniqueness results for the above integral equations are summarized in the next result. 
Theorem 6 The following properties hold

1. The EFIE operator $\mathcal{S}$ defines an isomorphism from $H^{1 / 2}(\Gamma)$ onto $H^{-1 / 2}(\Gamma)$ if and only if $k \notin K_{N}\left(\Omega^{-}\right)$. Under this condition, the EFIE (36) is uniquely solvable in $H^{1 / 2}(\Gamma)$.

2. The MFIE operator

$$
-\frac{\mathcal{I}}{2}+\mathcal{D}
$$

is an isomorphism from $H^{1 / 2}(\Gamma)$ onto $H^{1 / 2}(\Gamma)$ if and only if $k \notin K_{D}\left(\Omega^{-}\right)$. Then, the MFIE (37) is uniquely solvable in $H^{1 / 2}(\Gamma)$.

3. The CFIE operator

$$
-\frac{\mathcal{I}}{2}+\mathcal{D}+\eta \mathcal{S}
$$

is an isomorphism from $H^{1 / 2}(\Gamma)$ onto $H^{-1 / 2}(\Gamma)$, for all wavenumbers $k>$ 0 such that $\Im(\eta) \neq 0$. Under this condition, the CFIE (38) is uniquely solvable in $H^{1 / 2}(\Gamma)$ for all frequencies $k>0$.

The reference CFIE that we consider in the present paper reads

$$
\left((1-\beta) \frac{i}{k}\left(-\frac{\mathcal{I}}{2}+\mathcal{D}\right)-\frac{\beta}{k^{2}} \mathcal{S}\right) \phi=-\left((1-\beta) \frac{i}{k} \gamma_{0}^{+} u^{\mathrm{inc}}-\frac{\beta}{k^{2}} \gamma_{1}^{+} u^{\mathrm{inc}}\right), \quad \text { on } \Gamma \text {. }
$$

\subsubsection{The Neumann problem: indirect integral formulations}

The Brakhage-Werner (or Burton-Miller) integral representation of the exterior field is expressed by

$$
u^{+}(\boldsymbol{x})=(L+\eta D) \varphi(\boldsymbol{x}), \quad \forall \boldsymbol{x} \in \Omega^{+},
$$

where $\eta$ is a complex-valued coupling parameter to determine. Then, the field (40) solves the exterior boundary-value problem (17) if the surface density $\varphi$ is solution to the following integral equation

$$
\left(-\frac{\mathcal{I}}{2}+\mathcal{N}+\eta \mathcal{S}\right) \varphi=-\gamma_{1}^{+} u^{\text {inc }}
$$

called the Burton-Miller or Brakhage-Werner (BW) integral equation. We have the following existence and uniqueness result.

Theorem 7 The operator

$$
-\frac{\mathcal{I}}{2}+\mathcal{N}+\eta \mathcal{S}
$$

defines an isomorphism from $H^{1 / 2}(\Gamma)$ onto $H^{-1 / 2}(\Gamma)$ for all $k>0$, assuming that $\Im(\eta) \neq 0$. Then, the equation (41) is uniquely solvable in $H^{1 / 2}(\Gamma)$ for all strictly positive frequencies $k>0$.

An almost optimal value of $\eta$ has been numerically discussed in [5, 87, 89, as: $\eta=1 / i k$. We will see in Section 8 that better choices can be proposed. 


\subsubsection{First- versus second-kind Fredholm integral equations}

For surface formulations of acoustic scattering problems, we have seen in the previous sections that various integral equations, direct or indirect, combined or not, can be employed. Let us underline that all these integral equations are applicable to closed geometries, and that the EFIE (28) and (36) in the Dirichlet and Neumann cases, respectively) is the only choice for the solutions of scattering problems by open surfaces.

In view of the iterative solution (by the GMRES for instance) of the corresponding linear systems after discretization, two properties are essential to achieve a fast convergence rate: on the one hand the existence and uniqueness of the solution, on the other hand the good clustering of the eigenvalues around an accumulation point far from the origin (and hence a well-conditioning). The first property, as we have seen, is not ensured for each integral equation. Only combined field integral equations (CFIE, BW) provide a well-possedness for any frequency. To observe the second property, let us recall some definitions. Given an integral operator $\mathcal{A} \in \mathcal{L}(X)$ on a Hilbert space $X$, an integral equation is called of first-kind if it is of the form

$$
\mathcal{A} \varrho=f
$$

or second-kind if it is written as

$$
(\mathcal{I}+\mathcal{A}) \varrho=f .
$$

Moreover, if $\mathcal{A}: X \rightarrow X$ is compact, the above equations are respectively called Fredholm integral equations of the first-kind and second-kind. As classically known 88, the spectrum of compact operators is composed in the infinite dimensional case of 0 and a sequence of discrete eigenvalues possibly accumulating at the origin. Therefore, second-kind Fredholm integral equations have large clusters of eigenvalues accumulating at the real value point 1 (cf. Section 2.3). This is a very interesting spectral property which guarantees fast convergence of the Krylov iterative solvers.

The integral operator $\mathcal{L}$ is bounded from $H^{s}(\Gamma)$ onto $H^{s+1}(\Gamma)$, and it is compact from $L^{2}(\Gamma)$ onto itself. Therefore, the EFIE $\sqrt{28}$ is a first-kind Fredholm integral equation on $L^{2}(\Gamma)$ in the case of a Dirichlet condition. On the contrary, the MFIE (29), the CFIE (30) and the BW integral equation (33) are all second-kind integral equations. Then, the combined field integral equations have all the properties required for an efficient iterative solving.

In the case of a Neumann boundary condition, the situation is more complex. In fact, only the MFIE is a second-kind Fredholm integral equation but it is unfortunately ill-posed. The EFIE, CFIE and BW integral equations involve the operator $\mathcal{S}$ which is a first-order, strongly singular and non-compact operator. Therefore, these equations are of the first-kind. There are two alternatives to expect an eigenvalue clustering. The first one is to precondition the integral operator (see Section 7). The second possibility is to incorporate a suitable operator of order -1 which has a regularizing effect on $\mathcal{S}$ and leads 
to a second-kind integral equation (see Section 8). Let us note that in the case of the numerical solution of scattering problems by an open surface, the preconditioning represents the only way to improve convergence. We will come back to this point in Section 7 .

\section{Developing fast converging solutions: strategy and problematics}

Let us consider now an integral equation representation

$$
A \varrho=f
$$

that we want to numerically solve. Here, $A$ is one of the previous first- or second-kind integral operators, $f$ is a right-hand side given by the incident field and $\varrho$ is the density that we would like to compute. For a numerical calculation, the surface of the scatterer needs to be discretized by using for example $N_{K}$ triangles $K$ in three-dimensions, resulting in a discrete surface $\Gamma_{h}=\cup_{j=1}^{N_{K}} K$, where $h$ is the meshsize (see Section 8.3 for more details). Let us consider a regular triangulation $\mathcal{T}_{h}$ based on triangles. The linear Galerkin BEM uses the approximation space

$$
V_{h}=\left\{v_{h} \in C^{0}\left(\Gamma_{h}\right) ; v_{h}=\left.v_{h}\right|_{\Gamma} \in \mathbb{P}_{1}, \forall T \in \mathcal{T}_{h}\right\},
$$

of dimension $N_{V}$ (equal to the number of degrees of freedom). The density of discretization points per wavelengh $n_{\lambda}$ is given by $n_{\lambda}=\lambda / h_{\max }$, where $h_{\max }$ is the maximal length of the edges of the triangles. Then, the operator $A$ is also approximated by a matrix $[A]$ and the right hand-side $f$ by a complexvalued vector $\mathbf{f}_{h}$. The sizes of the vector and the matrix $[A]$ are equal to the number of degrees of freedom of the BEM used to approximate the density $\varrho$ by a vector $\varrho_{h}$. For example, using linear boundary elements leads to $N_{V}$ degrees of freedom, where $N_{V}$ is the number of vertices of the triangular mesh. One problem that we do not address here but which is a hard task in integral equations consists in integrating the kernel singularities. However, since it is out of our goal, we do not develop this point here.

Consider now that we want to solve the linear system

$$
[A] \varrho_{h}=\mathbf{f}_{h} .
$$

The matrix $[A]$ is complex-valued, dense and highly non-definite positive. It requires a storage of the order of $\mathcal{O}\left(N_{V}^{2}\right)$ (if a linear BEM is used) and its solution by a direct gaussian solver requires $\mathcal{O}\left(N_{V}^{3}\right)$ operations. For high frequency problems, then $N_{V}$ becomes extremely large and makes the direct approach not applicable. Therefore, since the introduction of the FMM [51,55, 62, 63, 70, 82, 105, 111, the strategy for solving an integral equation has fundamentally changed. The FMM (as well as other similar techniques, see [34, 35, 36]) allows to compute with a low-storage $\mathcal{O}\left(N_{V}\right)$ and in a fast way $\mathcal{O}\left(N_{V} \log N_{V}\right)$ the MVP: $\mathbf{x}_{h} \rightarrow[A] \mathbf{x}_{h}$ ("black box"). This matrix-free approach permits to solve higher frequency problems if it is coupled with an iterative Krylov solver like 
the GMRES. As previously noticed, the global algorithm is efficient and robust if the GMRES converges with a few iterations. This is closely related to the spectral properties of $A$ as well as the way a preconditioner is built. Since we have a non positive-definite matrix, the iterative algorithm may diverge. If not, its convergence can be extremely slow. Furthermore, since we do not have the matrix at hand (because of the matrix-free FMM approach), building the preconditioner must be done without having access to the whole matrix.

The aim of the next sections are 1) to propose a spectral analysis of integral operators in some simplified situations to understand their properties (Section 6) and 2) to explain two recent matrix-free analytical preconditioning techniques (Sections 7 and 8) for solving integral equation formulations in acoustic.

\section{Spectral analysis of integral operators for the sphere}

Let $\Gamma=S_{1}$ be the unit sphere centered at the origin. Let us introduce the spherical harmonics $Y_{m}^{n}$ as the functions of order $m$ for $n=-m \cdots m$, with $m \in \mathbb{N}$ [56], given by

$$
Y_{m}^{n}\left(\theta_{1}, \theta_{2}\right)=\sqrt{\frac{2 m+1}{4 \pi} \frac{(m-|n|) !}{(m+|n|) !}} P_{m}^{n}\left(\cos \theta_{1}\right) e^{i n \theta_{2}}, \quad\left(\theta_{1}, \theta_{2}\right) \in S_{1},
$$

where $P_{m}^{n}$ are the Legendre polynomials. The functions $Y_{m}^{n}$ form a complete orthonormal system of $L^{2}\left(S_{1}\right)$. Furthermore, they also constitute a basis of eigenvectors for the four elementary integral operators $\mathcal{L}, \mathcal{N}, \mathcal{D}$ and $\mathcal{S}$ (cf. proposition 5). More precisely, we have the following Proposition [6, 87].

Proposition 7 The eigenvalues $\mathcal{L}_{m}, \mathcal{N}_{m}, \mathcal{D}_{m}$ and $\mathcal{S}_{m}$ of multiplicity $(2 m+1)$ respectively associated with the elementary integral operators $\mathcal{L}, \mathcal{N}, \mathcal{D}$ and $\mathcal{S}$ are given by

$$
\begin{aligned}
\mathcal{L} Y_{m}^{n} & =\left\{i k j_{m}(k) h_{m}^{(1)}(k)\right\} Y_{m}^{n}=\mathcal{L}_{m} Y_{m}^{n}, \\
\mathcal{N} Y_{m}^{n} & =\left\{-1 / 2+i k^{2} j_{m}^{\prime}(k) h_{m}^{(1)}(k)\right\} Y_{m}^{n}=\mathcal{N}_{m} Y_{m}^{n}, \\
\mathcal{D} Y_{m}^{n} & =\mathcal{N}^{t} Y_{m}^{n}=\mathcal{N}_{m} Y_{m}^{n}=\mathcal{D}_{m} Y_{m}^{n}, \\
\mathcal{S} Y_{m}^{n} & =\left\{i k^{3} j_{m}^{\prime}(k)\left(h_{m}^{(1)}\right)^{\prime}(k)\right\} Y_{m}^{n}=\mathcal{S}_{m} Y_{m}^{n},
\end{aligned}
$$

where $j_{m}$ denotes the spherical Bessel function and $h_{m}^{(1)}$ the spherical Hankel function of the first-kind [1]. Their derivatives are specified with a prime'.

Let us begin with the case of a sound-soft sphere. We consider the EFIE operator $\mathcal{L}(28)$, the CFIE operator (31) and the usual BW operator (33) with $\eta=i k$. A direct computation gives the eigenvalues $\mathcal{C}_{m}^{D}$ and $\mathcal{B}_{m}^{D}$ of the combined operators (31) and (33) respectively

$$
\begin{gathered}
\mathcal{C}_{m}^{D}=i k^{2} h_{m}^{(1)}(k)\left(\beta j_{m}^{\prime}(k)+i(1-\beta) j_{m}(k)\right), \\
\mathcal{B}_{m}^{D}=i k^{2} h_{m}^{(1)}(k)\left(j_{m}^{\prime}(k)-i j_{m}(k)\right) .
\end{gathered}
$$


We report on Figure 9 the spectrum of the usual integral operators taking $k=30$ and a maximal number of modes $m_{\max }=12 k$. To understand the spectral distribution, we need to introduce three zones: the elliptic zone of evanescent modes $(m \gg k)$, the hyperbolic region of propagative modes ( $m \ll k$ ) and the transition (hyperbolic-elliptic) zone of physical surface modes satisfying $m \approx k$. To make the connection with pseudodifferential operators, think for easiness that $|\xi|=m$. For the EFIE (Figure $9(\mathrm{a})$ ), we can see that some eigenvalues spread out in the complex plane and are related to the finite number of propagative modes. Next, a loop begins to appear for modes close to the transition zone. Finally, we observe an accumulation point at $(0,0)$ for large modes $m$. Since $\mathcal{L}$ is a first-kind operator of order -1 , its eigenvalues asymptotically behave like $m^{-1}$ for large values of $m$ (elliptic zone). This implies that the smallest eigenvalue is related to the largest mode $m_{\max }$ and makes appear a dependence of the condition number according to the density of discretization points per wavelength $n_{\lambda}$ for integral equations [51. The largest eigenvalue is related to a propagative mode and leads to a dependence of the condition number according to $k$. Therefore, the EFIE has a spectrum which is not really appropriate to an iterative solver and preconditioning will have to be considered, most particularly for large wavenumbers $k$ and large densities of discretization points. Two large clusters of eigenvalues at points $(1 / 2,0)$ and $(1,0)$ can be observed for the BW operator (Figure $9(\mathrm{~b})$ ). The first appears in the elliptic zone and the second one in the hyperbolic zone. The few eigenvalues that form the loop between low-order and high-order modes $m$ correspond to surface modes. The CFIE operator also offers an interesting and quite similar distribution of eigenvalues (Figure $9(\mathrm{c})$ ). These good spectral properties are linked to the Fredholm second-kind character of these operators. As a consequence, the standard CFIE and BW formulations are well-adapted for the iterative solution of the acoustic sound-soft problem.

Let us now consider the hard sphere. The eigenvalues $\mathcal{C}_{m}^{N}$ and $\mathcal{B}_{m}^{N}$ of the CFIE $(39)$ and the BW $(\sqrt{33}), \eta=i / k)$ operators are expressed respectively by

$$
\begin{gathered}
\mathcal{C}_{m}^{N}=k\left(h_{m}^{(1)}\right)^{\prime}(k)\left((1-\beta) j_{m}(k)+i \beta j_{m}^{\prime}(k)\right), \\
\mathcal{B}_{m}^{N}=-i k^{2}\left(h_{m}^{(1)}\right)^{\prime}(k)\left(j_{m}(k)+i j_{m}^{\prime}(k)\right) .
\end{gathered}
$$

We draw on Fig. 10 the distribution of the eigenvalues for the EFIE, BW and CFIE operators. We fix $k=30$ and $m_{\max }=12 k$ modes. The EFIE operator is of order 1 and its eigenvalues associated with high order modes become large since they behave like $m$ (Figure 10(a)). The smallest eigenvalue is associated with the propagative modes. For the usual BW integral operator (Figure 10(b)], we observe a cluster of eigenvalues linked to the low-order modes. A large number of eigenvalues corresponding to the evanescent modes are on the line $x=1 / 2$ and do not cluster like for the sound-soft case. This behaviour penalizes the convergence rate of the GMRES. Similar conclusions arise for the CFIE (Figure 10(c)]. This is due to the fact that these three integral equations are first-kind Fredholm integral equations and so are illconditioned. In particular, the convergence rate of a Krylov iterative solver 


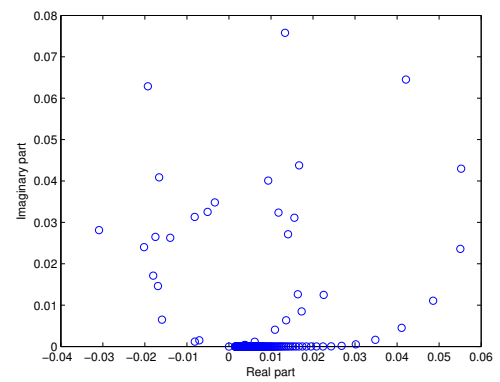

(a) Sound-soft: EFIE

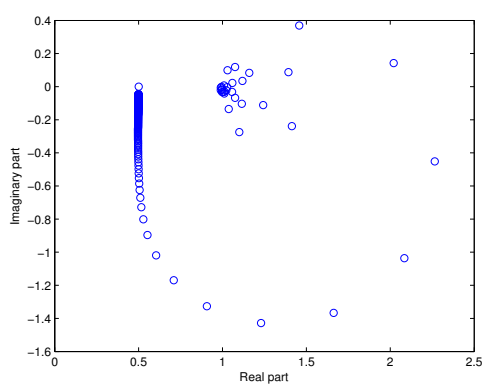

(b) Sound-soft: BW

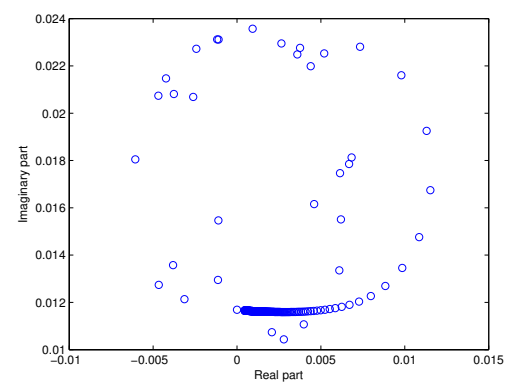

(c) Sound-soft: CFIE

Fig. 9 Sound-soft sphere: distribution of the eigenvalues for the EFIE, BW and CFIE.

will depend on the wavenumber $k$ as well as the mesh density. Using some asymptotics of the spherical Bessel and Hankel functions and some formal replacements, approximations of the eigenvalues can be obtained for these integral equations (see Section 8.2). For more general two-dimensional domains (convex, non-convex, polygon, starlike polygon, trapping domains) in the case of the acoustic sound-soft scattering problem, we refer for instance to [50,51] which study the conditioning and spectral properties of the usual boundary integral equations.

From this analysis, we clearly see that some efforts on analytical (or algebraic) preconditioning must be directed towards the EFIE for the sound-soft problem and EFIE, BW and CFIE for the sound-hard case.

\section{A first direction to improve the convergence: algebraic/analytical preconditioning techniques for the EFIE}

As seen above, the EFIE has a condition number which depends on both the wavenumber $k$ and the density of discretization points per wavelength $n_{\lambda}$ for both the sound-soft and sound-hard scattering problems. Furthermore, the 


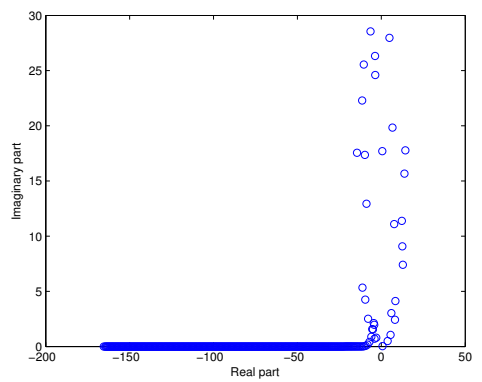

(a) Sound-hard: EFIE

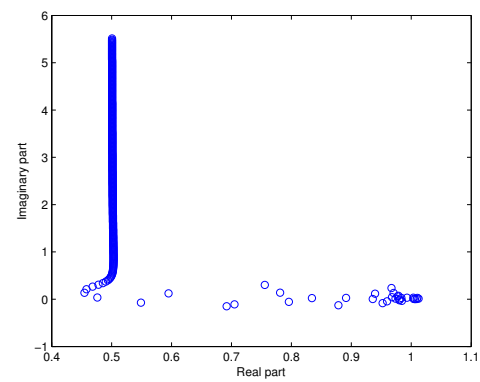

(b) Sound-hard: BW

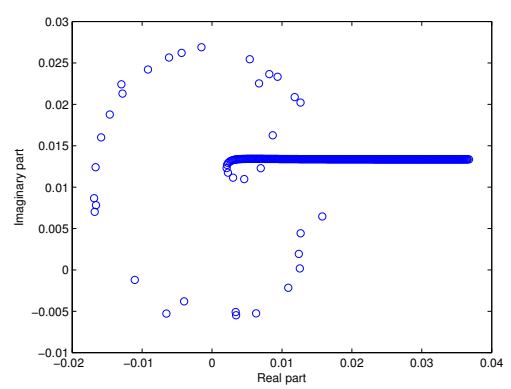

(c) Sound-hard: CFIE

Fig. 10 Sound-hard spheres: distribution of the eigenvalues for the EFIE, BW and CFIE.

eigenvalues spread out in the complex plane which is very penalizing in view of an iterative Krylov solution. One way to improve the convergence properties of the EFIE is to precondition it. Algebraic preconditioners have been proposed over the years but may fail, most particularly for large wavenumbers. Furthermore, they need to handle the full matrix which is incompatible with the idea of a matrix-free solver imposed by the FMM method. We propose here two possible directions for building analytical preconditioners: the first one uses the Calderón integral relations and the second one elements from the pseudodifferential operator theory.

\subsection{Integral operator preconditioning}

The idea of integral operator preconditioning uses the following Calderón relations [100]

$$
-\mathcal{L S}=\frac{\mathcal{I}}{4}-\mathcal{N}^{2}, \quad-\mathcal{S} \mathcal{L}=\frac{\mathcal{I}}{4}-\left(\mathcal{N}^{T}\right)^{2} .
$$

From these two equations, we can see that, since $\mathcal{N}$ is a compact operator, then $\mathcal{N}^{2}$ and $\left(\mathcal{N}^{T}\right)^{2}$ are compact and their eigenvalues tend towards zero. This 
also means that the eigenvalues of $-\mathcal{L S}$ and $-\mathcal{S L}$ cluster around $(1 / 4,0)$ in the complex plane since they are equal to the operator $\mathcal{I} / 4$ up to a compact perturbation. As a consequence, $-\mathcal{L}$ (respectively $-\mathcal{S}$ ) is a pseudo inverse operator of $\mathcal{S}$ (respectively $-\mathcal{L}$ ) and can serve as a preconditioner. This is an interesting property since the application of this preconditioner in a FMM environment only involves some evaluations of standard integral operators. This first application of Calderòn relations to preconditioning has been developed by Steinbach and Wendland in [110. It has been next applied to the EFIE for acoustics and electromagnetism by Christiansen and Nédélec in 52 . Extensions and other studies related to this approach are available in [13, 14, 15.

To show the improvement induced by a preconditioner based on Calderón relations, we consider the transmission scattering problem which consists in solving an exterior Helmholtz problem with wavenumber $k_{2}$ coupled with an interior Helmholtz problem for a wavenumber $k_{2} \sqrt{N}$ with transmission boundary conditions. This physically corresponds to scattering by a penetrable homogeneous isotropic scatterer. Following [15], we propose a Calderón preconditioner (denoted by $C$ ) for the two-field integral equation solution to the transmission problem (see [15] for more details). We consider on Figure 11(a) the convergence history of GMRES(50) for the preconditioned linear system in the case of the unit square cylinder (centered at the origin and with sidelength 2 ). We represent the dependence of the preconditioned algorithm according to both the wavenumber $k_{2}$ and the density $n_{\lambda}$ of discretization points per wavelength. The index $\sqrt{N}$ for the interior problem is $\sqrt{N}=2+i$. We can observe that the convergence is independent of the density $n_{\lambda}$ since it can be shown that the preconditioned integral equation is Fredholm second-kind. However, the convergence depends moderately on $k_{2}$, which is one of the limitations for Calderón preconditioners. Another example is given by the scattering problem by a penetrable kite-shaped object (see Figure 11(b) for $\sqrt{N}=1.55+0.64 i$. The same conclusions can be made from Figure 11(c). We can see on Figure $11(\mathrm{~d})$ (for $k_{2}=40$ and $n_{\lambda}=10$ ) that even if some eigenvalues spread out in the complex plane and imply a $k_{2}$-dependence of the iterative solver, a large cluster of eigenvalues characterizes the preconditioned matrix and results in the $n_{\lambda}$ independence. This first example illustrates the interest of considering the point of view of analytical preconditioning for solving scattering problems. Let us finally mention that examples in [15] show that ILU preconditioners fail in general and lead to a breakdown of the GMRES.

\subsection{Pseudodifferential operator preconditioning}

A second approach for preconditioning the EFIE uses the following result which precises the principal symbol of the single-layer operator $\mathcal{L}$ and normal derivative trace $\mathcal{S}$ of the double-layer potential.

Proposition 8 Let $\mathcal{L}$ and $\mathcal{S}$ be the single-layer and normal derivative trace of the double-layer potentials defined by the expressions (24), respectively. Let 


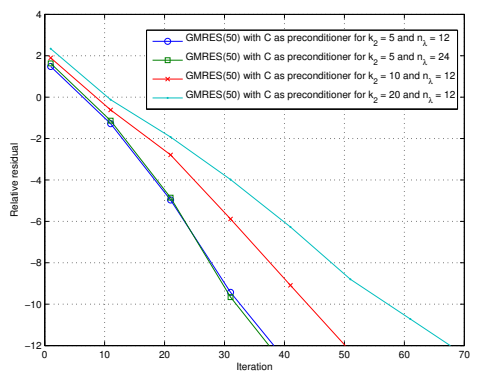

(a) Convergence history of GMRES(50) for the unit square cylinder.

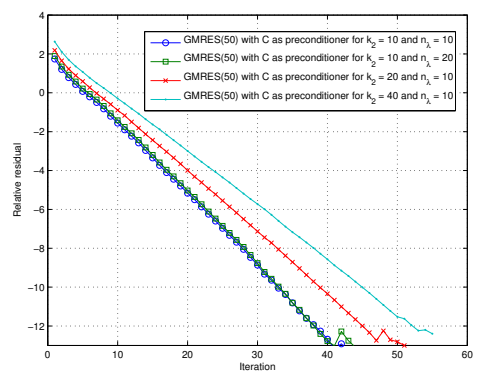

(c) Convergence history of GMRES(50) for the kite-shaped scatterer.

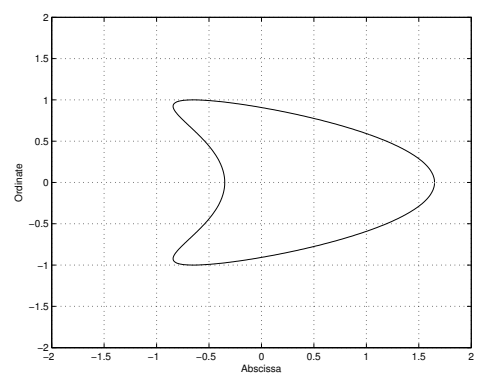

(b) The kite-shaped scatterer.

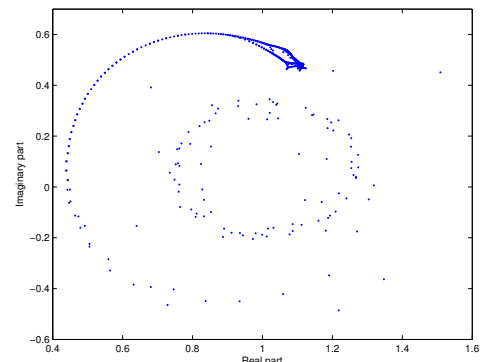

(d) Eigenvalue distribution.

Fig. 11 Calderón preconditioner for transmission problems.

$\boldsymbol{\xi}$ be the dual variable of $\boldsymbol{x}$ by Fourier transform for $\boldsymbol{x}$ restricted to $\Gamma$. Then, the principal symbols of $\mathcal{L}$ and $\mathcal{S}$, denoted by $\sigma_{p}(\mathcal{L})$ and $\sigma_{p}(\mathcal{S})$, are given by

$$
\sigma_{p}(\mathcal{L})=\frac{i}{2 \sqrt{k^{2}-|\boldsymbol{\xi}|^{2}}} \quad \text { and } \quad \sigma_{p}(\mathcal{S})=-\frac{\sqrt{k^{2}-|\boldsymbol{\xi}|^{2}}}{2 i} .
$$

Following an approach similar to the one developed in Section 3.3 but for integral equations, a good preconditioner for the sound-soft scattering problem based on the EFIE representation is $O p\left(\sigma_{p}(\mathcal{L})^{-1}\right)=-4 O p\left(\sigma_{p}(\mathcal{S})\right)$ since then

$$
-O p\left(\sigma_{p}(\mathcal{S})\right) \mathcal{L}=\frac{\mathcal{I}}{4}+R
$$

where $R$ is a pseudodifferential operator of order less or equal to -1 . One of the crucial points in this approach is that $k$ is also considered as a symbol (associated with the time derivative operator $\partial_{t}$ if we come back to a wave equation) of order 1 . Then, the symbol $\sigma_{p}(\mathcal{S})$ is homogeneous of order 1 and defines a corresponding classical pseudodifferential operator of order 1 . We see here that the equation 45 is typically a pseudodifferential operator version 
of the second Calderón relation (43). The main difference is that the operator $O p\left(\sigma_{p}(\mathcal{S})\right)$ is a correct approximation of the inverse of $\mathcal{L}$ in both its hyperbolic and elliptic zones. This is not the case of $\mathcal{S}$ which is only a good approximation of the inverse in the elliptic part since the error term $-\left(\mathcal{N}^{T}\right)^{2}$ is compact (for large values of $|\boldsymbol{\xi}|$ compared to $k$ ). Consequently, the convergence rate of the iterative Krylov solver is independent of the mesh refinement parameter $n_{\lambda}$ and the dependence according to $k$ is weakened. For the sound-hard scattering problem, a suitable choice is $-4 O p\left(\sigma_{p}(\mathcal{L})\right)$ for preconditioning $\mathcal{S}$ and the same conclusions arise.

Concerning the implementation in a FMM code, including the operator $O p\left(\sigma_{p}(\mathcal{S})\right)$ can be made independently of the integral equations. For effectiveness, a complex Padé approximation of the square-root must be considered (see Section 8.2 , resulting in the numerical solution of a coupled system of surface dissipative Helmholtz equations on $\Gamma$. Their approximate solution can be considered by using an ILU preconditioner and an iterative scheme at the cost $\mathcal{O}\left(N_{V}\right)$, which is much less than applying an integral operator. We refer to 14 for the interested reader where much more details about the implementation are given. Some partial elements are also developed in Section 8.3

To show the robustness of such an approach, we report on Figures 12 the number of MVPs required for solving the sound-hard scattering problem by a strip of length 2 with GMRES(50) for a tolerance $\varepsilon=10^{-6}$. We use the EFIE and represent the number of MVPs with respect to the density $n_{\lambda}$ (for $k=15$, figure 12(a) and the wavenumber $k$ (for $n_{\lambda}=20$, figure 12(b)). The EFIE is referred to as "DBIE" here, the pseudodifferential preconditioner based on $-4 O p\left(\sigma_{p}(\mathcal{L})\right)$ and a Padé approximation by "Padé-type", the Calderón integral preconditioner $[\mathcal{L}]$ by "Calderon". Furthermore, an algebraic SPAI (SParse Approximate Inverse) preconditioner with sparsity level $N_{v}=5$ is also used for comparison. The choice $N_{v}=5$ makes the preconditioning matrix already quite dense (about $20 \%$ of nonzero coefficients). Another drawback is that it is built on the full initial EFIE matrix $[\mathcal{S}]$ which is not available when considering a FMM solution. On this simple example, we can see that the number of iterations is independent of both $n_{\lambda}$ and $k$. We report on Figure 12(c) the convergence history for all methods. We can see the superiority here of the two analytical preconditioners over the algebraic SPAI. A second example is given on Figure 12(d) for a cobra-like shaped scatterer which is an open curved resonator modeling an inlet. We can again observe the very good behaviour of the iterative solvers with analytical preconditioners.

In references 14, other numerical examples show that some problems can arise for the Calderón preconditioner if the scatterer is composed of two parallel strips or screens due to the existence of resonances. It seems that, for this kind of situation, the pseudodifferential preconditioners are more robust. However, for a single three-dimensional open surface, some problems are still open like for instance how to correctly handle the edges effects in a pseudodifferential approach. In the case of Calderón operators, this point is quite naturally treated. A hybrid Calderón-pseudodifferential preconditioner is proposed in [58. 


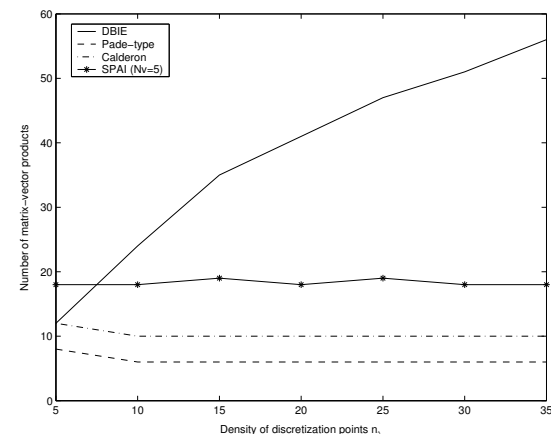

(a) MVPs vs. $n_{\lambda}$

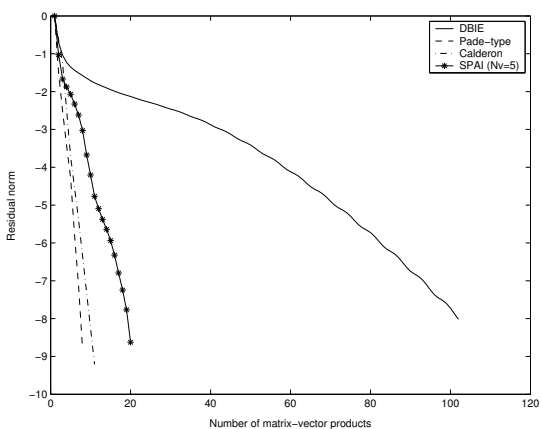

(c) Convergence history of GMRES(50) for the strip.

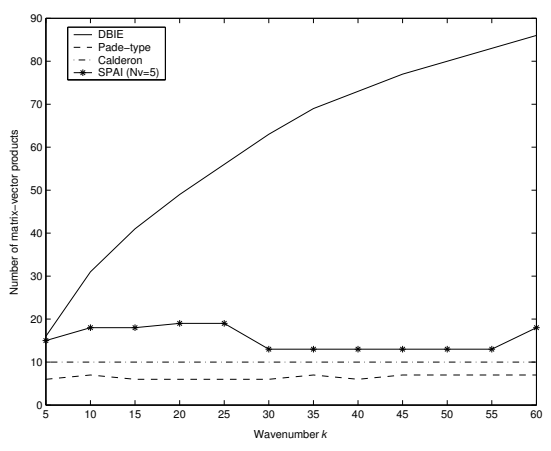

(b) MVPs vs. $k$.

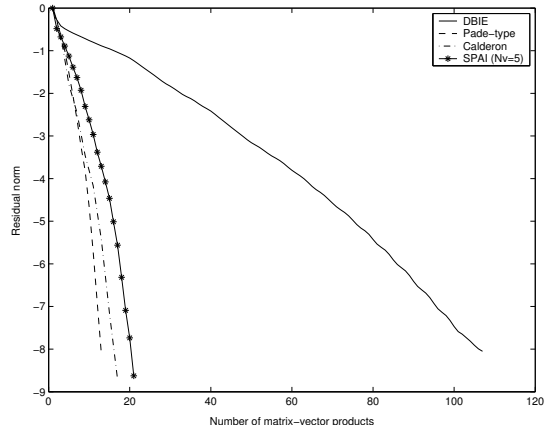

(d) Convergence history of GMRES(50) for the cobra-like scatterer.

Fig. 12 Pseudodifferential preconditioner for scattering problems.

\section{A second direction to improve the convergence: generalized Combined Field Integral Equations in acoustic}

We restrict our presentation to a Neumann boundary condition. Indeed, we have seen in Section 6 that the standard combined field integral equations (both BW and CFIE) are second-kind Fredholm integral equations for a soundsoft obstacle. As a consequence, the convergence rate of an iterative solver applied to these equations is independent of the density $n_{\lambda}$ but still depends slightly on $k$. In the same spirit as what is proposed below, this dependence can be weakened by developing generalized (direct and indirect) combined field integral equations. In the case of the sound-hard obstacle, much work is required since the standard integral equations are first-kind Fredholm integral equations. Furthermore, our construction of new second-kind integral equations preserves the well-posedness of these new integral equations, meaning that they are all free of any spurious resonance. 
8.1 Construction of generalized combined field integral equations

The starting point of the method proposed in [16, 17, 58 is based on the exterior Helmholtz integral formulation

$$
u^{+}=D \gamma_{0}^{+} u^{+}-L \gamma_{1}^{+} u^{+}, \text {in } \Omega^{+} .
$$

Consider the exact exterior Neumann-to-Dirichlet (called NtD) map $V^{\text {ex }}$

$$
\begin{aligned}
V^{\mathrm{ex}}: H^{-1 / 2}(\Gamma) & \rightarrow H^{1 / 2}(\Gamma) \\
\gamma_{1}^{+} u^{+} & \mapsto \gamma_{0}^{+} u^{+}=V^{\mathrm{ex}} \gamma_{1}^{+} u^{+} .
\end{aligned}
$$

The NtD operator $V^{\mathrm{ex}}$ is a nonlocal pseudodifferential operator of order -1 . We write the integral representation (46) under the form

$$
u^{+}=D V^{\mathrm{ex}} \gamma_{1}^{+} u^{+}-L \gamma_{1}^{+} u^{+}, \text {in } \Omega^{+} .
$$

Applying the exterior normal derivative trace to (48), we get thanks to Proposition 5

$$
\gamma_{1}^{+} u^{+}=\left(\mathcal{S} V^{\mathrm{ex}}+\frac{\mathcal{I}}{2}-\mathcal{N}\right) \gamma_{1}^{+} u^{+}, \text {in } H^{1 / 2}(\Gamma),
$$

and hence the exact NtD operator $V^{\text {ex }}$ satisfies on $\Gamma$

$$
\frac{\mathcal{I}}{2}-\mathcal{N}+\mathcal{S} V^{\mathrm{ex}}=\mathcal{I}
$$

Thus we conclude that plugging the exact NtD operator $V^{\mathrm{ex}}$ (instead of the constant $\eta$, see (41) ) into the integral formulation leads to the identity operator which can be solved trivially in one iteration (direct solver). In this sense, the exact $\mathrm{NtD}$ is an ideal regularizing operator for the operator $\mathcal{S}$. Furthermore, assuming that $k$ is not an eigenfrequency of the Helmholtz equation in $\Omega^{-}$ with a Neumann homogeneous boundary condition, we deduce from (50) that the NtD map is expressed in terms of elementary boundary integral operators on $\Gamma$ by

$$
V^{\mathrm{ex}}=\mathcal{S}^{-1}\left(\frac{\mathcal{I}}{2}+\mathcal{N}\right)
$$

The first Calderón formula (see (43)) leads to another integral representation

$$
V^{\mathrm{ex}}=-\left(\frac{\mathcal{I}}{2}+\mathcal{N}\right)^{-1} \mathcal{L}
$$

However, it is too expensive numerically to apply one of these representations of the operator $V^{\text {ex }}$ as a regularizing operator for $\mathcal{S}$.

Instead, an approximation $\tilde{V}$ of the exact $\mathrm{NtD}$ operator $V^{\mathrm{ex}}$ is introduced such that

$$
\varphi=\tilde{V} \psi,
$$

with $(\varphi, \psi)$ an approximation of the Cauchy data $\left(\gamma_{0}^{+} u^{+}, \gamma_{1}^{+} u^{+}\right)$. We propose to solve the following integral equation

$$
\left(\frac{\mathcal{I}}{2}-\mathcal{N}+\mathcal{S} \tilde{V}\right) \psi=-\gamma_{1}^{+} u^{\text {inc }}, \text { in } H^{1 / 2}(\Gamma)
$$


based on the exterior integral representation

$$
u^{+}=-L \psi+D \varphi, \text { in } \Omega^{+} .
$$

The spectral properties of $(53)$ depend on the approximate NtD map $\tilde{V}$. Let us consider the low-order approximation $\tilde{V}=-i / k$ (corresponding to the Sommerfeld radiation condition). This choice leads to the standard BW integral equation (41) with the optimal parameter of Kress [87. Consequently, the integral equation $(53)$ can be seen as a generalization of the standard BW integral equation. A similar approach can also be developed for constructing a generalized CFIE: for $\beta \in] 0,1[$

$$
\left((1-\beta)\left(-\frac{\mathcal{I}}{2}+\mathcal{D}\right)-\beta \tilde{V} \mathcal{S}\right) \phi=-\left((1-\beta) \gamma_{0}^{+} u^{+}+\beta \tilde{V} \gamma_{1}^{+} u^{\text {inc }}\right), \text { in } H^{1 / 2}(\Gamma)
$$

by composition of the operator $\mathcal{S}$ (EFIE) with $-\tilde{V}$ and then adding the contribution of the MFIE by a suitable linear combination. The pseudodifferential operator $\tilde{V}$ plays the role of a regularizing operator for the first-order operator $\mathcal{S}$. It should therefore be of order -1 . This is not the case by considering the low-order approximation $\tilde{V}=-i / k$ which is of order zero. For this reason, high-order approximations of the NtD operator are needed. This can be done for example by adapting techniques related to the Beam Propagation Method (BPM) [75] or On-Surface Radiation Conditions (OSRCs) methods [10,11, 12, 18, 78, 90. In particular, we refer to [11] which gives an overview of OSRC approaches. From [18, an accurate approximation of the NtD map is expressed by the square-root operator

$$
\tilde{V}=\frac{1}{i k}\left(1+\frac{\Delta_{\Gamma}}{k_{\delta}^{2}}\right)^{-1 / 2}
$$

which is the principal part of the boundary integral operator $-2 \mathcal{L}$ (see 52 ) and Proposition 8. The operator $\Delta_{\Gamma}$ is the usual Laplace-Beltrami operator over the surface $\Gamma$ (see also subsection 9.1) and the parameter $k_{\delta}=k+i \delta$ is a complexified wavenumber. The aim of the damping parameter $\delta>0$ is to regularize the square-root operator in the transition zone of grazing rays [18]. An optimal value of this parameter, related to the mean curvature of $\Gamma$ and to $k$, is given in [18. Incorporating the operator $\tilde{V}$, the generalized combined integral equations 53 and (55) are uniquely solvable [58 for any frequency $k>0$ and damping parameter $\delta>0$. Moreover, they are second-kind Fredholm integral equations unlike the standard formulations (53) and (39). This is an interesting property for an iterative Krylov solution.

8.2 The special case of the unit sphere

The eigenvalues of the operator $-\Delta_{\Gamma}$ are expressed by $\mu_{m}=m(m+1)$. A direct computation gives the eigenvalues $\mathcal{B}_{m}^{N, \delta}$ and $\mathcal{C}_{m}^{N, \delta}$ of respectively the 


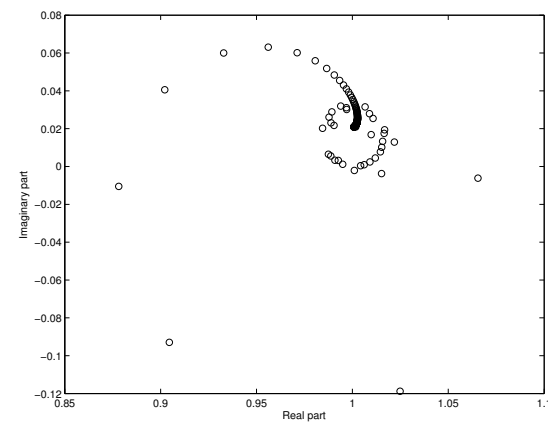

(a) Generalized BW

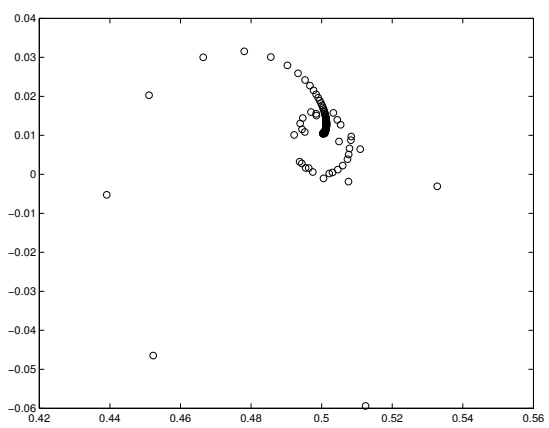

(b) Generalized CFIE

Fig. 13 Sound-hard sphere: distribution of the eigenvalues for the generalized combined field integral equations.

generalized BW and the CFIE operators as

$$
\mathcal{B}_{m}^{N, \delta}=-i k^{2}\left(h_{m}^{(1)}\right)^{\prime}(k)\left[i j_{m}^{\prime}(k)\left(1-\frac{\mu_{m}}{k_{\delta}^{2}}\right)^{-1 / 2}+j_{m}(k)\right],
$$

and

$$
\mathcal{C}_{m}^{N, \delta}=k^{2}\left(h_{m}^{(1)}\right)^{\prime}(k)\left[-i(1-\beta) j_{m}(k)+\beta\left(1-\frac{\mu_{m}}{k_{\delta}^{2}}\right)^{-1 / 2} j_{m}^{\prime}(k)\right] .
$$

We report on Fig. 13 the eigenvalues of the generalized integral operators by taking $k=30$ and $m_{\max }=12 k$ modes. The eigenvalues of the generalized BW and CFIE operators cluster around the points $(1,0)$ and $(1 / 2,0)$, respectively. This observation is partially related to the property that these integral equations are Fredholm second-kind but also to the fact that an accurate approximation of the $\mathrm{NtD}$ operator is injected. By using suitable asymptotic expansions of the Bessel and Hankel functions [1, we justify this remark by the following approximations of the eigenvalues [58]

- in the elliptic zone (evanescent modes) for large values of $m$

$$
\left.\mathcal{B}_{m}^{N, \delta}=\frac{1}{2}+\frac{k_{\delta}}{2 k}+\mathcal{O}\left(\frac{1}{m^{3}}\right) \text {, and } \mathcal{C}_{m}^{N, \delta}=\frac{1}{2}+\beta\left(\frac{k}{2 k_{\delta}}-\frac{1}{2}\right)+\mathcal{O}\left(\frac{1}{m^{3}}\right), \beta \in\right] 0,1[.
$$

- in the hyperbolic zone (propagative modes) for large wavenumbers $k$

$$
\mathcal{B}_{m}^{N, \delta}=1+\mathcal{O}\left(\frac{1}{k}\right) \text {, and } \mathcal{C}_{m}^{N, \delta}=\frac{1}{2}+\mathcal{O}\left(\frac{1}{k}\right)(\text { for } \beta=0.5) .
$$

The coupling between low-(propagative) and high-order (evanescent) modes is observable as a loop around the accumulation points $(1,0)$ and $(1 / 2,0)$ for the generalized BW and CFIE, respectively. From these observations, we can expect the fast convergence of a Krylov solver for computing the solution of 53 or 55 . 
8.3 Numerical issues: implementation and examples

Let us denote by $[P]$ the matrix associated with the linear discretization of a given integral operator $P$. The discretization of (53) leads to the $N_{V} \times N_{V}$ dense complex linear system

$$
\left(\frac{[\mathcal{I}]}{2}-[\mathcal{N}]+[\mathcal{S}][\tilde{V}]\right) \boldsymbol{\psi}_{\boldsymbol{h}}=\boldsymbol{g}_{\boldsymbol{h}}
$$

The complex valued vectors $\boldsymbol{\psi}_{\boldsymbol{h}}$ and $\boldsymbol{g}_{\boldsymbol{h}}$ of $\mathbb{C}^{N_{V}}$ are respectively the $\mathbb{P}_{1}$ interpolated unknown density $\psi$ and the right-hand side of $(53)$. The matrix representation $[\mathcal{I}]$ of the identity operator $\mathcal{I}$ is the surface mass matrix. We refer for example to 962 for the direct approximation of the integral operators $\mathcal{N}$ and $\mathcal{S}$. Of course, a FMM implementation can also be derived in the background of Galerkin methods for a fast evaluation of MVP. Concerning the discretization of the pseudodifferential operator $\tilde{V}$, rational Padé approximants are applied for an efficient local representation of the square-root operator. In [18, the authors use the rotating branch-cut technique, introduced by Milinazzo al. 98], which consists in using the rational approximation defined by

$$
\sqrt{1+X} \approx e^{i \theta_{p} / 2} R_{N_{p}}\left((1+X) e^{-i \theta_{p}}-1\right)=C_{0}+\sum_{j=1}^{N_{p}} \frac{A_{j} X}{1+B_{j} X}
$$

where $\theta_{p}$ is the rotation angle of the usual branch-cut $\{z \in \mathbb{R}, z<-1\}$ of the square-root operator, and $R_{N_{p}}$ denotes the standard real-valued Padé approximation of order $N_{p}$. The complex-valued coefficients $C_{0},\left(A_{j}, B_{j}\right)_{j=1, \cdots, N_{p}}$ are related to the real Padé coefficients and the angle $\theta_{p}[18$. Since the square-root operator is implemented in an iterative solver, we must be able to compute $\mathbf{y}=[\tilde{V}] \mathbf{x}$ efficiently, for a given vector $\mathbf{x} \in \mathbb{C}^{N_{V}}$. This can be achieved by writing

$$
\left(1+\frac{\Delta_{\Gamma}}{k_{\delta}^{2}}\right) \mathbf{y}=\frac{1}{i k}\left(1+\frac{\Delta_{\Gamma}}{k_{\delta}^{2}}\right)^{1 / 2} \mathbf{x}
$$

Then, the MVP $\mathbf{y}=[\tilde{V}] \mathbf{x}$ is computed by first solving $N_{p}$ uncoupled dissipative surface Helmholtz equations

$$
\left(\frac{B_{j}}{k_{\delta}^{2}}\left[\Delta_{\Gamma}\right]+[\mathcal{I}]\right) \mathbf{x}_{j}=[\mathcal{I}] \mathbf{x}, \quad j=1, \ldots, N_{p},
$$

using a variational formulation and a BEM, and $\mathbf{y}$ is then solution to

$$
\left([\mathcal{I}]+\frac{\left[\Delta_{\Gamma}\right]}{k_{\delta}^{2}}\right) \mathbf{y}=\frac{1}{i k}\left(C_{0}[\mathcal{I}] \mathbf{x}+\sum_{j=1}^{N_{p}} \frac{A_{j}}{k_{\delta}^{2}}\left[\Delta_{\Gamma}\right] \mathbf{x}_{j}\right) .
$$

The matrix $\left[\Delta_{\Gamma}\right]$ is the surface stiffness matrix on $\Gamma_{h}$. Let us mention that the solution of the $\left(N_{p}+1\right)$ linear systems $(59)$ and $(60)$ is computed by an ILUT preconditioned GMRES and only requires 2 or 3 iterations to get the solution [58]. Therefore, applying the square-root operator results in a cost 

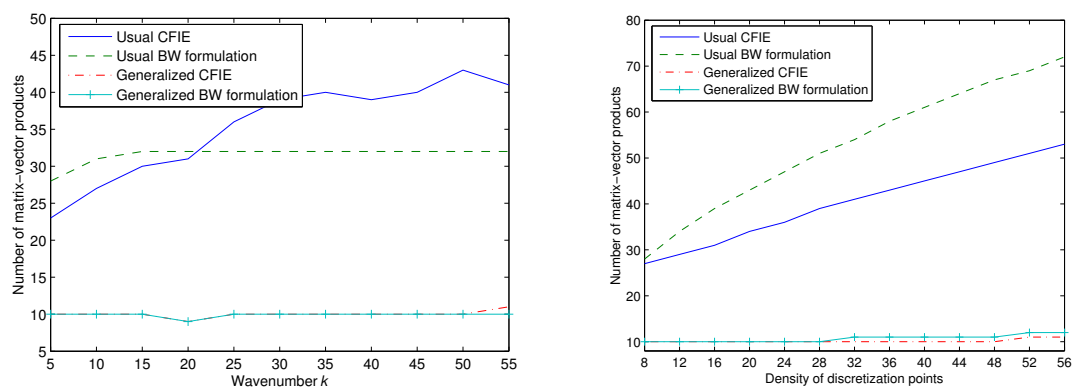

Fig. 14 Sound-hard unit square cylinder: number of MVPs for the four integral formulations with respect to the wavenumber $k$ (left: $n_{\lambda}=10$ ) and the density $n_{\lambda}$ of discretization points per wavelength (right: $k=10$ )

of the order $\mathcal{O}\left(N_{V}\right)$ which is much less than applying an elementary integral operator (even with a FMM implementation).

We present now some numerical results to compare the efficiency of the GMRES without restart and for a tolerance equal to $\varepsilon=10^{-6}$ when using the standard and generalized BW and CFIE. We report on Figure 14 an example of computation for the scattering of an incident plane wave of incidence 45 degrees by the unit square cylinder. For the usual formulations, we can see that the number of MVPs grows linearly according to the density $n_{\lambda}$. Indeed, these two formulations are of the first-kind. Furthermore, the number of iterations also depends on $k$ (see the next example for the standard BW formulation). The problem is related to the fact that injecting the Sommerfeld approximation is not enough to handle the $k$-dependence of the convergence. These two points can be improved by considering the generalized formulations. The conclusions are confirmed for the three-dimensional case. In Figure 15, we present the case of the scattering of an incident plane wave of incidence zero degree by an ellipsoidal scatterer of semi-axis $1,0.5$ and 0.5 along the $x_{1^{-}}, x_{2^{-}}$and $x_{3^{-}}$ directions, respectively, and centered at the origin. Here again, the convergence of the GMRES is independent of both the parameters $k$ and $n_{\lambda}$ while this is not the case for the two standard formulations.

To end, we consider a more realistic nonconvex 3D example: the geometry is a submarine (see Figure 16) characterized by a very large length and a sharp and irregular shape at the back. The dimensions are: length $=43 \mathrm{~m}$, thickness $\approx 4$ to $7 \mathrm{~m}$, high $\approx 4$ to $7 \mathrm{~m}$. Figure 17 shows the eigenvalue distribution of the standard and the generalized CFIE operators. An excellent eigenvalue clustering around $(1,0)$ is observed with the generalized CFIE. This implies a fast convergence of GMRES independently of both a frequency increase and mesh refinement (see Fig. 18). These results come from [60] in which a singlelevel FMM and the generalized CFIE are efficiently coupled and validated for several 3D tests. 

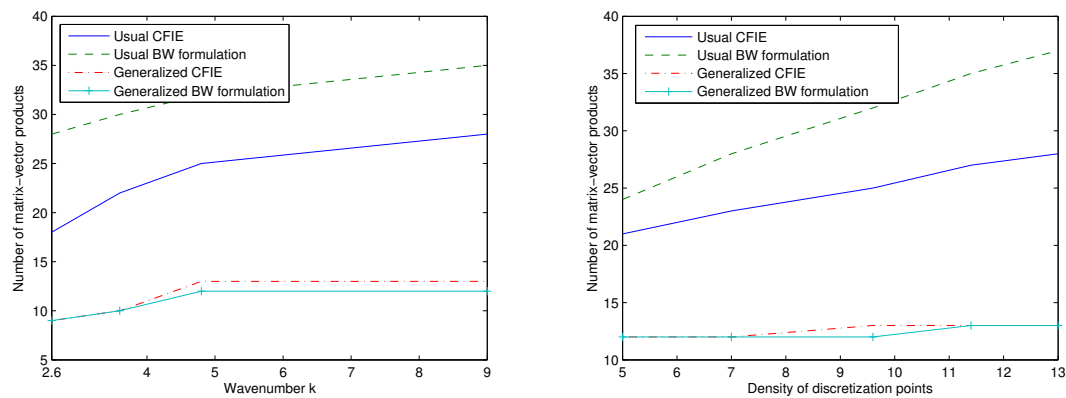

Fig. 15 Sound-hard ellipsoidal scatterer: number of MVPs for the four integral formulations with respect to the wavenumber $k$ (left: $n_{\lambda}=10$ ) and the density $n_{\lambda}$ of discretization points per wavelength (right: $k=5$ ).

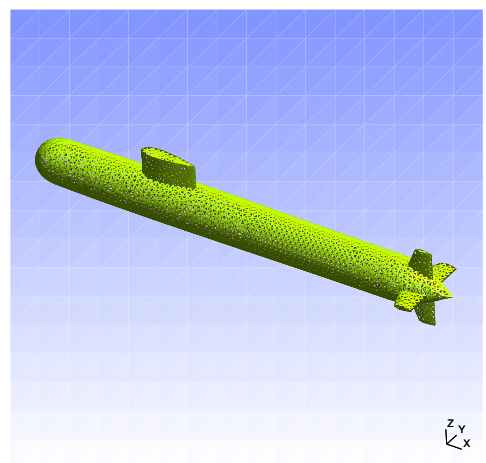

Fig. 16 A 3D realistic configuration: mesh of a submarine.

\section{Generalized Combined Field Integral Equations in electromagnetism}

The aim of this section is to explain how to extend the previous generalized BW/CFIE to the Maxwell's equations for electromagnetic waves.

9.1 The Maxwell exterior problem and standard boundary integral equations

The three-dimensional scatterer is denoted by $\Omega^{-}$, with a regular compact boundary $\Gamma=\partial \Omega^{-}$. It represents a perfectly conducting object. The exterior domain is defined by $\Omega^{+}=\mathbb{R}^{3} \backslash \overline{\Omega^{-}}$. We assume that $\Omega^{-}$is illuminated by an incident time-harmonic electromagnetic wave $\left(\boldsymbol{E}^{\text {inc }}, \boldsymbol{H}^{\text {inc }}\right)$, with timedependence $e^{-i \omega t}, \omega$ being the field pulsation. The incident field interacts with $\Omega^{-}$and creates a scattered field $\left(\boldsymbol{E}^{+}, \boldsymbol{H}^{+}\right)$. The total electromagnetic 

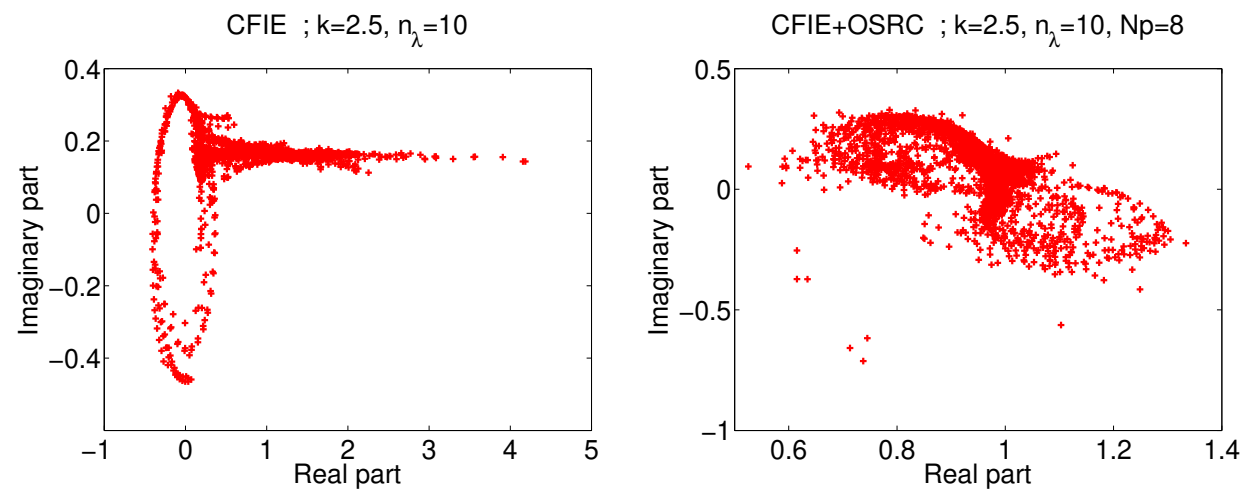

Fig. 17 Sound-hard submarine: distribution of the eigenvalues (left: standard CFIE, right: generalized CFIE), $k=2.5, n_{\lambda}=10$.
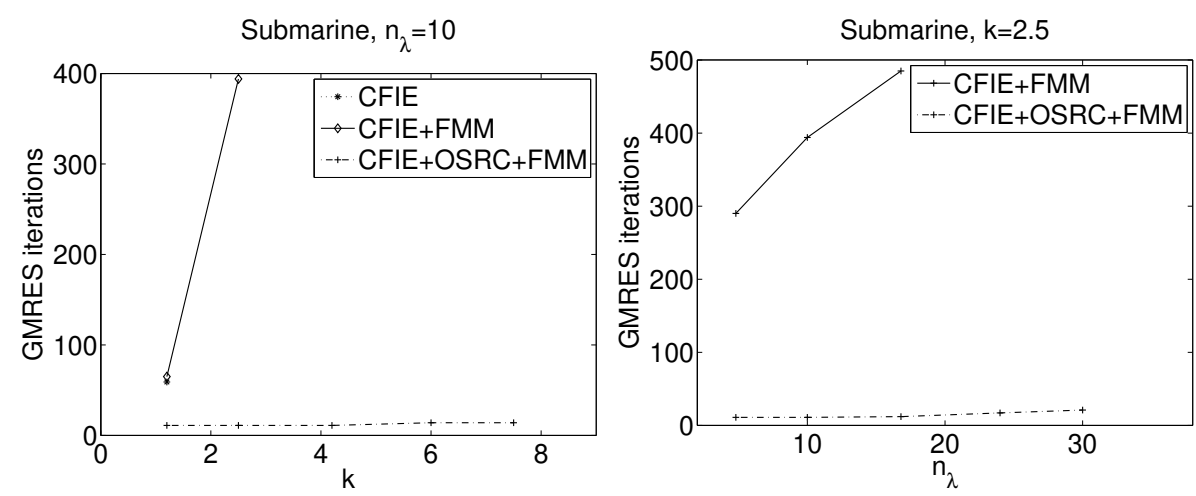

Fig. 18 Sound-hard submarine: number of GMRES iterations with respect to the wavenumber $k$ (left) and the density $n_{\lambda}$ (right).

field $(\boldsymbol{E}, \boldsymbol{H})$ is given by

$$
\left\{\begin{array}{l}
\boldsymbol{E}=\boldsymbol{E}^{+}+\boldsymbol{E}^{\text {inc }} \text { in } \mathbb{R}^{3} \backslash \Omega^{-}, \\
\boldsymbol{H}=\boldsymbol{H}^{+}+\boldsymbol{H}^{\text {inc }} \text { in } \mathbb{R}^{3} \backslash \Omega^{-},
\end{array}\right.
$$

where $\boldsymbol{E}$ and $\boldsymbol{H}$ are the electric and magnetic fields, respectively. The exterior Maxwell problem consists in computing $\left(\boldsymbol{E}^{+}, \boldsymbol{H}^{+}\right)$solution to the exterior time-harmonic Maxwell system [56, 99, 100]

$$
\begin{cases}\operatorname{curl} \boldsymbol{E}^{+}-i k Z_{0} \boldsymbol{H}^{+}=0 & \text { in } \Omega^{+}, \\ \operatorname{curl} \boldsymbol{H}^{+}+i k Z_{0}^{-1} \boldsymbol{E}^{+}=0 & \text { in } \Omega^{+},\end{cases}
$$

with the perfectly conducting boundary condition

$$
\boldsymbol{n} \times\left(\boldsymbol{E}_{\mid \Gamma}^{+} \times \boldsymbol{n}\right)=-\boldsymbol{n} \times\left(\boldsymbol{E}_{\mid \Gamma}^{\text {inc }} \times \boldsymbol{n}\right), \quad \text { on } \Gamma .
$$


The wavenumber is $k=\omega / c$, with $c$ the speed of light, $\boldsymbol{n}$ is the outwardly directed unit normal vector to $\Omega^{-}$and $Z_{0}$ is the impedance of the vacuum. The operator curl is the standard rotational operator of a three-dimensional complex-valued vector field and $\times$ denotes the vector cross-product of two vector fields in 3D. In addition, to get the uniqueness of the solution to the exterior problem [99,100], $\left(\boldsymbol{E}^{+}, \boldsymbol{H}^{+}\right)$must satisfy the Silver-Müller radiation condition at infinity

$$
\lim _{\|\boldsymbol{x}\| \rightarrow+\infty}\|\boldsymbol{x}\|\left(\boldsymbol{E}^{+}+Z_{0} \frac{\boldsymbol{x}}{\|\boldsymbol{x}\|} \times \boldsymbol{H}^{+}\right)=0
$$

The existence and uniqueness of $\left(\boldsymbol{E}^{+}, \boldsymbol{H}^{+}\right)$, solution to $62-(64)$ can be proved [100] in suitable Sobolev spaces, for any wavenumber $k>0$ and incident field $\boldsymbol{E}^{\text {inc }}$.

One possible way to solve $62-(64)$ is to use an integral representation of $\boldsymbol{E}^{+}$and $\boldsymbol{H}^{+}$based on suitable vector fields tangent to $\Gamma$. Let us start by giving the well-known Stratton-Chu formulae [56, 99, 100].

Theorem 8 The solution $\left(\boldsymbol{E}^{+}, \boldsymbol{H}^{+}\right)$to 62)- 64) has the following integral representation

$$
\begin{cases}\boldsymbol{E}^{+}(\boldsymbol{x})=i k Z_{0} \mathcal{T} \boldsymbol{J}(\boldsymbol{x})+\mathcal{K} \boldsymbol{M}(\boldsymbol{x}), & \boldsymbol{x} \in \Omega^{+} \\ \boldsymbol{H}^{+}(\boldsymbol{x})=-K \boldsymbol{J}(\boldsymbol{x})+i k Z_{0}^{-1} \mathcal{T} \boldsymbol{M}(\boldsymbol{x}), & \boldsymbol{x} \in \Omega^{+}\end{cases}
$$

In the above equations, $\boldsymbol{J}$ and $\boldsymbol{M}$ designate the electric and magnetic equivalent surface currents respectively given by

$$
\boldsymbol{J}(\boldsymbol{x})=\boldsymbol{n}(\boldsymbol{x}) \times \boldsymbol{H}_{\mid \Gamma}^{+}(\boldsymbol{x}) \text { and } \boldsymbol{M}(\boldsymbol{x})=-\boldsymbol{n}(\boldsymbol{x}) \times \boldsymbol{E}_{\mid \Gamma}^{+}(\boldsymbol{x}), \quad \boldsymbol{x} \in \Gamma .
$$

The subscripts \pm correspond to traces onto $\Gamma$ from $\Omega^{ \pm}$. The electric and magnetic potentials $\mathcal{T}$ and $\mathcal{K}$ are defined, for $\boldsymbol{x} \notin \Gamma$, by

$$
\begin{aligned}
\mathcal{T} \boldsymbol{J}(\boldsymbol{x}) & =\frac{1}{k^{2}} \operatorname{curl} \operatorname{curl} \int_{\Gamma} G(\boldsymbol{x}, \boldsymbol{y}) \boldsymbol{J}(\boldsymbol{y}) d \Gamma(\boldsymbol{y}) \\
& =\int_{\Gamma} G(\boldsymbol{x}, \boldsymbol{y}) \boldsymbol{J}(\boldsymbol{y}) d \Gamma(\boldsymbol{y})+\frac{1}{k^{2}} \nabla_{\Gamma} \int_{\Gamma} G(\boldsymbol{x}, \boldsymbol{y}) \operatorname{div}_{\Gamma} \boldsymbol{J}(\boldsymbol{y}) d \Gamma(\boldsymbol{y}) \\
\mathcal{K} \boldsymbol{M}(\boldsymbol{x}) & =-\operatorname{curl} \int_{\Gamma} G(\boldsymbol{x}, \boldsymbol{y}) \boldsymbol{M}(\boldsymbol{y}) d \Gamma(\boldsymbol{y}) \\
& =\int_{\Gamma} \nabla_{\boldsymbol{y}} G(\boldsymbol{x}, \boldsymbol{y}) \times \boldsymbol{M}(\boldsymbol{y}) d \Gamma(\boldsymbol{y}),
\end{aligned}
$$

where $G$ is the free-space Green's function given by (18).

The integral operators $\mathcal{T}$ and $\mathcal{K}$ are called the Maxwell single- and doublelayer potentials, and the surface currents $\boldsymbol{J}$ and $\boldsymbol{M}$ are the Cauchy data which represent the new unknowns for the scattering problem (62). The incident field $\left(\boldsymbol{E}^{\mathrm{inc}}, \boldsymbol{H}^{\mathrm{inc}}\right)$ is solution to the Maxwell's equations in $\mathbb{R}^{3}$ and does not satisfy 
the Silver-Müller radiation condition (64). The traces of incident electric and magnetic fields, i.e. $\boldsymbol{E}^{\text {inc }}$ and $\boldsymbol{H}^{\text {inc }}$, have no jump across $\Gamma$ and satisfy

$$
\begin{cases}\mathbf{0}=i k Z_{0} \mathcal{T} \boldsymbol{J}^{\mathrm{inc}}(\boldsymbol{x})+\mathcal{K} \boldsymbol{M}^{\mathrm{inc}}(\boldsymbol{x}), & \boldsymbol{x} \in \Omega^{+}, \\ \mathbf{0}=-\mathcal{K} \boldsymbol{J}^{\mathrm{inc}}(\boldsymbol{x})+i k Z_{0}^{-1} \mathcal{T} \boldsymbol{M}^{\mathrm{inc}}(\boldsymbol{x}), & \boldsymbol{x} \in \Omega^{+},\end{cases}
$$

with

$$
\begin{aligned}
& \boldsymbol{J}^{\text {inc }}(\boldsymbol{x})=\boldsymbol{n}(\boldsymbol{x}) \times \boldsymbol{H}_{\mid \Gamma}^{\mathrm{inc}}(\boldsymbol{x}), \quad \boldsymbol{x} \in \Gamma, \\
& \boldsymbol{M}^{\mathrm{inc}}(\boldsymbol{x})=-\boldsymbol{n}(\boldsymbol{x}) \times \boldsymbol{E}_{\mid \Gamma}^{\text {inc }}(\boldsymbol{x}), \boldsymbol{x} \in \Gamma .
\end{aligned}
$$

For the perfectly conducting case (63), we then obtain

$$
\begin{cases}\boldsymbol{E}^{+}(\boldsymbol{x})=i k Z_{0} \mathcal{T} \boldsymbol{J}(\boldsymbol{x}), & \boldsymbol{x} \in \Omega^{+}, \\ \boldsymbol{H}^{+}(\boldsymbol{x})=-\mathcal{K} \boldsymbol{J}(\boldsymbol{x}), & \boldsymbol{x} \in \Omega^{+},\end{cases}
$$

setting $\boldsymbol{J}=\boldsymbol{n} \times \boldsymbol{H}_{\mid \Gamma}^{\text {tot }}$ on $\Gamma$.

To derive suitable integral equations on $\Gamma$, the tangential traces of the potentials $\mathcal{T}$ and $\mathcal{K}$ have to be considered. To this end, let us formally introduce (see [100] for more rigorous definitions) the tangential gradient $\nabla_{\Gamma}$ and the tangential vector $\operatorname{curl} \operatorname{curl}_{\Gamma}$ which are linear continuous operators, as well as their respective adjoint operators $\operatorname{div}_{\Gamma}$ and $\operatorname{curl}_{\Gamma}$. The Laplace-Beltrami operator acting on a function $u$ is defined by

$$
\Delta_{\Gamma} u=\operatorname{div}_{\Gamma} \nabla_{\Gamma} u=-\operatorname{curl}_{\Gamma} \operatorname{curl}_{\Gamma} u,
$$

and the Hodge operator applying on a tangent vector field $\boldsymbol{v}$ is

$$
\boldsymbol{\Delta}_{\Gamma} \boldsymbol{v}=\nabla_{\Gamma} \operatorname{div}_{\Gamma} \boldsymbol{v}-\operatorname{curl}_{\Gamma} \operatorname{curl}_{\Gamma} \boldsymbol{v} .
$$

For a smooth vector function $\boldsymbol{v} \in \mathcal{C}^{\infty}(\bar{\Omega})$, let us introduce [99, 100] the tangential trace mapping $\gamma_{t}: \boldsymbol{v} \mapsto \boldsymbol{v}_{\mid \Gamma} \times \boldsymbol{n}$. We then have the following result 56 , 99.

Proposition 9 The exterior (+) and interior (-) tangential traces of the potentials $\mathcal{T}$ and $\mathcal{K}$ on $\Gamma$ are given by

$$
\begin{aligned}
& \left(\gamma_{t}^{ \pm} \circ \mathcal{T} \boldsymbol{J}\right)(\boldsymbol{x})=T \boldsymbol{J}(\boldsymbol{x}) \times \boldsymbol{n}(\boldsymbol{x}), \boldsymbol{x} \in \Gamma, \\
& \left(\gamma_{t}^{ \pm} \circ \mathcal{K} \boldsymbol{M}\right)(\boldsymbol{x})= \pm \frac{1}{2} \boldsymbol{M}(\boldsymbol{x})+K \boldsymbol{M}(\boldsymbol{x}) \times \boldsymbol{n}(\boldsymbol{x}), \boldsymbol{x} \in \Gamma,
\end{aligned}
$$

with, for $\boldsymbol{x} \in \Gamma$,

$$
\begin{aligned}
& T \boldsymbol{J}(\boldsymbol{x})=\int_{\Gamma} G(\boldsymbol{x}, \boldsymbol{y}) \boldsymbol{J}(\boldsymbol{y}) d \Gamma(\boldsymbol{y})+\frac{1}{k^{2}} \nabla_{\Gamma} \int_{\Gamma} G(\boldsymbol{x}, \boldsymbol{y}) \operatorname{div}_{\Gamma} \boldsymbol{J}(\boldsymbol{y}) d \Gamma(\boldsymbol{y}), \\
& K \boldsymbol{M}(\boldsymbol{x})=\int_{\Gamma} \nabla_{\boldsymbol{y}} G(\boldsymbol{x}, \boldsymbol{y}) \times \boldsymbol{M}(\boldsymbol{y}) d \Gamma(\boldsymbol{y}) .
\end{aligned}
$$


To avoid the problem of interior resonances, Brakhage and Werner 31] proposed the following combined potentials formulation

$$
\begin{cases}\boldsymbol{E}^{+}(\boldsymbol{x})=\mathcal{K} \boldsymbol{a}(\boldsymbol{x})-i \eta k Z_{0} \mathcal{T}(\boldsymbol{n} \times \boldsymbol{a})(\boldsymbol{x}), & \boldsymbol{x} \in \Omega^{+}, \\ \boldsymbol{H}^{+}(\boldsymbol{x})=\frac{1}{i k Z_{0}} \operatorname{curl} \boldsymbol{E}^{+}(\boldsymbol{x}), & \boldsymbol{x} \in \Omega^{+} .\end{cases}
$$

The coupling parameter $\eta$ is a real-valued number and $\boldsymbol{a}$ is a fictitious density. Taking the exterior tangential trace $\gamma_{t}^{+}$of the electric field 70 and for the boundary condition (63), we derive the following boundary integral equation

$$
\left(\frac{1}{2} \mathrm{I}-\boldsymbol{n} \times K\right) \boldsymbol{a}+i \eta k Z_{0}(\boldsymbol{n} \times T)(\boldsymbol{n} \times \boldsymbol{a})=-\boldsymbol{E}_{\mid \Gamma}^{\mathrm{inc}} \times \boldsymbol{n}, \text { on } \Gamma
$$

It can be proved [56] that the integral equation $(71)$ is well-posed for any wavenumber $k>0$ and parameter $\eta>0$ if $\Gamma$ is sufficiently smooth. In [87, Kress numerically shown that $\eta=1$ yields the lowest condition number for (71). However, despite this optimal choice, the combined integral equation (71) remains a first-kind integral equation.

Now, taking the tangential traces in 68

$$
\begin{cases}\boldsymbol{E}_{\mid \Gamma}^{+} \times \boldsymbol{n}=i k Z_{0} T \boldsymbol{J} \times \boldsymbol{n}, & \text { on } \Gamma, \\ \boldsymbol{H}_{\mid \Gamma}^{+} \times \boldsymbol{n}=-\frac{1}{2} \boldsymbol{J}+\boldsymbol{n} \times K \boldsymbol{J}, & \text { on } \Gamma,\end{cases}
$$

and imposing the perfectly conducting boundary condition 63 leads to the Electric Field Integral Equation (EFIE) and the Magnetic Field Integral Equation (MFIE), respectively given by

$$
i k Z_{0} T \boldsymbol{J}=-\boldsymbol{E}_{T}^{\mathrm{inc}} \text { and }\left(\frac{1}{2} \mathrm{I}+\boldsymbol{n} \times K\right) \boldsymbol{J}=\boldsymbol{n} \times \boldsymbol{H}_{\mid \Gamma}^{\mathrm{inc}}, \text { on } \Gamma
$$

with $\boldsymbol{E}_{T}^{\text {inc }}=\boldsymbol{n} \times\left(\boldsymbol{E}_{\mid \Gamma}^{\text {inc }} \times \boldsymbol{n}\right)$, and where I is the identity operator. These integral equations may be ill-posed for certain frequencies. To get a well-posed formulation, Harrington and Mautz 73. proposed the following Combined Field Integral Equation (CFIE)

$$
\mathrm{CFIE}=\alpha \mathrm{EFIE}+(1-\alpha) \mathrm{MFIE},
$$

where $\alpha \in] 0,1[$ is an arbitrary parameter. Even if the CFIE does not have any spurious mode, this well-posed integral equation is still a first-order compact perturbation of the EFIE. This means that, after discretization by a BEM, the resulting linear system remains badly conditioned, most particularly regarding the wavenumber $k$ and the mesh refinement. 
9.2 Generalized combined field integral equations

Similarly to the acoustic case, generalized CFIE can be derived to get a wellconditioned and well-posed integral equation to solve [58,59]. Here, for the sake of conciseness, we only present the main ideas without giving too much details.

Let us consider that we know the exact Electric-to-Magnetic operator $\Lambda^{\text {ex }}$ such that

$$
\boldsymbol{n} \times \boldsymbol{H}_{\mid \Gamma}^{+}+\Lambda^{\mathrm{ex}}\left(\boldsymbol{n} \times\left(\boldsymbol{E}_{\mid \Gamma}^{+} \times \boldsymbol{n}\right)\right)=\mathbf{0}, \quad \text { on } \Gamma,
$$

or equivalently

$$
\boldsymbol{J}+\Lambda^{\mathrm{ex}}(\boldsymbol{n} \times \boldsymbol{M})=\mathbf{0}, \quad \text { on } \Gamma .
$$

This operator can be introduced in a natural way in 65 , leading to

$$
\begin{cases}\boldsymbol{E}^{+}=\mathcal{K} \boldsymbol{M}-i k Z_{0} \mathcal{T} \Lambda^{\mathrm{ex}}(\boldsymbol{n} \times \boldsymbol{M}), & \text { in } \Omega^{+}, \\ \boldsymbol{H}^{+}=\frac{1}{i k Z_{0}} \operatorname{curl} \boldsymbol{E}^{+}, & \text {in } \Omega^{+} .\end{cases}
$$

Considering the tangential trace of the first equation of 74 , we obtain

$$
\left(\frac{1}{2} \mathrm{I}-\boldsymbol{n} \times K\right) \boldsymbol{M}+i k Z_{0}(\boldsymbol{n} \times T) \Lambda^{\mathrm{ex}}(\boldsymbol{n} \times \boldsymbol{M})=\boldsymbol{E}^{+} \times \boldsymbol{n}=\boldsymbol{M}, \text { on } \Gamma,
$$

and hence the following integral relation on $\Gamma$

$$
\left(\frac{1}{2} \mathrm{I}-\boldsymbol{n} \times K\right)+i k Z_{0}(\boldsymbol{n} \times T) \Lambda^{\mathrm{ex}} \circ \boldsymbol{n} \times=\mathrm{I},
$$

which corresponds to an ideal situation if one wants to solve the integral equation using a Krylov subspace iterative solver. However, the operator $\Lambda^{\text {ex }}$ is never known explicitly in practice for a general surface $\Gamma$. As for the acoustic case, the main idea is now to consider an approximation $\widetilde{\Lambda}$ of the exact operator $\Lambda^{\mathrm{ex}}$ and to represent the exterior electromagnetic field as

$$
\begin{cases}\boldsymbol{E}^{+}=\mathcal{K} \boldsymbol{a}-i k Z_{0} \mathcal{T} \widetilde{\Lambda}(\boldsymbol{n} \times \boldsymbol{a}), & \text { in } \Omega^{+}, \\ \boldsymbol{H}^{+}=\frac{1}{i k Z_{0}} \operatorname{curl} \boldsymbol{E}^{+}, & \text {in } \Omega^{+} .\end{cases}
$$

The corresponding combined integral equation is then

$$
\left(\frac{1}{2} \mathrm{I}-\boldsymbol{n} \times K\right) \boldsymbol{a}+i k Z_{0}(\boldsymbol{n} \times T) \widetilde{\Lambda}(\boldsymbol{n} \times \boldsymbol{a})=-\boldsymbol{E}_{\mid \Gamma}^{\mathrm{inc}} \times \boldsymbol{n}, \text { on } \Gamma,
$$

for the non-physical surface field $\boldsymbol{a}$. Let us remark that the choice of the Silver-Müller condition applied on the surface $\Gamma$, i.e. $\widetilde{\Lambda}=I$, leads to the standard BW integral formulation for the optimal coupling parameter $\eta=1$ proposed by Kress. Consequently, this approach can be interpreted as a natural generalization of the Brakhage-Werner integral formulation. 
In the same spirit, a generalized CFIE can be proposed. Applying first $\widetilde{\Lambda}$ to regularize the EFIE and next adding the MFIE yields

$$
\left(\frac{1}{2} I+\boldsymbol{n} \times K-i k Z_{0} \widetilde{\Lambda} T\right) \boldsymbol{J}=\boldsymbol{n} \times \boldsymbol{H}_{\mid \Gamma}^{\mathrm{inc}}+\widetilde{\Lambda} \boldsymbol{E}_{T}^{\mathrm{inc}}, \text { on } \Gamma
$$

Unlike $\boldsymbol{a}$ in (76), the surface current $\boldsymbol{J}$ is a physical unknown quantity, which makes this integral equation more attractive for applications. In practice, one possible suitable choice for $\tilde{\Lambda}$ is

$$
\widetilde{\Lambda}=Z_{0}^{-1}\left(\mathrm{I}+\frac{\boldsymbol{\Delta}_{\Gamma}}{k_{\delta}^{2}}\right)^{-1 / 2}\left(\mathrm{I}-\frac{1}{k_{\delta}^{2}} \operatorname{curl}_{\Gamma} \operatorname{curl}_{\Gamma}\right) .
$$

The optimal damping parameter $\delta$ is given by $\delta=0.6 k^{1 / 3} \mathcal{H}^{-2 / 3}$, where $\mathcal{H}$ is the local mean curvature of $\Gamma$. The operator $\widetilde{\Lambda}$ can be computed accurately and efficiently based again on the complex Padé approximants (58) and using adapted BEMs [64]. In addition, the generalized BW and CFIE are well-posed at any frequency [58, generalizing hence the standard case. Finally, the corresponding operators have an excellent clustering of their eigenvalues [58].

To illustrate this last property, we solve both the standard CFIE with optimal coupling parameter $(\alpha=0.2$ is usually used in practice for the linear combination), the generalized CFIE with nonlocal operator $\widetilde{\Lambda}$ and its localized version with Padé approximants for the unit sphere at $k=35$. The solution can be obtained in the basis of spherical harmonics (see [58] for more details). We use the GMRES iterative solver without restart and a tolerance equal to $10^{-6}$ on the reduction of the initial residual. We plot on Figure 19 the history of the residual versus the iteration number until convergence. We set the maximal number of Fourier modes for the series expansion to $3 k, Z_{0}=1$ and $\varepsilon=0.6 k^{1 / 3}$. The generalized CFIE (before and after localization) clearly leads to the best convergence rate, with a significant reduction of the number of iterations compared to the standard CFIE formulation. In fact, it can be shown that the eigenvalue clustering at a point close to $(1,0)$ explains this superlinear convergence. For more general scatterers, BEMs are needed, coupled to acceleration algorithms like the FMM or H-matrix approaches.

\section{Generalized Combined Field Integral Equations in elasticity}

In this section, we present some recent developments for the fast iterative solution of high-frequency scattering problems in elastodynamics using the integral equation method. First, we introduce the exterior Navier problem and the material to derive integral equations. Next, we give the main lines for the construction of well-conditioned CFIEs. We finally report some numerical simulations to illustrate their efficiency. 


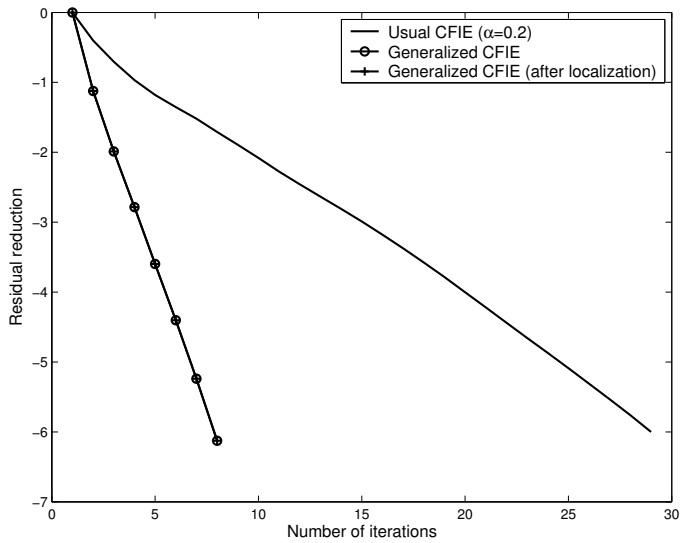

Fig. 19 History of the residual norm vs. the number of iterations for the unit sphere (for $k=35)$.

10.1 The exterior Navier problem and standard boundary integral equations

The propagation of time-harmonic waves in a three-dimensional isotropic and homogeneous elastic medium $\Omega^{+}$is governed by the Navier equation [85. Eq. (12.5) page 55] for an angular frequency $\omega>0$. The medium is characterized by the Lamé parameters $\mu_{L}$ and $\lambda_{L}$ and the density $\rho$ which are positive constants. The scattered field $\boldsymbol{u}^{+}$is decomposed into a longitudinal field $\boldsymbol{u}_{p}$ with vanishing curl and a transverse divergence-free field $\boldsymbol{u}_{s}$ solutions to

$$
\Delta \boldsymbol{u}_{p}+\kappa_{p}^{2} \boldsymbol{u}_{p}=0 \text { and curlcurl } \boldsymbol{u}_{s}-\kappa_{s}^{2} \boldsymbol{u}_{s}=0,
$$

with respective wavenumbers $\kappa_{p}^{2}=\rho \omega^{2}\left(\lambda_{L}+2 \mu_{L}\right)^{-1}$ and $\kappa_{s}^{2}=\rho \omega^{2} \mu_{L}^{-1}$. The vector Laplace operator is $\boldsymbol{\Delta}$. The behavior of the scattered displacement field $\boldsymbol{u}^{+}$at infinity is described by the Kupradze radiation conditions 85 , Eqs (2.6)-(2.9) page 126] uniformly in all directions. The scattering problem is formulated as follows : given an incident displacement wave $\boldsymbol{u}^{\text {inc }}$ which is assumed to solve the Navier equation in the absence of any scatterer, find the field $\boldsymbol{u}^{+}=\boldsymbol{u}_{p}+\boldsymbol{u}_{s}$, satisfying a Dirichlet boundary condition, solution to the boundary-value problem

$$
\left\{\begin{array}{l}
\mu_{L} \boldsymbol{\Delta} \boldsymbol{u}^{+}+\left(\lambda_{L}+\mu_{L}\right) \boldsymbol{\nabla} \operatorname{div} \boldsymbol{u}^{+}+\rho \omega^{2} \boldsymbol{u}^{+}=0, \text { in } \Omega^{+}, \\
\boldsymbol{u}^{+}=-\boldsymbol{u}^{\text {inc }}, \text { on } \Gamma, \\
\lim _{r \rightarrow \infty} r\left(\frac{\partial \boldsymbol{u}_{p}}{\partial r}-i \kappa_{p} \boldsymbol{u}_{p}\right)=0, \quad \lim _{r \rightarrow \infty} r\left(\frac{\partial \boldsymbol{u}_{s}}{\partial r}-i \kappa_{s} \boldsymbol{u}_{s}\right)=0, r=\|\boldsymbol{x}\| .
\end{array}\right.
$$

The fundamental solution of the Navier equation is a 3-by-3 matrix-valued function which is expressed by

$$
\begin{aligned}
\Phi(\boldsymbol{x}, \boldsymbol{y})=\frac{1}{\rho \omega^{2}}\left(\operatorname{curl}_{\operatorname{curl}}\left\{G\left(\kappa_{s}, \boldsymbol{x}-\boldsymbol{y}\right) \mathrm{I}_{3}\right\}\right. \\
\left.-\nabla_{\boldsymbol{x}} \operatorname{div}_{\boldsymbol{x}}\left\{G\left(\kappa_{p}, \boldsymbol{x}-\boldsymbol{y}\right) \mathrm{I}_{3}\right\}\right),
\end{aligned}
$$


with $G$ the free-space Green's function given by 18 . The single- and doublelayer potential operators are respectively defined by

$$
L \boldsymbol{\varphi}=\int_{\Gamma} \Phi(\cdot, \boldsymbol{y}) \boldsymbol{\varphi}(\boldsymbol{y}) d s(\boldsymbol{y}) \quad \text { and } \quad D \boldsymbol{\psi}=\int_{\Gamma}\left[\boldsymbol{T}_{\boldsymbol{y}} \Phi(\cdot, \boldsymbol{y})\right]^{\top} \boldsymbol{\psi}(\boldsymbol{y}) d s(\boldsymbol{y}),
$$

where $\boldsymbol{T}_{\boldsymbol{y}} \Phi(\boldsymbol{x}, \boldsymbol{y})$ is the tensor obtained by applying the traction operator $\boldsymbol{T}_{\boldsymbol{y}}=\boldsymbol{T}\left(\boldsymbol{n}(\boldsymbol{y}), \partial_{\boldsymbol{y}}\right)$ (see Eq. (84)) to each column of $\Phi(\boldsymbol{x}, \boldsymbol{y})$. The scattered field is expressed by the Somigliana integral representation [85, 86 .

$$
\boldsymbol{u}^{+}(\boldsymbol{x})=D \boldsymbol{u}_{\mid \Gamma}^{+}(\boldsymbol{x})-L \boldsymbol{t}_{\mid \Gamma}(\boldsymbol{x}), \quad \boldsymbol{x} \in \Omega^{+},
$$

where the Neumann trace, defined by $\boldsymbol{t}_{\mid \Gamma}:=\boldsymbol{T} \boldsymbol{u}$, is given through the traction operator

$$
\boldsymbol{T}=2 \mu_{L} \frac{\partial}{\partial \boldsymbol{n}}+\lambda_{L} \boldsymbol{n} \operatorname{div}+\mu_{L} \boldsymbol{n} \times \mathbf{c u r l} .
$$

By using the potential theory introduced in Section 4 , the standard CFIE reads: find $\boldsymbol{\psi}=-\left(\boldsymbol{t}_{\mid \Gamma}+\boldsymbol{t}_{\mid \Gamma}^{\text {inc }}\right) \in\left(H^{-1 / 2}(\Gamma)\right)^{3}$, with $\boldsymbol{t}_{\mid \Gamma}^{\text {inc }}=\boldsymbol{T} \boldsymbol{u}^{\text {inc }}$, solution to

$$
\left(\frac{\mathcal{I}}{2}+\mathcal{N}^{\prime}+i \alpha \mathcal{L}\right) \psi=-\left(\boldsymbol{t}_{\mid \Gamma}^{\mathrm{inc}}+i \alpha \boldsymbol{u}_{\mid \Gamma}^{\mathrm{inc}}\right), \quad \text { on } \Gamma
$$

with the following boundary integral operators

$$
\begin{aligned}
\mathcal{L} \boldsymbol{\varphi}(\boldsymbol{x}) & =\int_{\Gamma} \Phi(\boldsymbol{x}, \boldsymbol{y}) \boldsymbol{\varphi}(\boldsymbol{y}) d s(\boldsymbol{y}) \\
\mathcal{N}^{\prime} \boldsymbol{\varphi}(\boldsymbol{x}) & =\int_{\Gamma} \boldsymbol{T}_{\boldsymbol{x}}\{\Phi(\boldsymbol{x}, \boldsymbol{y}) \boldsymbol{\varphi}(\boldsymbol{y})\} d s(\boldsymbol{y})
\end{aligned}
$$

for a given vector density $\boldsymbol{\varphi}$ and $\boldsymbol{x} \in \Gamma$. The CFIE 85 is proved to be wellposed for any frequency $\omega>0$ and any non-zero real-valued parameter $\alpha$ [76, 85, 86. Recent works in the BEM community have been devoted to the derivation of fast evaluation techniques to perform the matrix-vector product needed in the iterative GMRES solver 45,46, leading to mature efficient algorithms. However, similarly to the acoustic and electromagnetic cases, the standard CFIE does not provide sufficiently good spectral properties at high frequencies, and hence it is not very well-suited for an iterative solver [48,61.

10.2 Generalized combined field integral equations

The construction of generalized CFIEs for the Navier equation is not a direct extension of the acoustic and electromagnetism cases. Some difficulties inherent to elasticity have to be addressed. Let us define the exact exterior Dirichlet-to-Neumann (DtN) map

$$
\boldsymbol{\Lambda}^{\mathrm{ex}}: \boldsymbol{u}_{\mid \Gamma}^{+} \in\left(H^{1 / 2}(\Gamma)\right)^{3} \mapsto \boldsymbol{t}_{\mid \Gamma}:=\boldsymbol{\Lambda}^{\mathrm{ex}} \boldsymbol{u}_{\mid \Gamma}^{+} \in\left(H^{-1 / 2}(\Gamma)\right)^{3} .
$$


Using the same approach described in acoustics, we get

$$
\frac{\mathrm{I}}{2}+\mathcal{N}^{\prime}-\boldsymbol{\Lambda}^{\mathrm{ex}^{\prime}} \mathcal{L}=\mathrm{I}, \quad \text { on } \Gamma,
$$

and the Calderón's relations lead to the integral representations of the adjoint DtN map

$$
\Lambda^{\mathrm{ex}^{\prime}}=-\left(\frac{1}{2} \mathrm{I}-\mathcal{N}^{\prime}\right) \mathcal{L}^{-1}=\left(\frac{1}{2} \mathrm{I}+\mathcal{N}^{\prime}\right)^{-1} \mathcal{S}
$$

with the hypersingular boundary integral operator $\mathcal{S}$. A good regularizing operator for $\mathcal{L}$, and hence for the CFIE, is an approximate adjoint DtN map $\tilde{\boldsymbol{\Lambda}}^{\prime}$. A particularity in elasticity is that the double-layer boundary integral operator $\mathcal{N}$ and its adjoint $\mathcal{N}^{\prime}$ are not compact even for sufficiently smooth boundaries. Their principal parts must also be considered in the approximation $\tilde{\boldsymbol{\Lambda}}^{\prime}$ (this is not the case in acoustics, see Section 8.1). Obtaining the expressions of the principal part of each elementary boundary integral operator is a difficult task. Recent works proposed approximations of various orders for the exact adjoint DtN map [47,61. To this end, a modified potential theory was applied where the tangential Günter derivative plays an important role. The approximations of the DtN and the NtD maps are expressed in terms of surface differential operators, square-root operators and their inverse. As an example, the principal part $P(\mathcal{N})$ of the operator $\mathcal{N}$ is decomposed as the sum of two terms, i.e. $P(\mathcal{N})=\mathcal{N}_{1}+\mathcal{N}_{2}$, given by

$$
\begin{aligned}
\mathcal{N}_{1}= & \frac{i}{2}\left(\boldsymbol{n}\left(\Delta_{\Gamma}+\kappa_{p}^{2} \mathrm{I}\right)^{-\frac{1}{2}} \operatorname{div}_{\Gamma} \mathrm{I}_{\boldsymbol{\tau}}-\boldsymbol{\nabla}_{\Gamma}\left(\Delta_{\Gamma}+\kappa_{s}^{2} \mathrm{I}\right)^{-\frac{1}{2}} \boldsymbol{n} \cdot \mathrm{I}_{\boldsymbol{n}}\right), \\
\mathcal{N}_{2}= & \frac{i \mu_{L}}{\rho \omega^{2}}\left(-\boldsymbol{n}\left(\Delta_{\Gamma}+\kappa_{s}^{2} \mathrm{I}\right)^{\frac{1}{2}} \operatorname{div}_{\Gamma} \mathrm{I}_{\boldsymbol{\tau}}+\boldsymbol{n} \Delta_{\Gamma}\left(\Delta_{\Gamma}+\kappa_{p}^{2} \mathrm{I}\right)^{-\frac{1}{2}} \operatorname{div}_{\Gamma} \mathrm{I}_{\boldsymbol{\tau}}\right. \\
& \left.\quad+\nabla_{\Gamma}\left(\Delta_{\Gamma}+\kappa_{p}^{2} \mathrm{I}\right)^{\frac{1}{2}}\left(\boldsymbol{n} \cdot \mathrm{I}_{\boldsymbol{n}}\right)-\boldsymbol{\nabla}_{\Gamma}\left(\Delta_{\Gamma}+\kappa_{s}^{2} \mathrm{I}\right)^{-\frac{1}{2}} \Delta_{\Gamma}\left(\boldsymbol{n} \cdot \mathrm{I}_{\boldsymbol{n}}\right)\right),
\end{aligned}
$$

where $\mathrm{I}_{\boldsymbol{n}}=\boldsymbol{n} \otimes \boldsymbol{n}$ ( $\otimes$ is the tensor of two vectors) and $\mathrm{I}_{\boldsymbol{\tau}}=\mathrm{I}-\mathrm{I}_{\boldsymbol{n}}$. The generalized CFIE then reads: find $\boldsymbol{\psi}=-\left(\boldsymbol{t}_{\mid \Gamma}+\boldsymbol{t}_{\mid \Gamma}^{\text {inc }}\right) \in\left(H^{-1 / 2}(\Gamma)\right)^{3}$ such that

$$
\left(\frac{\mathrm{I}}{2}+\mathcal{N}^{\prime}-\tilde{\boldsymbol{\Lambda}}^{\prime} \mathcal{L}\right) \boldsymbol{\psi}=-\left(\boldsymbol{t}_{\mid \Gamma}^{\mathrm{inc}}-\tilde{\boldsymbol{\Lambda}}^{\prime} \boldsymbol{u}_{\mid \Gamma}^{\mathrm{inc}}\right), \quad \text { on } \Gamma .
$$

We report in Tables 1 and 2 the number of iterations \#iter of the GMRES (with no restart and tolerance $10^{-3}$ ) for solving the standard CFIE (85) and three generalized CFIE (called P-CFIE) with approximations $\tilde{\boldsymbol{\Lambda}}^{\prime}$ of different orders $(\mathrm{LO}=$ low-order, $\mathrm{HO}=$ high-order), for increasing frequency $\omega$. Here again, complex Padé approximants are applied to get accurate local representations of the square-root operators and their inverses. These 3D results, which are obtained in [48, attest the drastic convergence acceleration of the generalized CFIE. 


\begin{tabular}{cccccc}
\hline \#DOFs & $\omega$ & $\begin{array}{c}\text { \# iter } \\
\text { CFIE }\end{array}$ & $\begin{array}{c}\text { \# iter LO } \\
\text { P-CFIE }\end{array}$ & $\begin{array}{c}\text { \# iter HO(1) } \\
\text { P-CFIE }\end{array}$ & $\begin{array}{c}\text { \# iter HO(2) } \\
\text { P-CFIE }\end{array}$ \\
\hline 1926 & 4 & 18 & 9 & 7 & $6(10)$ \\
7686 & 8.25 & 26 & 10 & 7 & $4(11)$ \\
30726 & 16.5 & 75 & 11 & 7 & $4(14)$ \\
122886 & 33 & 199 & 14 & 8 & $4(15)$ \\
490629 & 66.5 & $>500$ & 16 & 10 & $4(16)$ \\
\hline
\end{tabular}

Table 1 Diffraction of S-waves by the unit sphere. Number of GMRES iterations \# iter for a fixed density of 10 points per wavelength. The variable \#DOFs denotes the number of degrees of freedom.

\begin{tabular}{cccccc}
\hline \#DOFs & $\omega$ & $\begin{array}{c}\text { \# iter } \\
\text { CFIE }(\eta=1)\end{array}$ & $\begin{array}{c}\text { \# iter LO } \\
\text { P-CFIE }\end{array}$ & $\begin{array}{c}\text { \# iter HO }(1) \\
\text { P-CFIE }\end{array}$ & $\begin{array}{c}\text { \# iter HO }(2) \\
\text { P-CFIE }\end{array}$ \\
\hline 1446 & 2.5 & 14 & 10 & 9 & $9(13)$ \\
6630 & 5 & 40 & 12 & 10 & $9(13)$ \\
26505 & 11 & 120 & 13 & 10 & $9(12)$ \\
105990 & 22 & $>500$ & 14 & 11 & $9(13)$ \\
\hline
\end{tabular}

Table 2 Diffraction of P-waves by a cube. Number of GMRES iterations \# iter for a fixed density of 10 points per wavelength. The variable \#DOFs denotes the number of degrees of freedom.

\section{Additional contributions and references}

We end this paper by referencing some related contributions for preconditioning surface integral equations in acoustics, electromagnetism and elasticity. There is an extensive literature on this subject. The following list of references is arbitrary and not exhaustive. Additional references should be of course cited for completeness. We select some of the representative works which should help as a guideline for further reading, but we restrict the list for the sake of conciseness.

In acoustics, more developments concerning the Calderón preconditioning are available e.g. in 68. Initially, closely related approaches to analytical preconditioning and generalized CFIE were obtained by Levadoux in his Ph.D. Thesis [92. Generalized CFIE for the sound-hard case with corners was considered in 20. The case of an impedance boundary condition with Lipschitz boundary was analyzed in [115. Regularized high-order integral equations solvers were derived in 33] for 3D acoustic problems. This kind of preconditioning was also studied for the iterative solution of acoustic [27,28,30 and electromagnetic 27. transmission problems. The situation of heterogeneous media is treated in 44 based on a weak FEM-BEM coupling between using optimized domain decomposition techniques. This leads to similar issues as for the generalized CFIE. Let us also mention an alternative and promising approach, called multi-trace formulation, for solving multi-subdomain scattering problems. It has been introduced in 74 and improved in a series of contributions (e.g [54 104]). Finally, a successful practical application of the generalized CFIE derived in Section 8 was proposed in [117] to simulate high- 
intensity focused ultrasound techniques used for the non-invasive treatment of cancer.

In electromagnetism, contributions to the Calderón preconditioning introduced by Christiansen and Nédélec 52,53, for the EFIE are given e.g. in 2, 3, 7, 8, 21, 67, 83, 97, 109. Domain decomposition preconditioners were recently proposed in 103,104 for the EFIE. Other classes of closely related generalized CFIE in electromagnetism were derived in $4,25,29,32,93,94$. Finally, more algebraic preconditioning techniques are also available in [41.

The Calderón preconditioner was studied in 77 for elastodynamics. Much less works are devoted to the construction of regularized CFIE for the Navier equation. For example, in 37, the authors derived a new regularized CFIE for 3D elastic scattering problems and the approach introduced in Section 10 is extended in [49] to the case of the Neumann boundary condition.

\section{Conclusion}

The aim of this paper was to introduce some recent achievements in the preconditioning of surface integral equations for acoustic, electromagnetic and elastic scattering problems. In particular, we discussed the high frequency regime which is a very active area where much work still remains to do. A selected review of some advances in the analytical preconditioning of surface integral equations as well as the construction of generalized CFIE has been detailed. Different points of view can be adopted like for example the integral or pseudodifferential approaches. Even in acoustics, many open questions still need to be further investigated and can impact similar situations like e.g. in electromagnetism or elasticity, where the numerical solution by boundary integral equations is also extremely challenging.

Other future exciting directions of research include e.g. the understanding of the effect of non convexity and geometrical singularities on an iterative solver, the coupling of analytical and algebraic preconditioners, the application of pseudodifferential operator theory to the design of hybrid analyticalalgebraic preconditioners for the finite element solution of time-harmonic waves, and of course the application to large scale industrial problems within fast integral solvers.

\section{References}

1. M. Abramowitz and I.A. Stegun, Handbook of Mathematical Functions, Dover, New York, (1974).

2. S.B. Adrian, F.P. Andriulli and T.F. Eibert, A hierarchical preconditioner for the electric field integral equation on unstructured meshes based on primal and dual Haar bases, J. Comput. Phys., 330, (2017), pp.365-379.

3. S.B. Adrian, F.P. Andriulli and T.F. Eibert, On a refinement-free Calderon multiplicative preconditioner for the electric field integral equation, J. Comput. Phys., 376, (2019), pp.1232-1252.

4. F. Alouges, S. Borel and D. Levadoux, A stable well-conditioned integral equation for electromagnetism scattering, J. Comput. Appl. Math. 204 (2) (2007), pp.440-451. 
5. S. Amini, On the choice of the coupling parameter in boundary integral formulations of the exterior acoustic problem, Appl. Anal. 35 (1-4), (1990), pp.75-92

6. S. Amini and S.M. Kirkup, Solution of Helmholtz equation in exterior domain by elementary boundary integral equations, J. Comput. Phys. 118 (1995), pp. 208-221.

7. F.P. Andriulli, K. Cools, K. Bagci, F. Olyslager, A. Buffa, S. Christiansen and E. Michielssen, A multiplicative Calderon preconditioner for the electric field integral equation, IEEE Transactions on Antennas and Propagation, 56(8,1), (2008), pp.2398-2412.

8. F.P. Andriulli, K. Cools, I. Bogaert and E. Michielssen, On a well-conditioned Electric Field Integral operator for multiply connected geometries, IEEE Transactions on Antennas and Propagation, 61(4,2), (2013), pp.2077-2087.

9. X. Antoine, Conditions de Radiation sur le Bord, Ph.D. Thesis, University of Pau, 1997.

10. X. Antoine, Fast approximate computation of a time-harmonic scattered field using the On-Surface Radiation Condition method, IMA J. Appl. Math. 66 (2001), pp.83-110.

11. X. Antoine, Advances in the On-Surface Radiation Condition Method: Theory, Numerics and Applications, Book Chapter in Computational Methods for Acoustics Problems, Editor F. Magoulès, Saxe-Coburg Publications, 2008, pp.169-194 (ISBN: 978-1-874672-30$2)$.

12. X. Antoine, H. Barucq and A. Bendali, Bayliss-Turkel-like radiation condition on surfaces of arbitrary shape, J. Math. Anal. Appl. 229 (1999), pp.184-211.

13. X. Antoine, A. Bendali and M. Darbas, Analytic preconditioners for the Electric Field Integral Equation, Int. J. Numer. Meth. Eng. 61 (2004), pp.1310-1331.

14. X. Antoine, A. Bendali and M. Darbas, Analytic preconditioners for the boundary integral solution of the scattering of acoustic waves by open surfaces, J. Comput. Acoust. 13 (3), (2005), pp.477-498.

15. X. Antoine and Y. Boubendir, An integral preconditioner for solving the twodimensional scattering transmission problem using integral equations, Int. J. Comput. Math., 85 (10), (2008), pp.1473-1490.

16. X. Antoine and M. Darbas, Alternative integral equations for the iterative solution of acoustic scattering problems, Quarterly J. Mech. Appl. Math. 58 (2005), pp. 107-128.

17. X. Antoine and M. Darbas, Generalized combined field integral equations for the iterative solution of the three-dimensional Helmholtz equation, Math. Model. Numer. Anal., 41 (1), (2007), pp.147-167.

18. X. Antoine, M. Darbas and Y.Y. Lu, An improved surface radiation condition for highfrequency acoustics scattering problems, Comput. Meth. Appl. Mech. Eng. 195 (33-36) (2006), pp.4060-4074.

19. X. Antoine, C. Geuzaine and K. Ramdani, Computational Methods for Multiple Scattering at High Frequency with Applications to Periodic Structures Calculations, M. Ehrhardt (Ed.), Wave Propagation in Periodic Media - Analysis, Numerical Techniques and Practical Applications, Progress in Computational Physics, Vol. 1, Bentham Science Publishers Ltd., 2009.

20. A. Arnand, J.S. Ovall and C. Turc, Well-conditioned boundary integral equations for two-dimensional sound-hard scattering problems in domains with corners, Journal of Integral Equations and Applications, 24(3), (2012), pp.321-358.

21. H. Bagci, F.P. Andriulli, K. Cools, F. Olyslager and E. Michielssen, A Calderon multiplicative preconditioner for the Combined Field Integral Equation, IEEE Transactions on Antennas and Propagation, 57(10) (2009), pp.3387-3392.

22. A. Bayliss, C.I. Goldstein and E. Turkel, An iterative method for the Helmholtz equation, J. Comput. Phys., 49, (1983), pp.443-457.

23. M. Benzi, Preconditioning techniques for large linear systems: A survey, J. Comput. Phys. 182(1), (2002), p.418-477.

24. M. Bollhoefer, M.J. Grote and O. Schenk, Algebraic multilevel preconditioner for the Helmholtz equation in heterogeneous media, SIAM J. Sc. Comput. 31 (5), (2009), pp.37813805 .

25. S. Borel, D.P. Levadoux and F. Alouges, A new well-conditioned integral formulation for Maxwell equations in three dimensions, IEEE Transactions on Antennas and Propagation 53(9) (2005), pp.2995-3004.

26. S. Börm, L. Grasedyck, W. Hackbusch, Introduction to hierarchical matrices with applications, Engineering Analysis with Boundary Elements, 27(5), (2003), pp.405-422. 
27. Y. Boubendir, O.P. Bruno, D. Levadoux and C. Turc, Integral equations requiring small numbers of Krylov-subspace iterations for two-dimensional smooth penetrable scattering problems, Applied Numerical Mathematics, 95(SI), (2015), pp.82-98.

28. Y. Boubendir, V. Dominguez, D. Levadoux and C. Turc, Regularized combined field integral equations for acoustic transmission problems, SIAM Journal on Applied Mathematics, 75(3), (2015), pp.929-952.

29. Y. Boubendir and C. Turc, Well-conditioned boundary integral equation formulations for the solution of high-frequency electromagnetic scattering problems, Computers and Mathematics with Applications, 67(10), (2014), pp.1772-1805.

30. Y. Boubendir, C. Turc and V. Dominguez, High-order Nystrom discretizations for the solution of integral equation formulations of two-dimensional Helmholtz transmission problems, IMA Journal of Numerical Analysis, 36 (1), (2016), pp.463-492.

31. H. Brakhage and P. Werner, Über das Dirichletsche Aussenraumproblem für die Helmholtzsche Schwingungsgleichung, Numer. Math., 16, (1965), pp.325-329.

32. O.P. Bruno, T. Elling, R. Paffenroth and C. Turc, Electromagnetic integral equations requiring small numbers of Krylov-subspace iterations, Journal of Computational Physics, 228 (17), (2009), pp.6169-6183.

33. O.P. Bruno, T. Elling and C. Turc, Regularized integral equations and fast high-order solvers for sound-hard acoustic scattering problems, International Journal for Numerical Methods in Engineering, 91 (10), (2012), pp.1045-1072.

34. O.P. Bruno and C. Geuzaine, An $\mathcal{O}(1)$ integration scheme for three-dimensional surface scattering problems, J. Comput. Appl. Math., 204, (2007), pp.463-476.

35. O. Bruno, C. Geuzaine, J. Monro Jr and F. Reitich, Prescribed error tolerances within fixed computational times for scattering problems of arbitrarily high frequency: the convex case, Philosophical Transactions of the Royal Society (Series A: Mathematical, Physical and Engineering Sciences), 362 (1816), (2004), pp.629-645.

36. O.P. Bruno and L.A. Kunyansky, High-order algorithm for the solution of surface scattering problems: Basic implementation, tests, and applications, J. Comput. Phys. 169, (2001), pp.80-110.

37. O.P. Bruno and T. Yin, Regularized integral equation methods for elastic scattering problems in three dimensions, J. Comput. Phys., 410, (2020), 109350.

38. A. Buffa and R. Hiptmair, Regularized combined field integral equations, Numer. Math., 100 (1), (2005), pp.1-19.

39. A. Buffa and S.A. Sauter, On the acoustic single layer potential: stabilization and Fourier analysis, SIAM J. Sci. Comput. 28 (2006), pp.1974-1999.

40. A.J. Burton and G.F. Miller, The application of integral equation methods to the numerical solution of some exterior boundary-value problems, Proc. Roy. Soc. London. Ser. A, 323, (1971), pp.201-210.

41. B. Carpinteri, Preconditioning for large-scale boundary integral equations in electromagnetics, IEEE Transactions on Antennas and Propagation, 56(6), (2014), pp.338-345.

42. B. Carpentieri, I. S. Duff, and L. Giraud, Sparse pattern selection strategies for robust Frobenius-norm minimization preconditioners in electromagnetics, Numer. Linear Algebra Appl. 7 (2000), pp. 667-685.

43. B. Carpentieri, I. S. Duff and L. Giraud, Experiments with sparse approximate preconditioning of dense linear problems form electromagnetic applications, Tech. Rep. TR/PA/00/04, CERFACS, France, (2000).

44. B. Caudron, X. Antoine and C. Geuzaine, Optimized weak coupling of boundary element and finite element methods for acoustic scattering, J. Comp. Phys. 421, (2020), 109737.

45. S. Chaillat and M. Bonnet, Recent advances on the fast multipole accelerated boundary element method for 3D time-harmonic elastodynamics, Wave Motion, 50 (2013), pp.10901104.

46. S. Chaillat, M. Bonnet and J.F. Semblat, A multi-level fast multipole BEM for 3-D elastodynamics in the frequency domain, Comput. Methods Appl. Mech. Engng., 197 (2008), pp.4233-4249.

47. S. Chaillat, M. Darbas and F. Le Louër, Approximate local Dirichlet-to-Neumann map for three-dimensional time-harmonic elastic waves, Computer Methods in Applied Mechanics and Engineering, 297 (2015), pp.62-83. 
48. S. Chaillat, M. Darbas and F. Le Louër, Fast iterative boundary element methods for high-frequency scattering problems in 3D elastodynamics, J. Comput. Phys. 341 (2017), pp.429-446.

49. S. Chaillat, M. Darbas and F. Le Louër, Analytical preconditioners for Neumann elastodynamic Boundary Element Methods, under revision.

50. S.N. Chandler-Wilde, I.G. Graham, S. Langdon and M. Lindner, Condition number estimates for combined potential boundary integral operators in acoustic scattering, J. Int. Eq. Appl. 21 (2) (2009), pp.229-279.

51. W.C. Chew, J.M. Jin, E. Michielssen and J. Song, "Fast and Efficient Algorithms in Computational Electromagnetics", Artech House Antennas and Propagation Library, Norwood, 2001.

52. S.H. Christiansen, Résolution des Equations Intégrales pour la Diffraction d'Ondes Acoustiques et Electromagnétiques. Stabilisation d'Algorithmes Itératifs et Aspects de l'Analyse Numérique, Ph.D. Thesis, Ecole Polytechnique, Palaiseau, France, (2001).

53. S.H. Christiansen and J.C. Nédélec, A preconditioner for the electric field integral equation based on Calderon formulas, SIAM Journal on Numerical Analysis, 40(3), (2002), pp.1100-1135.

54. X. Clayes and R. Hiptmair, Multi-trace boundary integral formulation for acoustic scattering by composite structures, Communications on Pure and Applied Mathematics,66(8), (2013), pp.1163-1201.

55. R. Coifman, V. Rokhlin and S. Wandzura, The Fast Multipole Method for the wave equation: A pedestrian description, IEEE Trans. on Ant. and Prop., 35(3), (1993), pp.712.

56. D.L. Colton and R. Kress, Integral Equation Methods in Scattering Theory, Pure and Applied Mathematics, John Wiley and Sons Inc., 1983.

57. M. Costabel, Integral equation methods in scattering theory, SIAM J. Math. Anal. 19, (1988), pp.613-626.

58. M. Darbas, Préconditionneurs Analytiques de Type Calderon pour les Formulations Intégrales des Problèmes de Diffraction d'Ondes, Ph.D. Thesis, Toulouse, 2004.

59. M. Darbas, Generalized combined field integral equations for the iterative solution of the three-dimensional Maxwell equations, Applied Mathematics Letters, 19 (8), (2006), pp.834-839.

60. M. Darbas, E. Darrigrand, and Y. Lafranche, Combining OSRC preconditioning and Fast Multipole Method for the Helmholtz equation, J. Comput. Phys., 236 (2013), pp.289316 .

61. M. Darbas and F. Le Louër, Well-conditioned boundary integral formulations for the iterative solution of elastic scattering problems, Mathematical Methods in the Applied Sciences, 38 (2015), pp.1705-1733.

62. E. Darrigrand, Couplage Méthodes Multipôles Rapides et Discrétisation Microlocale pour les Equations Intégrales de l'Electromagnétisme, Ph.D. Thesis, Bordeaux, 2002.

63. E. Darve, Méthodes Multipôles Rapides: Résolution des Equations de Maxwell par Formulations Intégrales, Ph.D. Thesis, Paris 6, 1999.

64. M. El Bouajaji, X. Antoine, and C. Geuzaine, Approximate local Magnetic-to-Electric surface operators for time-harmonic Maxwell's equations, J. Comput. Phys., 279 (15) (2014), pp. 241-260.

65. S. Engleder and O. Steinbach, Stabilized boundary element methods for exterior Helmholtz problems, Numer. Math. 110 (2008), pp.145-160.

66. Y. A. Erlangga, C. Vuik and C.W. Oosterlee, On a class of preconditioners for the Helmholtz equation, Appl. Numer. Math., 50, (2004), pp.409-425.

67. P. Escapil-Inchauspe and C. Jerez-Hanckes, Fast Calderon preconditioning for the Electric Field Integral Equation, IEEE Transactions on Antennas and Propagation, 67 (4,2), (2019), pp.2555-2564.

68. I. Fierro and C. Jerez-Hanckes, Fast Calderon preconditioning for Helmholtz boundary integral equations, J. Comp. Phys., 409, (2020), 109355.

69. A. Greenbaum, Iterative Methods for Solving Linear Systems, Frontiers in Applied Mathematics, 27, Society for Industrial and Applied Mathematics (SIAM), Philadelphia, PA, 1997.

70. L. Greengard and V. Rokhlin, A fast algorithm for particle simulations, J. Comput. Phys., 73 (2), (1987), pp.325-348. 
71. M.J. Grote and T. Huckle, Parallel preconditioning with sparse approximate inverses, SIAM J. Sc. Comput. 18 (3), (1997), pp.838-853.

72. W. Hackbusch, Hierarchical Matrices: Algorithms and Analysis, Vol. 49 of Springer Series in Computational Mathematics, Springer, Heidelberg,2015.

73. R.F. Harrington and J.R. Mautz, H-field, E-field and combined field solution for conducting bodies of revolution, Arch. Elektron. Übertragungstech (AEÜ), 32 (4) (1978) 157-164.

74. R. Hiptmair and C. Jerez-Hanckes, Multiple traces boundary integral formulation for Helmholtz transmission problems, Advances in Computational Mathematics, 37(1), (2012), pp.39-91.

75. P.L. Ho and Y.Y. Lu, Improving the beam propagation method for TM polarization, Opt. Quantum Electronics, 35(4), (2003), pp. 507-519.

76. G.C. Hsiao and W.L. Wendland, Boundary Integral Equations, Vol. 164 of Applied Mathematical Sciences, Springer-Verlag, Berlin, 2008.

77. H. Isakari, K. Niino, H. Yoshikawa, and N. Nishimura, Calderon's preconditioning for periodic fast multipole method for elastodynamics in 3D, International Journal for Numerical Methods in Engineering, 90(4), (2012), pp.484-505.

78. D.S. Jones, An approximate boundary condition in acoustics, J. Sound Vibr. 121 (1) (1988), pp. 37-45.

79. R. Kerchroud, A. Soulaimani and X. Antoine, Performance study of plane wave finite element methods with a Padé-type artificial boundary condition in acoustic scattering, Adv. Eng. Soft. 40 (2009), pp. 738-750.

80. R. Kerchroud, A. Soulaimani and Y. Saad, Preconditioning techniques for the solution of the Helmholtz equation by the finite element method, Math. Comput. Simul., 65 (4-5), (2004), pp.303-321.

81. B. Kim and J.W. Kang, A time-domain formulation of elastic waves in heterogeneous unbounded domains, Multiscale Science and Engineering, 1, (2019), pp.220-235.

82. S. Kirkup, The Boundary Element Method in acoustics: a survey, Applied SciencesBasel, 9(8), (2019), DOI: 10.3390/app9081642.

83. A. Kleanthous, T. Betcke, D.P. Hewett, M.W. Scroggs and A.J. Baran, Calderon preconditioning of PMCHWT boundary integral equations for scattering by multiple absorbing dielectric particles, Journal of Quantitative Spectroscopy and Radiative Transfer, 224, (2019), pp.383-395.

84. F. Kpadonou, S. Chaillat and P. Ciarlet, On the efficiency of nested GMRES preconditioners for $3 D$ acoustic and elastodynamic H-matrix accelerated Boundary Element Methods, Computers and Mathematics with Applications, 80(3), (2020), pp.471-489.

85. V.D. Kupradze, Potential methods in the theory of elasticity, Translated from the Russian by H. Gutfreund. Translation edited by I. Meroz, Israel Program for Scientific Translations, Jerusalem, 1965.

86. V.D. Kupradze, T.G. Gegelia, M.O. Basheleŭshvilii, and T.V. Burchuladze, Threedimensional problems of the mathematical theory of elasticity and thermoelasticity, Vol. 25 of North- Holland Series in Applied Mathematics and Mechanics, North-Holland Publishing Co., Amsterdam, russian ed., 1979. Edited by V.D. Kupradze.

87. R. Kress, Minimizing the condition number of boundary integral operators in acoustic and electromagnetic scattering, Quart. J. Mech. Appl. Math., 38 (2), (1985), pp.323-341.

88. R. Kress, Linear Integral Equations, Second Ed., Applied Mathematical Sciences (82), New York, Springer-Verlag, 1999.

89. R. Kress and W.T. Spassov, On the condition number of boundary integral operators for the exterior Dirichlet problem for the Helmholtz equation, Numer. Math., 42 (1), (1983), pp.77-95.

90. G.A. Kriegsmann, A. Taflove and K.R. Umashankar, A new formulation of electromagnetic wave scattering using the on-surface radiation condition method, IEEE Trans Antennas Propag. 35 (1987), pp. 153-161.

91. S. Lang, Linear Algebra, Springer, 3rd edition, 2004.

92. D.P. Levadoux, Etude d'une Equation Intégrale Adaptée à la Résolution Hautes Fréquences de l'Equation de Helmholtz, Ph.D. Thesis, Université Paris VI, 2001.

93. D.P. Levadoux, Stable integral equations for the iterative solution of electromagnetic scattering problems, Comptes Rendus Physique, 7(5) (2006), pp.518-532. 
94. D.P. Levadoux, Some preconditioners for the CFIE equation of electromagnetism, Mathematical Methods in the Applied Sciences, 31(17), (2008), pp.2015-2028.

95. W. McLean, Strongly Elliptic Systems and Boundary Integral Equations, Cambridge University Press, Cambridge, 2000.

96. J. Liesen and P. Tichy, Convergence analysis of Krylov subspace methods, GAMMMitteilungen, 27(2), (2004), pp. 153-173.

97. Y. Mao, J. Niu, Q. Zhan, R. Zhang, W. Huang, and Q.H. Liu, Calderon preconditioned spectral-element spectral-integral method for doubly periodic structures in layered media, IEEE Transactions on Antennas and Propagation, 68(7), (2020), pp.5524-5533.

98. F.A. Milinazzo, C.A. Zala and G.H. Brooke, Rational square-root approximations for parabolic equation algorithms, J. Acoust. Soc. Am. 101 (2), (1997), pp. 760-766.

99. P. Monk, Finite Element Methods for Maxwell's Equations, Oxford University Press, New York, 2003

100. J.-C. Nédélec, Acoustic and Electromagnetic Equations, Applied Mathematical Sciences (144), New York, Springer-Verlag, 2001.

101. D. Osei-Kuffuor and Y. Saad, Preconditioning Helmholtz linear systems, Applied Numerical Mathematics 60(4), (2010), pp.420-431.

102. Z. Peng, A novel multitrace boundary integral equation formulation for electromagnetic cavity scattering problems, IEEE Transactions on antennas and Propagation, 63(10), (2015), pp.4446-4457.

103. Z. Peng, R. Hiptmair, Y. Shao, and B. MacKie-Mason, Domain decomposition preconditioning for surface integral equations in solving challenging electromagnetic scattering problems, IEEE Transactions on Antennas and Propagation, 64(1), (2016), pp. 210-223.

104. Z.Peng, X.-C. Wang and J.-F. Lee, Integral equation based domain decomposition method for solving electromagnetic wave scattering from non-penetrable objects, IEEE Transactions on Antennas and Propagation, 59(9), (2011), pp.3328-3338.

105. V. Rokhlin, Rapid solution of integral equations of scattering theory in two dimensions, J. Comput. Phys. 86 (2) (1990), pp. 414-439.

106. Y. Saad, Iterative Methods for Sparse Linear Systems", PWS Publishing Company, Boston, 1996.

107. Y. Saad and M.H. Schultz, A generalized minimal residual algorithm for solving nonsymmetric linear systems, SIAM J. Sci. Comput., 7 (3), (1986), pp.856-869.

108. L. Schwartz, Théorie des Distributions, Broché, 1997.

109. M.W. Scroggs, T. Betcke, E. Burman, W. Smigaj, and E. van't Wout, Software frameworks for integral equations in electromagnetic scattering based on Calderon identities, Computers and Mathematics with Applications, 74(11), (2017), pp.2897-2914.

110. O. Steinbach and W. L. Wendland, The construction of some efficient preconditioners in the boundary element method, Adv. Comput. Math. 9 (1-2) (1998), pp. 191-216.

111. G. Sylvand, La Méthode Multipôle Rapide en Electromagnétisme: Performances, Parallélisation, Applications, Thèse de Doctorat, ENPC, 2002.

112. M.E. Taylor, Pseudodifferential Operators, Princeton Mathematical Series, 34, Princeton University Press, 1981, xi +452 .

113. L.L. Thompson, A review of finite-element methods for time-harmonic acoustics, J. Acoust. Soc. Amer., 119 (3), (2006), pp.2272-2293.

114. L.N. Trefethen, Pseudospectra of Matrices, editors D.F. Griffiths, G.A. Watson., in "Numerical Analysis", Longman Scientific and Technical, 1991.

115. C. Turc, Y. Boubendir and M. Riahi, Well-conditioned boundary integral equation formulations and Nystrom discretizations for the solution of Helmholtz problems with impedance boundary conditions in two-dimensional lipschitz domains, Journal of Integral Equations and Applications, 29(3), (2017), pp.441-472.

116. E. Turkel, Boundary Conditions and Iterative Schemes for the Helmholtz Equation in Unbounded Regions, Book Chapter in Computational Methods for Acoustics Problems, Editor F. Magoulès, Saxe-Coburg Publications, 2008, pp. 127-159 (ISBN: 978-1-874672$30-2)$.

117. E. van't Wout, P. Gelat, T. Betcke and S. Arridge, A fast boundary element method for the scattering analysis of high-intensity focused ultrasound, Journal of the Acoustical Society of America, 138(5), (2015), pp.2726-2737.

118. Y. Wang, J. Lee and J. Zhang, A short survey on preconditioning techniques for largescale dense complex linear systems in electromagnetics, Int. J. Comp. Math. 84 (8) (2007), pp. 1211-1223. 